\title{
Influence of Heterogeneities on Waves of Excitation in the Heart
}

\author{
Dissertation \\ for the award of the degree \\ "Doctor rerum naturalium" \\ of the Georg-August-Universität Göttingen \\ within the doctoral program GGNB \\ of the Georg-August University School of Science (GAUSS) \\ submitted by \\ Tariq Baig-Meininghaus \\ from Hagen
}

Göttingen, 2017 


\section{Thesis Committee}

Prof. Dr. Stefan Luther, Research Group Biomedical Physics, Max Planck Institute for Dynamics and Self-Organization

Prof. Dr. Stefan Klumpp, Institut für Nichtlineare Dynamik, Georg-August-Universität Göttingen

Prof. Dr. Tim Friede, Institut für Medizinische Statistik, Georg-August-Universität Göttingen

Prof. Dr. Wolfram Kollatschny, Institut für Astrophysik, Georg-August-Universität Göttingen

Members of the Examination Board

Referee: Prof. Dr. Stefan Luther

$2^{\text {nd }}$ Referee: Prof. Dr. Stefan Klumpp

Further members of the Examination Board:

Prof. Dr. Tim Friede, Institut für Medizinische Statistik, Georg-August-Universität Göttingen

Prof. Dr. Wolfram Kollatschny, Institut für Astrophysik, Georg-August-Universität Göttingen

Dr. Karen Alim, Max Planck Institute for Dynamics and Self-Organization

Date of oral examination: 7.9.2017 


\section{Affidavit}

Hereby, I declare that the presented thesis has been written independently and with no other sources and aids than quoted.

Göttingen, 07.07.2017 


\section{Contents}

1. Introduction 1

1.1. Structure and Organization . . . . . . . . . . . . . 2

I. General 3

2. Anatomy 5

2.1. The Heart . . . . . . . . . . . . . . . . . . . . . . . 5

2.2. Coronary Vasculature . . . . . . . . . . . . . . . . 5

2.3. Cardiac Tissue . . . . . . . . . . . . . . . 6

2.3.1. Cardiomyocytes .................... 6

2.3.2. Gap junctions . . . . . . . . . . . . . . . . 7

2.3.3. Action Potential . . . . . . . . . . . . . 8

2.3.4. Electrical Stimulation . . . . . . . . . . . . . . 8

2.4. Collective behavior . . . . . . . . . . . . . . . . . . . 8

2.4.1. The heart beat ...................... 8

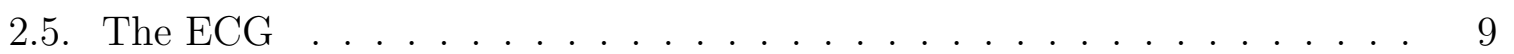

3. The Heart as Excitable Medium 11

3.1. Models for Cardiac Dynamics . . . . . . . . . . . . . . . . . . 11

3.1.1. Excitable Media . . . . . . . . . . . . . . . . . . 11

3.1.2. The Bidomain Model . . . . . . . . . . . . . . . . 13

3.2. Spiral waves . . . . . . . . . . . . . . . . . . . . . . . . . . . . . . . . . . . . .

3.3. Vulnerability . . . . . . . . . . . . . . . . . 17

3.4. Spiral Pinning . . . . . . . . . . . . . . . . . . . . . . . . . . 19

3.5. Spiral Unpinning . . . . . . . . . . . . . . . . . . . . . . . . . . . 21

3.6. Unpinning by Multiple Shocks . . . . . . . . . . . . . . . . . . . . 23

3.6.1. The Phase Transition Curve of a Pinned Spiral . . . . . . . . . 24

3.6.2. An Iterated Map for Multiple Shocks . . . . . . . . . . . . . . . 27

4. Arrhythmias 31

4.1. VF: Ventricular Fibrillation . . . . . . . . . . . . . . . . . . 31

4.2. VT: Ventricular Tachycardia . . . . . . . . . . . . . . . . . 32

4.3. Cardioversion . . . . . . . . . . . . . . . . . . 33

4.3.1. Determinism in Cardioversion . . . . . . . . . . . . 34 
4.3.2. Mechanisms of Defibrillation . . . . . . . . . . . . 34

5. Models of Myocardial Infarction 37

5.1. Myocardial Infarction . . . . . . . . . . . . . . . . . . . . 37

5.2. Models of Chronic Myocardial Infarction . . . . . . . . . . . . . 38

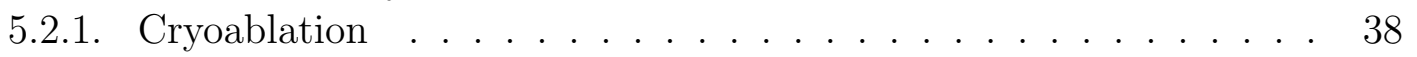

5.2 .2$. Ischemia . . . . . . . . . . . . . . . . . . . 39

6. Methods 41

6.1. Delay Reconstruction . . . . . . . . . . . . . . . . . . . . 41

6.2. Shannon Jensen Metric . . . . . . . . . . . . . . . . . . 43

6.3. PCA on Video Data . . . . . . . . . . . . . . . . . . 45

6.4. Spearman Correlation . . . . . . . . . . . . . . . . 48

6.5. Mantel Test . . . . . . . . . . . . . . . . . . . . . . . . . . 49

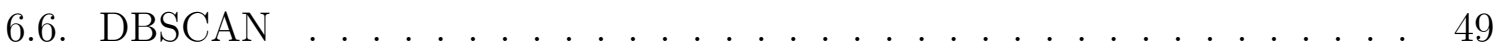

II. Ventricular Tachycardia 51

7. Rationale and Aims 53

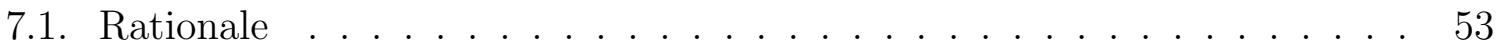

7.2. Aims . . . . . . . . . . . . . . . . . 54

8. Experimental Methods 55

8.1. Euthanasia and Heart Preparation . . . . . . . . . . . . . . 56

8.1.1. Pig ...................... 56

8.1.2. Rabbit ..................... . . . 56

8.2. Perfusion and Data Acquisition . . . . . . . . . . . 57

8.3. Electrodes . . . . . . . . . . . . . . . . . . . . 58

8.3.1. Configurations for Pigs . . . . . . . . . . . 59

8.3.2. Configuration for Rabbits . . . . . . . . . . . . . . 59

8.4. Injury Induction . . . . . . . . . . . . . . . . . . . . . . . 60

8.4.1. Cryoablation ................... 60 60

8.4.2. Ischemia in Rabbits . . . . . . . . . . . . . . . . . . . 60 60

8.4.3. Ischemia in Pigs . . . . . . . . . . . . . . . . . 61 61

8.4.4. Myocardial Infarction . . . . . . . . . . . . . . . . . 6 61

8.5. VT Induction . . . . . . . . . . . . . . . . . . . . 61

8.6. Measurement protocols . . . . . . . . . . . . . . . 62 62

8.7. Criteria to End the Experiment . . . . . . . . . . . . . 63

8.8. Drugs . . . . . . . . . . . . . . . . . . . . . 64 64

8.9. Details for the experiments . . . . . . . . . . . 64

9. Experimental Results 67

9.1. Overview ........................ . . . . . . . . . . 
9.2. Grouping VT . . . . . . . . . . . . . . . . . . . . . . . . . 69 69

9.3. Distance between VT episodes . . . . . . . . . . . . . . . . . . . . . . 70

9.3.1. Delay Reconstruction from the ECG . . . . . . . . . . . . . 70

9.3.2. Histogramming . . . . . . . . . . . . . . . . . . . . . 71

9.3.3. Measuring Similarity . . . . . . . . . . . . . . . 71

9.3.4. Clustering . . . . . . . . . . . . . . . . 73

9.3.5. Similarity Measure Evaluation . . . . . . . . . . . . . . . 73

9.4. Analysis . . . . . . . . . . . . . . . . . . . 78

9.4.1. Stability of the VT Morphologies under Shock . . . . . . . . . 78

9.4.2. Reinduceability . . . . . . . . . . . . . . 81

9.5. Model Comparison . . . . . . . . . . . . . . . . . 866

9.5.1. Cryoablation in Rabbits . . . . . . . . . . . . . . 87

9.5.2. Ischemia . . . . . . . . . . . . . . . . . . . . . 91

9.5.3. Myocardial Infarction . . . . . . . . . . . . . . . . . 97

9.5.4. Conclusion . . . . . . . . . . . . . . . . . . 103

9.6. Continued Analysis of 2016-06-21 . . . . . . . . . . . . . . . . 104

9.6.1. Phase Dependence and Phase Transition . . . . . . . . . . . . . . 105

9.6.2. Discussion of the Phase Transition Curve . . . . . . . . . . . . . 109

10.Summary and Outlook 117

10.1. Summary . . . . . . . . . . . . . . . . . . . . . . . . . 117

10.1.1. Distance Measure . . . . . . . . . . . . . . . . . . . . . 117

10.1.2. Animal Models . . . . . . . . . . . . . . . . . . . . 118

10.1.3. Phase Transition . . . . . . . . . . . . . . . . . . . 118

10.2. Outlook . . . . . . . . . . . . . . . . . . . . 118 


\section{Abbreviations}

ATP Antitachycardia Pacing

PTC Phase Transition Curve

VF Ventricular Fibrillation

VT Ventricular Tachycardia 


\section{Dedication}

To my wife Svenja, my son Darius and all the people I love. 


\section{Introduction}

Few people remember accurately the last time they sneezed, even though sneezing is an interruption of the normal respiratory function of the body. Those that have suffered "skipped heart beats" ${ }^{1}$ on the other hand tend to be keenly aware of the phenomenon ([98]), although it is in most cases just as dangerous as sneezing ([13]). The reason for this is that the heart is an extremely reliable organ, for a healthy adult it beats on average 80 times per minute, 4,800 times per day. In a normal life time the heart is estimated to beat more than 3 billion times, and during all this time it must never fail for more than a few seconds. As a consequence of this high reliability we are not used to even the briefest interruptions of the normal cardiac cycle.

Whenever the normal function of the heart is more severely disturbed the consequences are dire: reduced pumping power will result in shortness of breath and dizziness, total loss of pumping will result in a collapse of the circulatory system. $20 \%$ of all deaths in Europe are due to so called sudden cardiac death (SCD) ([1]), the sudden and unexpected total loss of cardiac function.

The origins of SCD lie in the unique way the muscle contractions that drive the hearts pumping are moderated: Cardiomyocytes form an excitable contracting syncytium. Each cell is capable of undergoing a reversible change in transmembrane potential, thereby activating its neighbors to do the same. It is this activation that causes the cells to contract, and the normal heart beat that originates at specialized pacemaker cells therefore spreads across the heart like a Mexican wave ${ }^{2}$,

During sudden cardiac death this wave spread gets disturbed. Local symmetry breaking leads to the formation of disorganized reoccurring patterns on the cardiac muscle. The heart loses its rhythm, it becomes arrhythmic, rather than beating it starts to quiver and in the most severe cases ceases to do its work. The only reliable treatment known so far for these desynchronization states is the application of high-energy electrical shock to the heart that resets all activity.

Heterogeneities such as blood vessels interact with the excitation waves on the heart, and there is the long standing hypothesis that arrhythmias are stabilized by their pres-

\footnotetext{
${ }^{1}$ So called palpitations

${ }^{2}$ Better known as La-Ola wave to Europeans.
} 


\section{Introduction}

ences $([133])$. It is hence of no surprise that survivors of myocardial infarction, whose myocardium is scarred, have an especially high risk of SCD (84]). To reduce mortality in these patients, it is advised by current clinical guidelines ([140]) that they be implanted with an internal defibrillator. Although this reduces the overall mortality this procedure is still a trade-off. While the number of deaths due to arrhythmias is reduced by an implanted defibrillator, at the same time the number of patients that experience pathological remodeling of the cardiac muscle is increased ([83], [93]).

Recently new defibrillation methods have been proposed that could greatly reduce the required field strength and consequently avoid myocardial injuries that might further tissue remodeling ([74], [49], 6]). These methods rely on the property of cardiac tissue heterogeneities to act as virtual electrodes in the presence of electric fields. To optimize them a better understanding of the interactions between electrical fields, heterogeneities and arrhythmias is needed, as currently no comprehensive theory exists.

The objective of this work is to study these interactions, and as often in biological research the first step is the search for a model system. As studying interactions means studying changes in a systems state in response to a perturbation, in this case a model that allows for the repeated preparation of similar arrhythmias is needed. However, the notion of similarity itself will need clarification and it has to be made precise what preparing the same arrhythmia twice actually means. In a second step the response of the arrhythmic dynamics to weak perturbations by virtual electrodes can then be systematically assessed.

\subsection{Structure and Organization}

This document is split into two parts. Part $\mathbf{I}$ contains the necessary background information. First the anatomy and physiology of the heart are introduced in chapter 2. The collective behavior of cardiac tissue is then discussed from the viewpoint of non linear dynamics in chapter 3. Arrhythmias, disturbances of the normal cardiac rhythm are introduced in chapter 4 and a connection between the previous two chapters is shown. Finally animal models of arrhythmias are discussed in chapter 5 and the methods needed to analyze the performed experiments are introduced in chapter 6 .

Part II investigates ventricular tachycardia in infarction models. This investigation is motivated in chapter 7. The used experimental procedures and methods are then introduced in chapter 8. In chapter 9 a method to objectively measure the distance between different arrhythmia episodes is developed. The performed experiments are then analyzed using this method, and the different infarction models are compared. Finally elements of chapter 3 are revisited and some of the experimental results are discussed using methods of non linear dynamics. 
Part I.

General 



\section{Anatomy}

\subsection{The Heart}

The mammalian heart is a pulsed muscular pump located under the breast bone. It consists of four chambers which can be grouped into two functional units, called the right and the left side of the heart. Each side has two chambers: A thin walled atrium and a thick walled ventricle, and each side drives the blood flow in one of the two major blood circuits of the mammalian body.

From the venae cavae oxygen poor blood enters the right atrium, out of which it is pumped via the tricusp valve into the right ventricle and onwards via the pulmonary semilunar valve into the pulmonary arteries and finally the lungs. Blood which was oxygenated in the lungs reenters the heart via the vena pulmonalis into the left atrium, and therefrom is pumped through mitral valve into the ventricle which in turn pumps it through the aortic semilunar valve into the aorta. The reader is encouraged to compare words written in cursive to figure 2.1.

The four valves of the heart are located in one plane, called cardiac valvular plane and are joined by connective tissue, the cardiac skeleton, which is stiff compared to the surrounding muscle. Apart from anchoring the valves, the cardiac skeleton serves as the attachment point for the atrial and ventricular muscle, insulates the different compartments electrically against each other and stabilizes the overall structure.

\subsection{Coronary Vasculature}

Due to its high metabolic demand the heart has a specialized vascular system attached to it that spans across all scales of the heart, down almost to cell level. This tree like structure of arteries and veins is called the coronary vasculature. In all mammals it originates from two arteries that fork of the aorta directly above the semilunar valve: the left and right coronary artery.

Contrary to the general heart structure, which is well comparable between different 


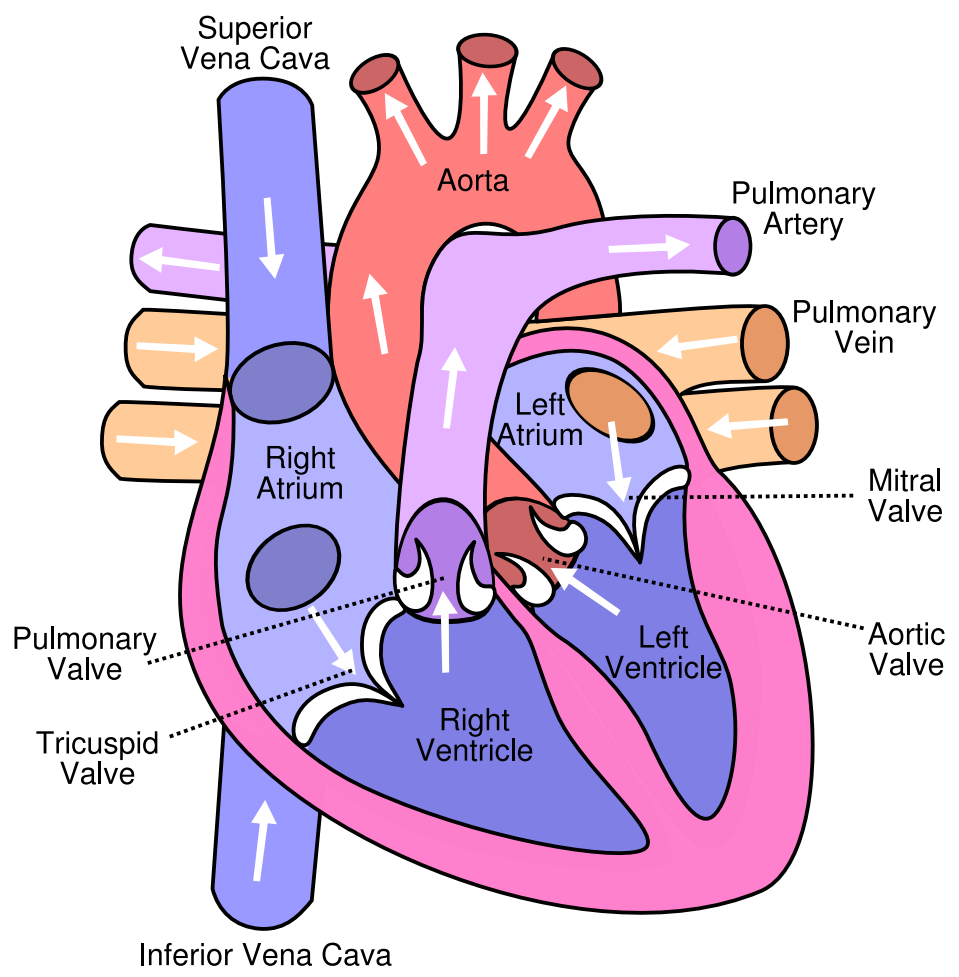

Figure 2.1.: Phases of the Action Potential - Schematic cross section of a human heart. Figure taken from [4].

species, the branching and distribution structure of the coronaries varies greatly ([50]): In humans and pigs the heart is typically provided for predominantly by the right coronary branch ([68]), while dogs typically have a left dominance and coronary structure of rabbits varies on an animal to animal basis ([92]). Another major point of difference between species is the presence of collateral perfusion, meaning the presence of tissue that is perfused by multiple coronary arteries. This is almost absent in humans and pigs, but clearly visible in dogs ([50]).

An overview of the human coronary arteries is shown in figure 2.2 .

\subsection{Cardiac Tissue}

\subsubsection{Cardiomyocytes}

Cardiomyocytes or heart muscle cells account for the active features of cardiac tissue: The ability to contract in response to and sustain electrical excitation. They make up roughly $70 \%$ of the total tissue volume. 


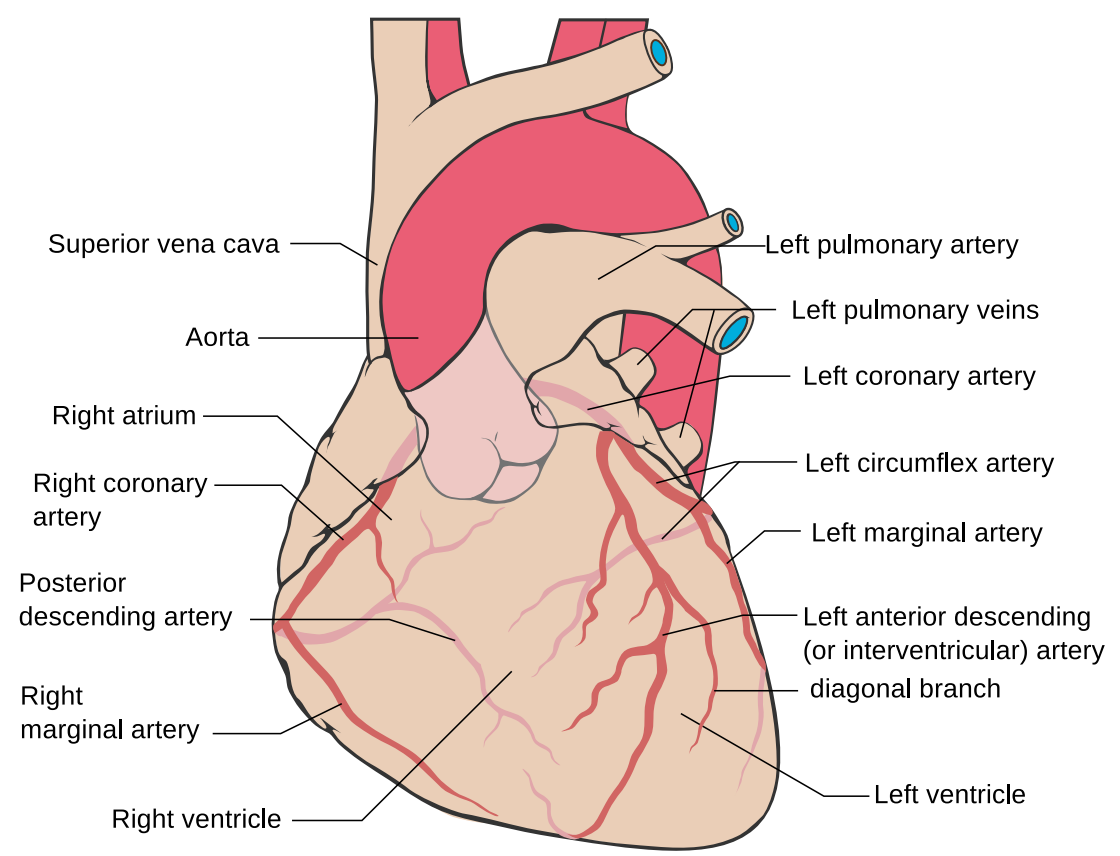

Figure 2.2.: Human coronary arteries - Schematic overview of the coronary arteries of the human heart, Anterior view. Figure taken from [3].

On the micro scale myocytes in mammals can be approximated reasonably well by elongated cylinders, although some branching may occur. These cylindrical shapes are coupled mechanically at the ends by specialized entities called intercalated disks resulting in a preferred direction of force transduction in direction of the long axis. Cells align locally parallel to this axis and form fibers, which are surrounded by a collagen network. Globally the fiber directions are not parallel, but twist in a complicated way.

\subsubsection{Gap junctions}

Electrical coupling is provided by gap junctions, which connect the intra cellular spaces of bordering cells creating a functional syncytium. Junctions can be found all over the cell wall but the majority is located at the intercalated disks leading to a better conduction in fiber direction.

As a result of ischemia (the local lack of nutrients and oxygen in tissue) or injury, gap junctions can cease to work, a process which is called gap junction uncoupling (see [48] and [124]). This effectively insulates the uncoupled cells from each other, greatly reducing the capability of the action potential to spread between them. 


\subsubsection{Action Potential}

In their quiescent state cardiac muscle cells possess a potential difference between cell interior and its surrounding. Each of these myocytes is capable of undergoing a reversible change in membrane potential consisting of depolarization and repolarization of the membrane capacitor, due to the opening and closing of several ion channels and the resulting ion exchange with the surrounding liquid. The overall voltage dynamics may be modeled as an excitable relaxation oscillator of Fitzhug-Nagumo type and is called action potential.

As a consequence of the gap junction coupling, ions can spread from a cell into its neighbors thus enabling collective behavior. In analogy to reaction diffusion systems this is called a wave of excitation.

In response to the local ion dynamics caused by an action potential a muscle cell contracts, and the visible pumping motion of the heart is a direct consequence of the action potential traveling over the heart muscle.

\subsubsection{Electrical Stimulation}

The ion channels that drive the action potential react to changes in the voltage across the membrane, hence the cell will be influenced by a forced change in trans membrane potential. Depolarizing the cell membrane across a threshold value by a cathodal stimulation will cause a new action potential, if the cell already is depolarized it may prolong an existing one. Hyperpolarization caused by an anodal stimulus on the other hand will suppress an occurring action potential or shorten the recovery time, a phenomenon which is called deexcitation. Additionally, on the tissue scale the velocity of excitation spread increases depending on the degree of Hyperpolarization, an effect that has been attributed to an temporal increase of available depolarizing ion channels [102].

\subsection{Collective behavior}

\subsubsection{The heart beat}

Since the cardiac skeleton insulates the different parts of the heart electrically against each other, the activation that originates at pacemaker cells in the right atrium cannot spread directly into the ventricles or the left atrium . A subsystem distributes the excitation via specialized fibers to the left atrium to the tip of the heart into the ventricles. 
Incorporated into this distribution system is a delay mechanism and as result of this delay the atria contract prior to the ventricles, which leads to the well known heart beat.

\subsection{The ECG}

The same shift in ion concentration that causes the membrane potential of a cell to change during an action potential gives rise to local electrical dipoles at the wave front. To see this consider, two cells, one of which is already depolarized and one of which is not. Since the membranes act as capacitors in both cases, the accumulated charges on the membranes differ between the two cells resulting in a dipole.

Integrating this local dipoles results in a net potential on the body surface. Measurements of this potential using electrodes are called electrocardiogram (ECG), and provide a non invasive method to obtain information about the dynamics on the heart muscle. 



\section{The Heart as Excitable Medium}

\subsection{Models for Cardiac Dynamics}

\subsubsection{Excitable Media}

It has been suggested in the late 60s that the electrical waves that orchestrate the complex mechanical behavior of the heart muscle can be modeled by equations of the following type:

$$
\partial_{t} R=\varphi(R)+\nabla D \nabla R
$$

for certain choices of $\varphi$ (see [138] for historical details), and by now the usefulness of this approach is widely accepted among researchers in the field of cardiac electrophysiology.

To be specific: $\varphi$ is chosen in such a way that it is an excitable oscillator of the FitzhugNagumo type, leading to a so called excitable medium ([11]). This corresponds to a continuum's limit of a medium built out of localized excitable entities, which are in this case cardiac cells ([85]). High dimensional, detailed ionic models of the cardiac action potential exist ([131]) and many features of the action potential can be reproduced in silico $([41])$.

As the subject of this document is not the simulation of cardiac tissue but its experimental investigation, here the simplest possible excitable medium shall be introduced: A cellular automaton possessing three states and three possible transitions. It will be used later on to illustrate some of the features of excitable media.

\section{States}

1. Excitable

2. Excited

3. Refractory 


\section{Transitions}
1. Excitable $\Rightarrow$ Excited
2. Excited $\Rightarrow$ Refractory
3. Refractory $\Rightarrow$ Excitable

Local coupling in this model is not provided by diffusion, but by the additional rule that excitable cells that have an excited neighbor become excited. The three states can be loosely identified with the different parts of the action potential (see figure 3.1) and are together with the specified rules already sufficient to allow for traveling waves (see figure 3.2 and spiral waves (to be introduced later).

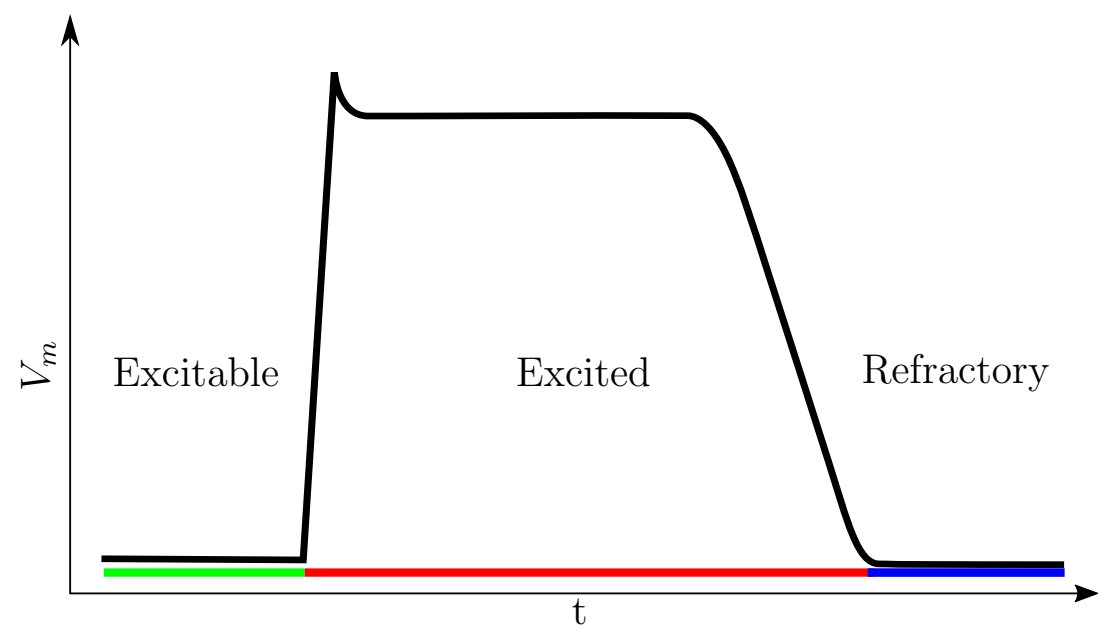

Figure 3.1.: Phases of the Action Potential - Schematic time course of the membrane potential of a single ventricular myocyte. The three different states of the simple cellular automaton can be associated to the action potential, although such a mapping is necessarily a simplification.

Excitable (green): During the excitable phase the cell will become excited if a sufficient number of its neighbors are.

Excited (red): During the excited phase the cell can excite other cells.

Refractory (blue): During the refractory phase the cell cannot be excited again by other cells. 


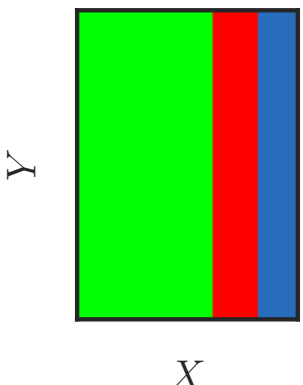

(a) $t=0$

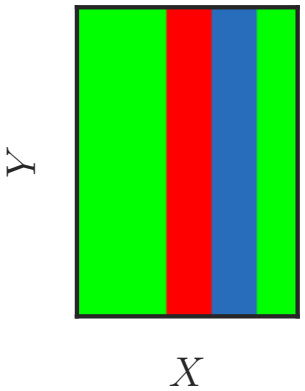

(b) $\mathrm{t}=1$

Figure 3.2.: Plane Wave in a Simple Cellular Automaton - States are colored as in figure 3.1. The wave is entering the domain from the right and traveling to the left. Points that are in front of the wave in $a$ at $\mathrm{t}=0$ become excited in $b$ at $\mathrm{t}=1$ while excited points become refractory.

\subsubsection{The Bidomain Model}

Although reaction diffusion systems can reproduce many qualitative features of the excitation spread in cardiac tissue, they lack the ability to correctly predict the tissue's response to an applied electrical field or injected current. To reproduce the observed de- and hyperpolarization of the cell membrane under this conditions, more complicated models were developed in the 1970s ([107]). Since these models use two separate domains called intra and extra cellular space, that occupy the same physical space they are called bidomain models.

In the purely passive bidomain model both spaces are viewed as networks of resistors and connected at every point by a capacitor in parallel with a resistor (see figure 3.3). When a field is applied, current flows in both spaces! however blood vessels, fiber curvature or injuries lead to a local increase in intra cellular resistivity which causes a current redistribution between the intra- and the extra cellular space. This redistribution in combination with the capacitive properties of the coupling between the spaces creates polarized areas near the conduction inhomogeneity ([102]).

In view of the fact that these areas act as wave emitting sites in the same way local electrodes do, they are called virtual cathodes. The hyperpolarized zones are accordingly referred to as virtual anodes (see figure 3.4 for an illustration of the redistribution of the currents).

Stronger fields create larger, more pronounced polarization zones. Similarly polarized

\footnotetext{
${ }^{1}$ Most models assume the spaces to have different conductivities.
} 
areas and degree of polarization are larger near bigger heterogeneities ([36]). As the membrane potential must be pushed across stimulation threshold for heterogeneities to act as wave sources for low field strength only the biggest of them are recruited as wave emitting sites.

The previously mentioned reaction diffusion systems can be recovered as special cases from bidomain models equipped with a more realistic local dynamics, assuming certain boundary conditions ([107]). Depending on the problem at hand reasoning from the reaction diffusion point of view or the bidomain point of view can be advantageous.

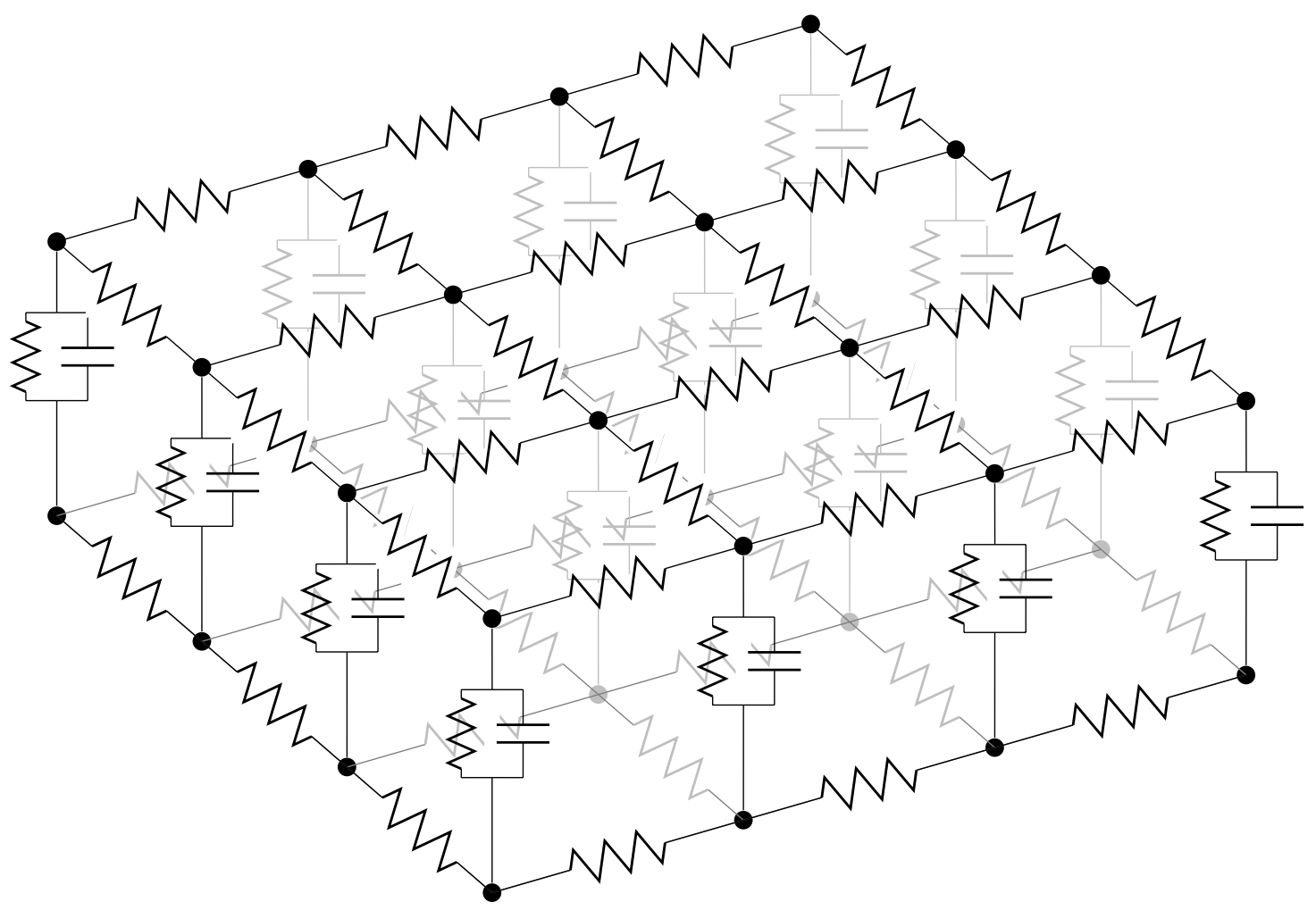

Figure 3.3.: Schematic representation of the bidomain model - Intra cellular space (above) and extra cellular space (below) occupy the same physical space and are interconnected by capacitor and a resistor in parallel. Both spaces are viewed as a network of resistors, the connecting RC-units represent the cell membranes of myocardial cells. If an electric field is applied, current flows freely in both of the domains and distributes according to their respective conductivities. 


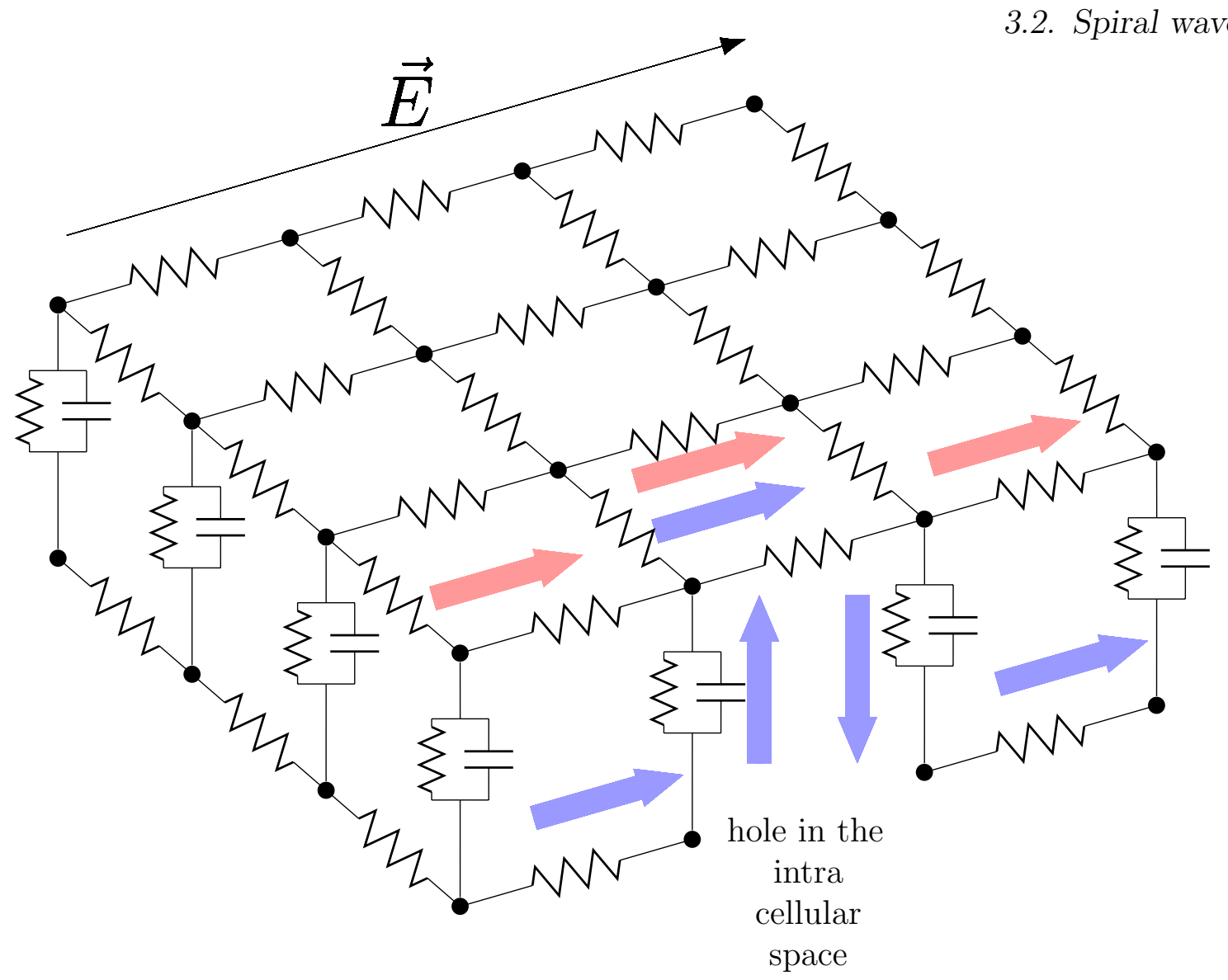

Figure 3.4.: Holes in the intra cellular space - Current flows in the extra cellular space (red) and the intra cellular space (blue) in response to an applied electrical field.

In presence of a hole or an area of greatly reduced conductivity in the intra cellular space, the intra cellular current redistributes into the extra cellular space. This causes the capacitors in the RC-units along its path to charge, resulting in areas of de- and hyperpolarization on opposing sites of the hole. Current injected into the spaces acts in a similar way, even in the absence of heterogeneities.

In a more complicated model including local dynamics the depolarized areas could then act as nucleation sites for waves of excitation.

\subsection{Spiral waves}

One striking feature of reaction diffusion systems are spiral waves. These spiral shaped, reentrant, spatiotemporal patterns form as a result of symmetry breaking due to inhomogeneity in the excitability of a system and persist even if this inhomogeneity is removed afterwards ([81]). Spiral waves, also called vortices or rotors, have been observed in such diverse systems as star clusters ([70]), chemical reactions ([137]), fungal growth patterns 


\section{The Heart as Excitable Medium}

([132]) and cardiac arrhythmias $([66])$.

In the heart spiral wave formation has been reported among others due to mechanical stimulation ([71]), faulty automaticity ([8]) and dynamical arising instabilities ([42]). Furthermore spiral waves were successfully related to tachycardia ([37]). Multiple mechanisms for the transition between stable rotating spirals and turbulent, chaotic behavior have been proposed. This so called spiral wave breakup is thought to underlie the often observed transformation from tolerable ventricular tachycardia to life threatening ventricular fibrillation ([42]).

An example of spiral wave formation in the presence of a temporary conduction block is shown in 3.5 for the simple automaton defined before; more dynamical spiral formation mechanisms are beyond this simple model.

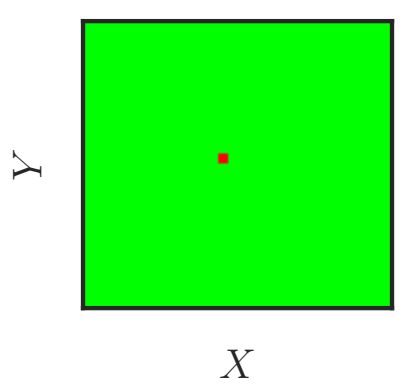

(a) $\mathrm{t}=0$

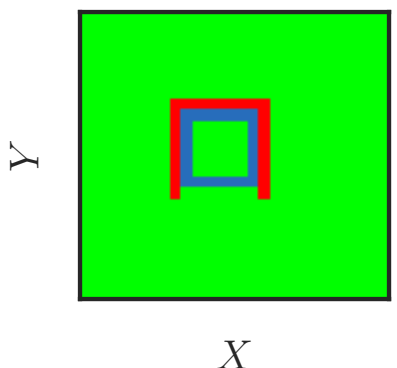

(d) $\mathrm{t}=4$

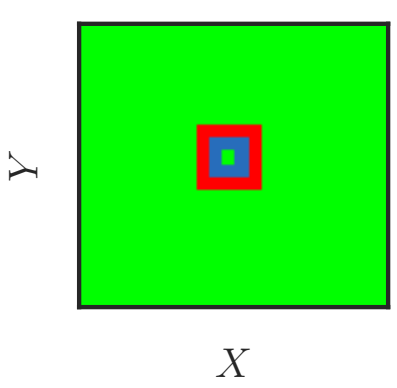

(b) $\mathrm{t}=2$

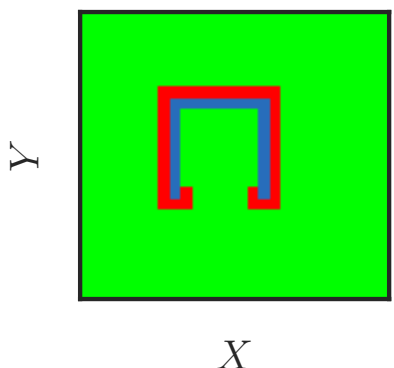

(e) $\mathrm{t}=5$

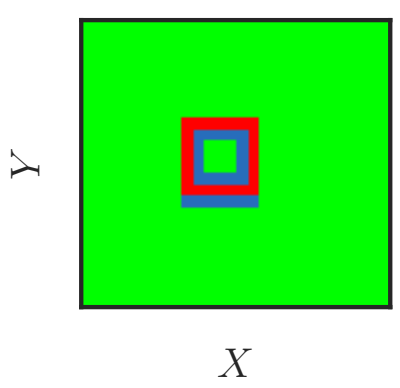

(c) $t=3$

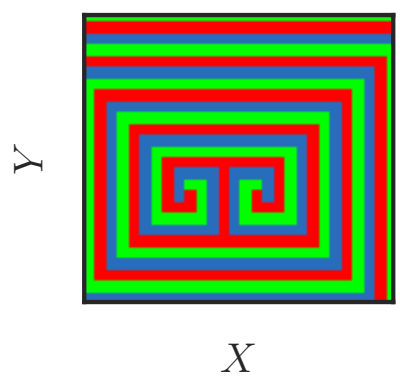

(f) $t=20$

Figure 3.5.: Spiral Wave in a Simple Cellular Automaton - States are colored as in figure 3.1. Time is in arbitrary units. A stimulus is applied $(\mathrm{t}=0)$ and nucleates into a ring $(t=2)$. Later a refractory barrier is placed in front of the ring $(t=3)$, which leads to the formation of an open $\operatorname{ring}(t=4)$. The sides of this open structure curve inwards $(t=5)$ and finally form a double spiral $(t=20)$ which is then stable.

In the absence of a stabilizing factor spiral waves in reaction diffusion systems can be 
pushed into the domain boundaries via an periodic stimulus that has higher frequency than the rotating spiral (see figure 3.6). This process is called antitachycardia pacing (ATP) in the cardiac sciences and part of the standard repertoire to treat cardiac arrhythmias ([23]).

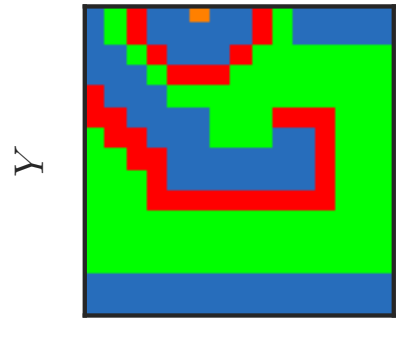

$X$

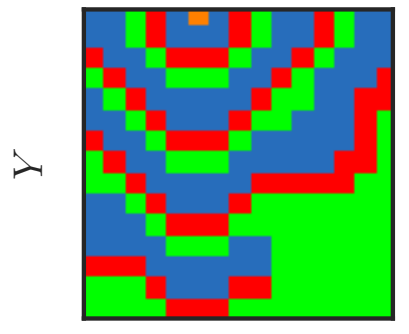

$X$

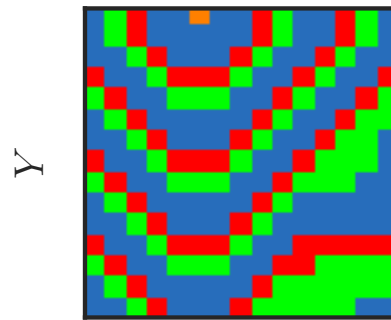

$X$

(a) $\mathrm{t}=0$

(b) $t=20$

(c) $\mathrm{t}=30$

Figure 3.6.: Spiral Termination by Overdrive Pacing - A spiral wave in a modified version of the earlier defined cellular automaton. States are colored as in figure 3.1 and a periodically pacing electrode was introduced at the upper border of the system (shown in orange). At first the spiral is freely rotating $(t=0)$. The waves emanating from the electrode push it towards the boundary $(t=20)$ until it vanishes $(t=30)$.

\subsection{Vulnerability}

The loose association between different parts of the action potential and excitability shown in figure 3.1 holds only for excitation spreading among cells. For external stimuli, such as an electrode injecting current or a virtual electrode, the situation is more complicated. In these cases it depends on the stimulus strength whether another action potential can be triggered or not (see figure 3.7).

For spatially extended electrodes this feature gives rise to a phenomenon called vulnerability ([118]): If in one dimensional medium a stimulus is applied over a region which is traversed by a wave of excitation, one part of that region might undergo an action potential while the other does not. The result of this is a second wave that travels in the opposite direction (see figure 3.8). The part of the spatial action potential that is susceptible to this broken front propagation is called the vulnerable window.

For higher dimensional media the situation is slightly different and a stimulus applied in the tail of a wave will form a double headed spiral (see figure 3.9 ). This has severe implications in case of the heart: As the normal heart beat spreads over the muscle in 
form of a plane wave, a pacemaker or implanted defibrillator that triggers at the wrong time might create a reentrant spiral wave in the muscle, which via spiral breakup can initiate fibrillation $([20])$.

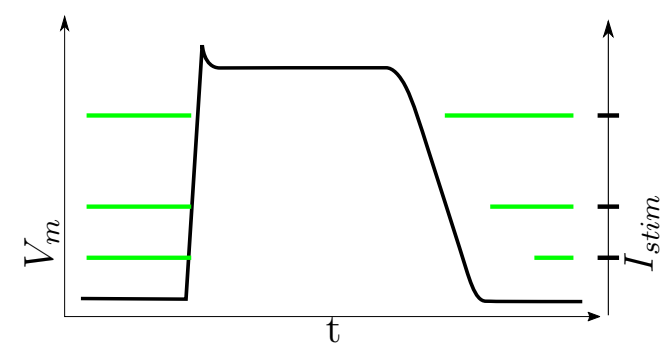

Figure 3.7.: Stimulus Dependence of Excitability - Schematic time course of the membrane potential of a single ventricular myocyte. Within the green intervals a new action potential can be triggered if a current as indicated to the right is applied. For stronger currents the excitable window reaches deep into the tail of the action potential. Virtual electrodes behave in a similar way.

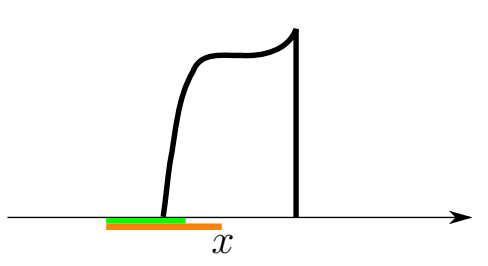

(a) $\mathrm{t}=0$

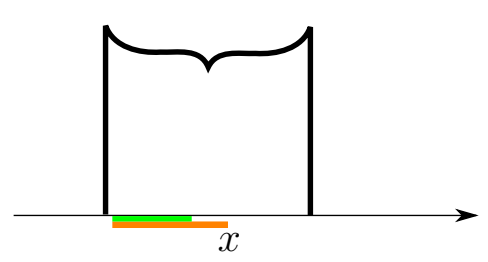

(b) $\mathrm{t}=1$

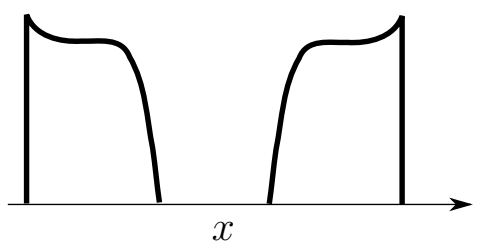

(c) $\mathrm{t}=3$

Figure 3.8.: Vulnerability - Schematic of the membrane potential as a function of space. Time is in arbitrary units. The wave is moving from left to right. The location of a cathode is indicated in orange. The excitable window with respect to the assumed stimulus is shown in green.

a): The wave is traversing the location of the electrode, one part of the electrode is in the excitable region the other is not.

$b)$ : At $\mathrm{t}=2$ a stimulus is applied via the electrode, the tissue in the excitable region undergoes another action potential and a second wave forms that travels in the opposite direction.

c): Both waves propagate independent of each other. 


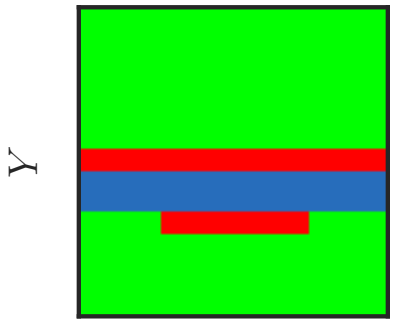

$X$

(a) $t=0$

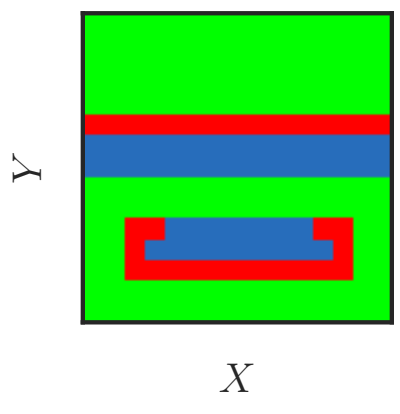

(c) $t=2$

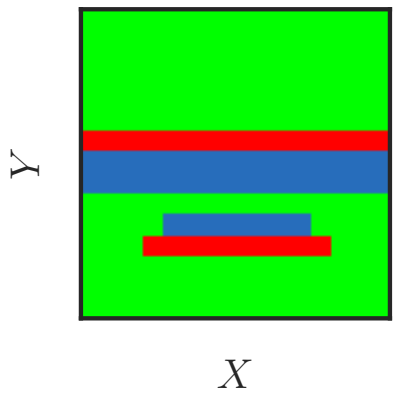

(b) $t=1$

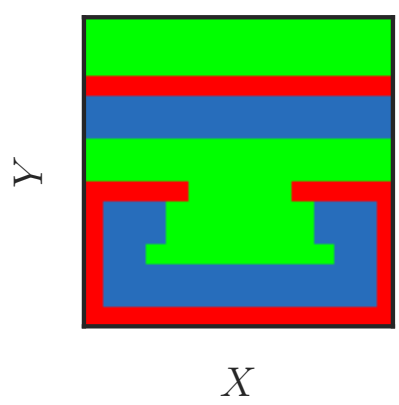

(d) $t=4$

Figure 3.9.: Vulnerability in 2d - Formation of a double spiral as a result of stimulation in the wave tail.

a): A stimulus is applied directly in the tail of the wave.

$b)$ : Due to the still refractory area behind it the forming wave travels downwards away from the stimulus site. At the edges of the front the excitation additionally starts to spread to the sides.

c): The sides of the wave start to curve inwards, flow around its own refractive tail and form two spiral heads.

d) Once the reentry around the tail is complete a stable double spiral is formed.

\subsection{Spiral Pinning}

It is know that anti tachycardia pacing cannot terminate all cardiac arrhythmias ([23]), a fact which in conflict with the observation made in section 3.2. One possible explanation for this failure of overdrive pacing can be found by considering the tendency of spiral waves to attache to conduction heterogeneities (see e.g. [88] or [67]): Any spiral wave that is anchored by inexcitable or less excitable obstacle must obtain a protection against simply being driven out of the domain $([125], 95])$. 


\section{The Heart as Excitable Medium}

This follows from a purely topological argument and the observation that spiral arms can only unattache by colliding with their mirror image ([95]). Waves coming from the pacing site to the pinned spiral must flow around the pinning obstacle (see figure 3.10), but if there is already an anchored spiral this means that there is an an odd number of spiral arms attached to the heterogeneity, and it follows that after the pacing is stopped one arm must stay pinned (see figure 3.11 for an illustration).

This line of thoughts can be formalized by noticing that spirals in general can be assigned a topological charge $S \in\{-1,1\}$, where the sign depends on the sense of rotation. Cast in this framework the unpinning by collision becomes the annihilation of two objects of opposite signature. Despite this annihilation the total charge in the domain stays constant and the system obeys a topological charge conservation law ([122]).

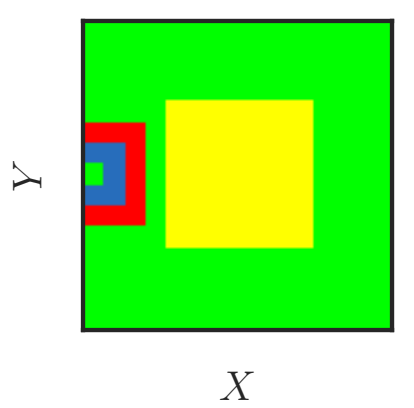

(a) $t=0$

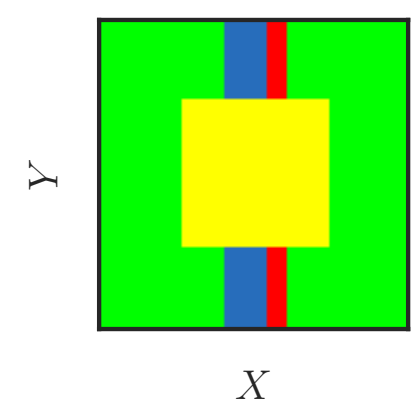

(b) $t=8$

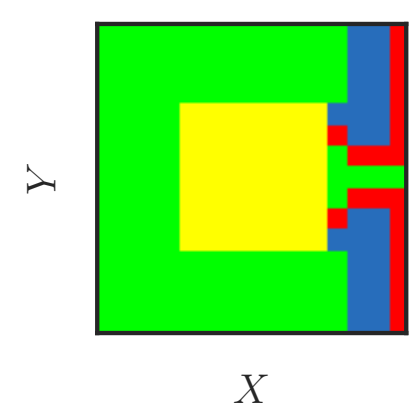

(c) $\mathrm{t}=15$

Figure 3.10.: Wave Flowing around a Heterogeneity - A wave coming from the left collides with the central heterogeneity (shown in yellow) and splits into two arms both attached to the obstacle $(b)$. The arms can only unattache by colliding with each other $(c)$. 


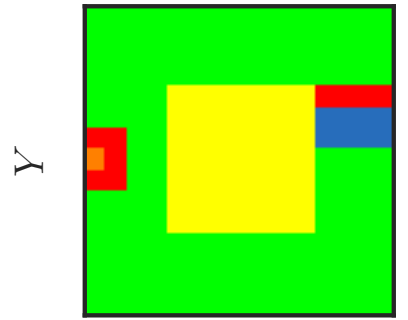

$X$

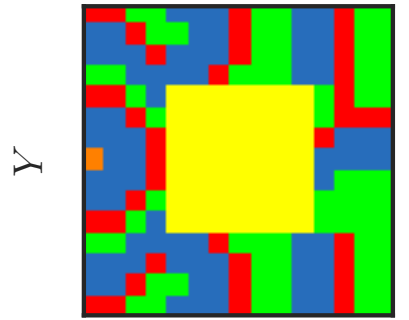

$X$

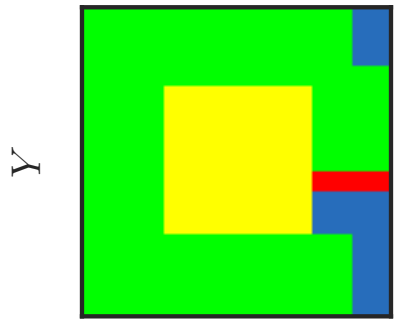

$X$
(a) $\mathrm{t}=0$
(b) $t=50$
(c) $\mathrm{t}=75$

Figure 3.11.: Topological Charge Conservation - A spiral wave attached to a heterogeneity in the presence of a periodically pacing electrode. Waves split at the obstacle, flow around it and unpin by collision with their mirror image on the other side. Since the original spiral lacks a counter part, one spiral arm is left pinned after the pacing is switched off. As spirals can be assigned a discrete topological signature, this phenomenon is called topological charge conservation.

\subsection{Spiral Unpinning}

As spiral pinning is assumed to stabilize cardiac arrhythmias much research has been devoted to the study of unpinning in silico (see [15] for a review). Here the mechanism proposed in [95] and experimentally reproduced in [114] and [30] shall be sketched briefly. It is based on the observations that using electrical fields the pinning center itself can be used as a virtual electrode. The procedure is visualized in the figures 3.13 and 3.12 .

If an electrical field is applied at the right instance of time, the virtual cathode forms in the vulnerable window of the action potential along the boundary of the pinning center. This creates two topological charges, one that stays attached to the obstacle and a second one that moves away from it. The newly created pinned spiral rotates around the obstacle in the opposite direction of the original one and once the two attached spiral arms collide both will be unpinned. 


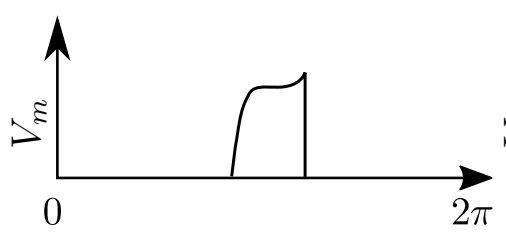

(a) $t=0$

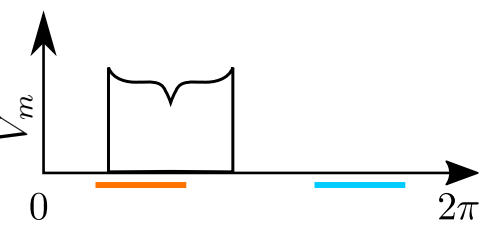

(b) $\mathrm{t}=1$

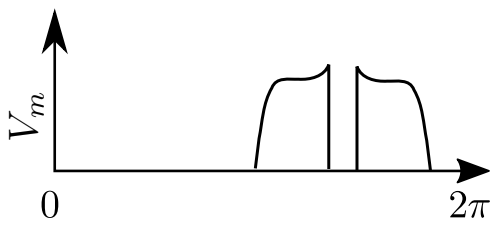

(c) $\mathrm{t}=2$

Figure 3.12.: Unpinning via the Vulnerable Window I - Schematic of the membrane potential as a function of space along the boundary of the pinning center. Periodic boundary conditions apply. The location of the virtual cathode is indicated in orange, the virtual anode is shown in cyan, as both form on opposing sides of the obstacle they are exactly $\pi$ apart.

a): The pinned spiral is traveling along the boundary from left to right.

$b)$ : At $t=1$ an electric field is applied. This leads to the formation of virtual cathode and anode on opposing sites of the boundary. If the virtual cathode forms in the vulnerable window a second spiral is created that propagates along the boundary to the left.

c): Due to the periodic boundary conditions both waves collide, their topological charges annihilate and the original spiral becomes unpinned. 


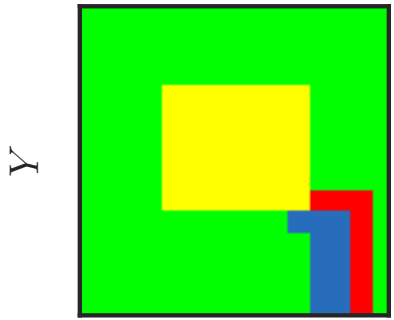

$X$

(a) $t=0$

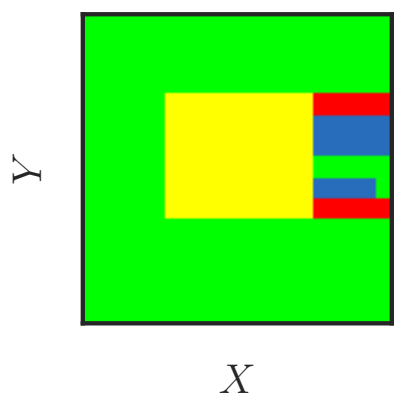

(c) $t=5$

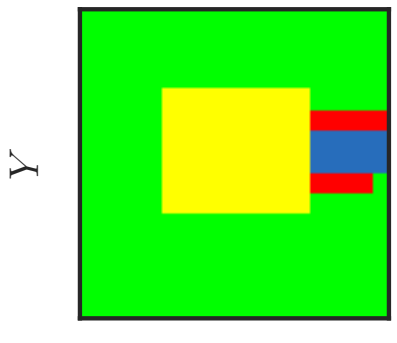

$X$

(b) $t=4$

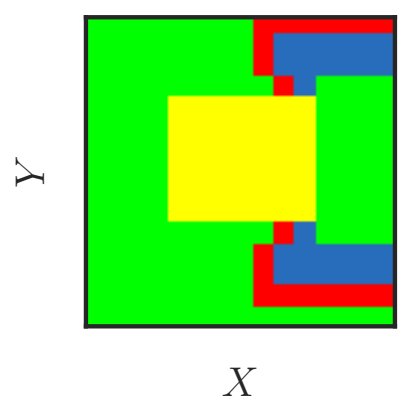

(d) $\mathrm{t}=9$

Figure 3.13.: Unpinning via the Vulnerable Window II - Unpinning of a spiral wave in two dimensions.

a): A spiral wave pinned to an inexcitable obstacle.

b): In the wake of the spiral wave a stimulus is applied and a wave nucleates.

c): The newly formed wave forms two spiral heads, one attached to the obstacle one free.

d): The free spiral head collides with the system boundary, two pinned spirals with opposite sense of rotation are left. Once these two collide the original spiral will be unpinned.

\subsection{Unpinning by Multiple Shocks}

The unpinning described in the previous section depends on the correct timing of the electric field pulse with respect to the location of the wave on the pinning center. This location cannot be known in the clinical setting, since excitation waves on the heart are as of now unobservable in vivo. It is therefore necessary to get rid of this sensitivity to timing before an antiarrhythmic therapy can be based on this mechanism.

To treat this problem [14] adapted ideas previously developed in [46] and introduced 
the phase transition curve (PTC) of a pinned spiral wave. The authors noticed that a wave pinned to an obstacle can be viewed as an oscillator by treating its location on the boundary as phase, given that the period of revolution is constant. Interaction of the spiral with waves emitted from the virtual electrodes along the heterogeneities circumference can then be analyzed based on the shift they cause in oscillator phase. The phase transition curve finally specifies the functional relation between the phase of the spiral at time of the stimulus and its phase one period later $\phi_{n+1}\left(\phi_{n}\right)$ (see figure 3.14 for a qualitative image of a PTC).

\subsubsection{The Phase Transition Curve of a Pinned Spiral}

The qualitative features of the curves that [14] observed in silico can be reproduced in a model that only considers the simplified properties of the action potential introduced in figure 3.1, it is motivated to some extend by a similar construction shown in [53]. This axiomatic model is based on four considerations. Effects of wave speedup due to hyperpolarization are not considered, the spiral propagates with constant velocity around the boundary. The location where the virtual cathode forms is fixed and depending on the position of the action potential with respect to the virtual cathode only three things can happen:

I The cathode forms in uniformly inexcitable tissue and nothing happens.

II It forms in the vulnerable window, a single new action potential forms and travels around the circumference in the opposite direction away from the original one.

III The cathode forms in uniformly excitable tissue. Two additional action potentials nucleate and propagate in opposite directions around the obstacle.

I, II, and III correspond to three different regions in the PTC, which are labeled accordingly. In region $\mathbf{I}$ the spiral keeps rotation with constant velocity and is at the same location one period later. The phase transition curve in this region is a subset of the identity.

In region II the second formed action potential travels around the object and collides with the original pinned spiral which leads to unpinning. Since one period later there is no wave attached no phase can be defined. The PTC is interrupted in this region.

Finally in region III the two formed waves move around the pinning center, one of them collides with the original one and unpins while the other one stays attached. The remaining spiral originated at the cathode at time of the stimulus and one period later it appears that the wave jumped to this location, regardless of its position at time of the stimulus. This leads to a plateau in the PTC at the phase that corresponds to the 
location of the cathode.

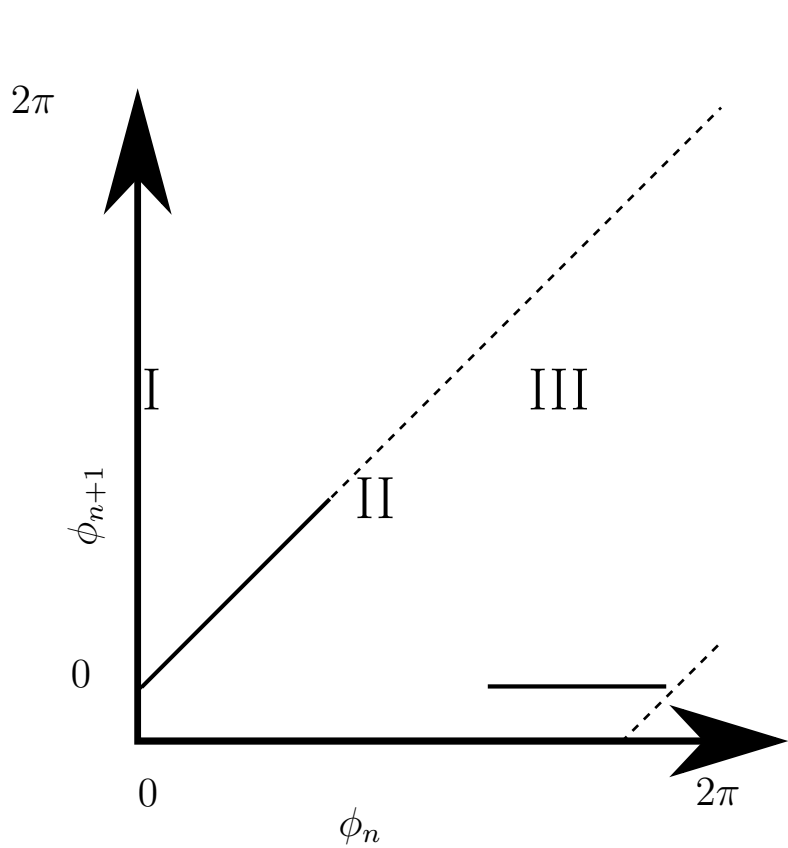

(a)
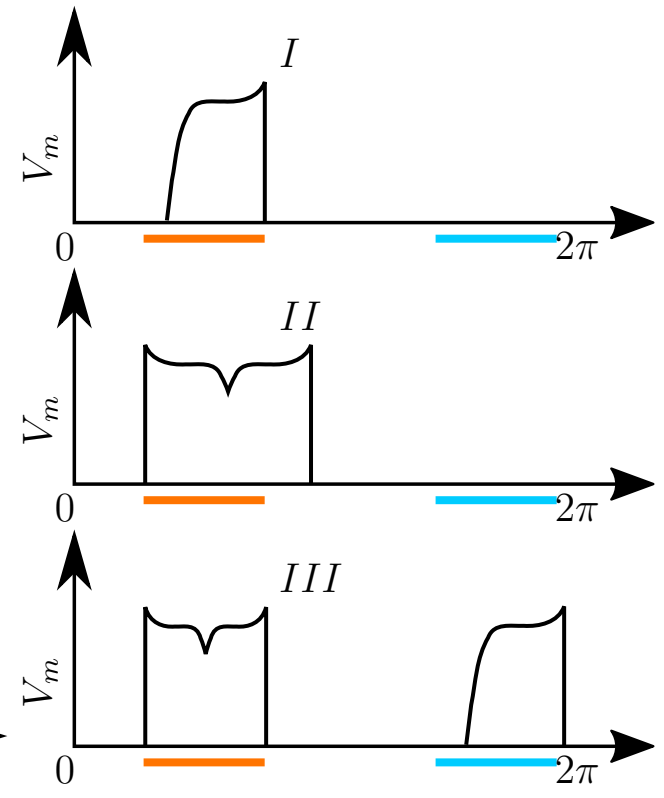

(b)

Figure 3.14.: Qualitative Phase Transition Curve - The three differnt regions of the PTC and the corresponding tissue response at the cathode.

a): The phase one period after the stimulus as a function of stimulus phase. Zero has been set to the location of the cathode. The phase transition curve is shown in black, the identity is indicated as perforated line. The labeled regions correspond to the situations shown to the right.

$b)$ : Schematic of the membrane potential as a function of space along the boundary of the pinning center. Periodic boundary conditions apply. The location of the virtual cathode is indicated in orange, the virtual anode is shown in cyan.

In I the action potential is on top of the cathode, no new wave can form. The PTC lies on the identity.

In II it is located in the vulnerable window, and unpinning occurs. $\phi_{n+1}$ is undefined for this case, and the PTC is interrupted.

In III two counter rotating waves form, one collides with the original action potential and unpins. This leads to an apparent jump of the wave to the location of the cathode one period later, this causes a plateau in the PTC.

As the regions arise directly from the properties the action potential their ordering (I,II,III) is fixed to by the ordering of the corresponding parts (excitable window, vulnerable window, inexcitable window). It can however be cyclically permuted by choosing 
a different origin for the $\mathrm{PTC}^{2}$. Since the construction of the phase transition curve in this way is an inherent geometric procedure, the reader is referred to figure 3.14 at this point.

Depending on the obstacle size and the used field strength the shape of the curve will vary. For weaker fields the vulnerable window will decrease and finally vanish (compare figure 3.7 and [53]) making region II disappear. Similarly for smaller obstacles the action potential will cover larger parts of the boundary, making region III shrink.

Phase transition curves observed experimentally in cell cultures and simulation are in good agreement with the curve shown in figure 3.14 (see figure 3.15 and [30] for experimental data and [14] or [16] for simulations), although region III can be seen to bend downwards near region II.

An explanation for this bending is conduction velocity restitution, the property of cardiac tissue to conduct excitation in a rate dependent manner. The velocity of waves decreases thereby with decreasing interval between repeated excitation. Within region III the distance at time of shock between virtual cathode and pinned wave increases from left to right. Waves originating at the virtual cathode encounter therefor tissue that had less time to recover when pulses are given at phases on the left edge of region III (see figure 3.16 for an illustration), the resulting wave slowing causes the PTC to bend downwards.

${ }^{2}$ Choosing a different origin corresponds to shifting the curve to the left or the right, but parts that are shifted across one boundary must reenter from the other since the axis is periodic. 


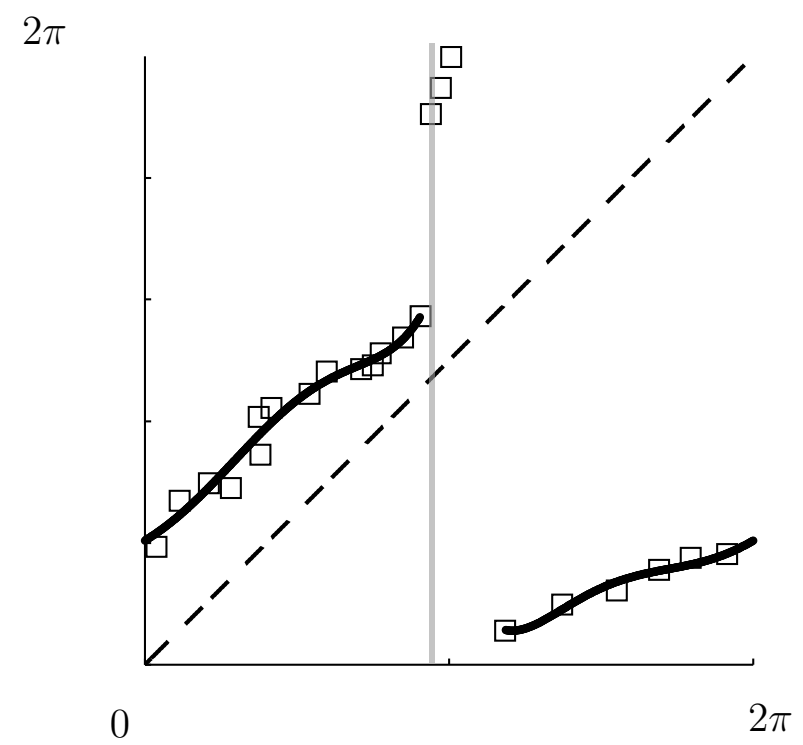

Figure 3.15.: Experimental PTC - Phase transition curve of a pinned spiral in a cell culture of neonatal chicken cardiomyocytes. Figure modified from [114] with permission of the authors. Measurements are shown as squares, the small vulnerable window is shown as gray bar. The black lines interpolate the data points.

The three points near the top of the figure, directly right to the vulnerable window are due to wave slowing as discussed in the text.

For experimental consideration the authors defined the phase as angle between the wave front and a point on the circumference, rather than by parametrization. In consequence of the not perfectly circular boundary and a highly heterogeneous cell culture, the velocity defined in this units was not the same on all parts of the heterogeneity. A region with higher wave speed causes the black line left to the vulnerable window to be shifted upwards. Apart from this shift the curve fits well with figure 3.14: A region close to the identity, an unpinning region and a plateau are visible.

\subsubsection{An Iterated Map for Multiple Shocks}

Once the phase transition curve of a pinned vortex is known, it can be used to investigate shock strategies.

To this end notice that the position of the phase transition curve can be vertically shifted by using $\phi_{n+1+\tau}\left(\phi_{n}\right)$, the phase of the spiral $1+\tau, \tau \in[-1, \cdots, 1)$ periods after the shock, rather than $\phi_{n+1}$ as dependent variable. This follows from the fact that the spiral will rotate with constant velocity after the shock and cover $2 \pi \tau$ additional phase units in the 


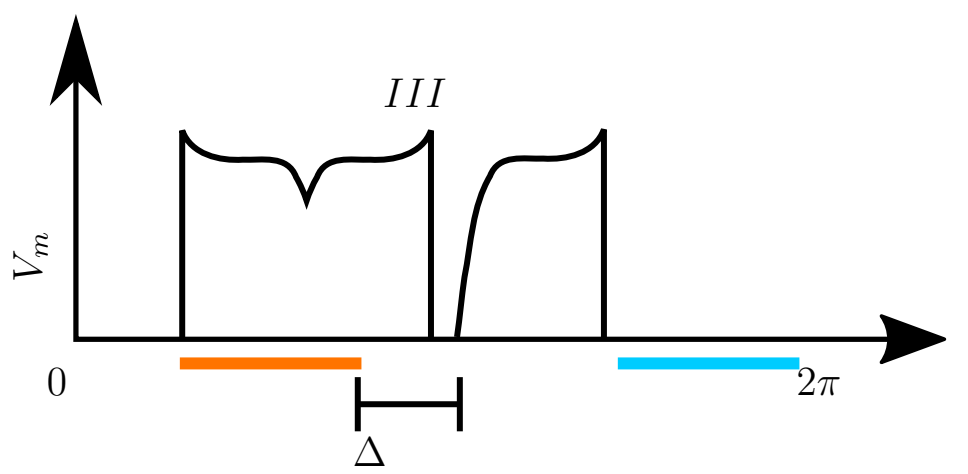

(a)

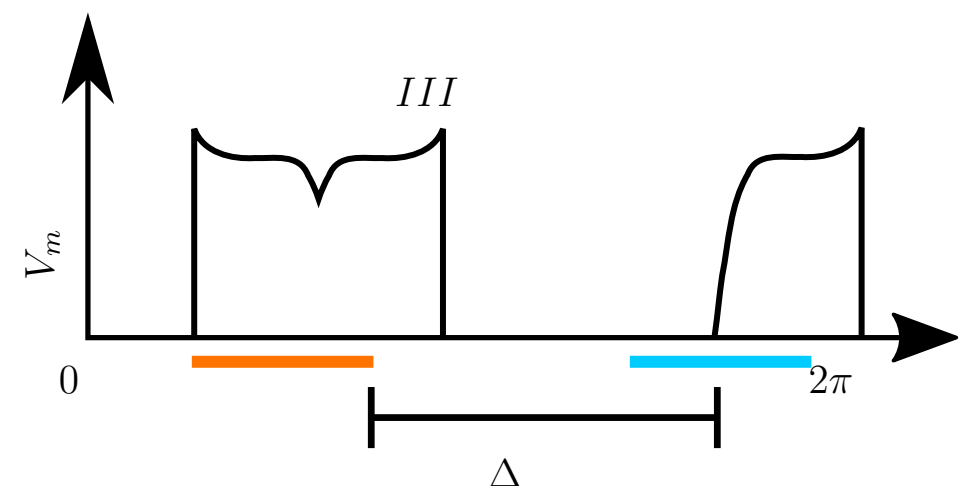

(b)

Figure 3.16.: Wave Slowing - The different distance between the cathode and the pinned wave causes the edge of region III to bend down.

As a response to a field, two new waves form at the virtual cathode. One travels around the boundary and collides with the original wave, while the other follows the original in its wake.

a): On the left edge of region III the distance $\Delta$ between the original wave and the one shadowing it is small. Consequently, conduction velocity restitution will slow down the new wave, causing the edge of the PTC to bend downwards.

$b)$ : Deeper within the region this effect is less pronounced as $\Delta$ increases and the tissue has more time to recover. 
additional time. Since this is the case for all phase values it leads to a displacement of the whole curve. Positive $\tau$ will move the curve upwards, while negative $\tau$ will cause a downward shift.

In the special case of $N$ periodic shocks with period $T(\tau+1)$, where $T$ is the period of the spiral, the phase after each shock can be computed by iterating $\phi_{n+1+\tau}\left(\phi_{n}\right)$. This can conveniently be visualized as a cobweb diagram (see figure 3.17 and [114]).

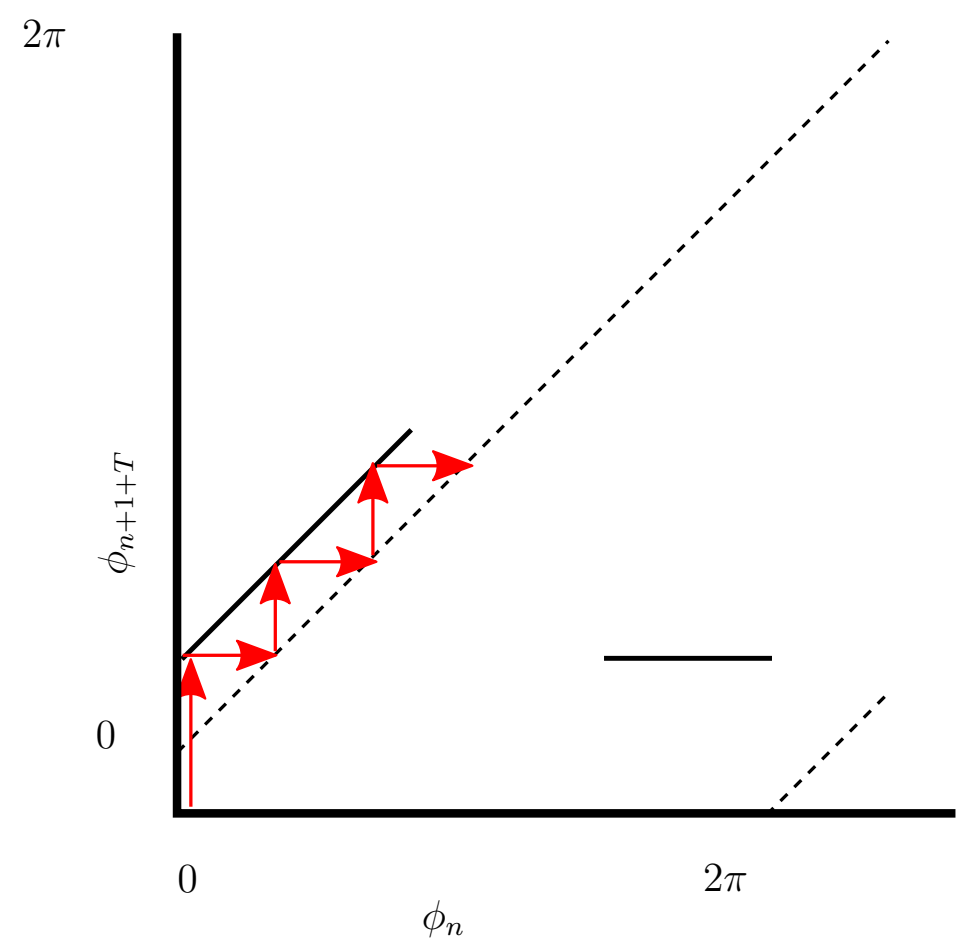

Figure 3.17.: Cobweb Diagram of Periodic Pulses $\tau>0$ - Using the cobweb construction the phase after each pulse can be conveniently visualized. PTC in black, the identity is indicated as perforated line. Periodic boundary conditions apply to all axes. The curve is shifted upwards due to a positive $\tau$. Starting at $\phi_{0}=0, \phi_{1}$ can be directly read off the graph. $\phi_{2}$ can then be constructed by moving to the right until the point $\left(\phi_{1}, \phi_{1}\right)$ on the identity is encountered. Moving upwards from there to the PTC one can read off the point $\phi_{2},=$ $\phi_{n+1+\tau}\left(\phi_{1}\right)$. Iterating this procedure generates the phases at the successive shocks. Connecting the intermediate steps of the integration, creates the web like pattern that gives this type of diagram its name (shown in red). In the shown example the fourth shock falls in the vulnerable window, the wave would therefore be unpinned.

Looking at figure 3.17 it seems that, given a sufficient number of pulses is applied, the spiral will always be unpinned, because at some point the system will end up in the displayed staircase like motion along the PTC. This concept is called phase scanning and 


\section{The Heart as Excitable Medium}

does indeed provide a method to deterministicly unpin a rotor, albeit only in the absence of stable fix points in the iterated map. Such points arise when the PTC intersects the identity, and the modulus of the derivative at the point of intersection is smaller than 1 ([127]). Looking at figure 3.14 this situation could easily arise if $\tau$ was chosen to be negative. In this case the plateau would intersect the identity and the arising fixpoint would be stable, since the derivative within the plateau vanishes (see figure 3.18).

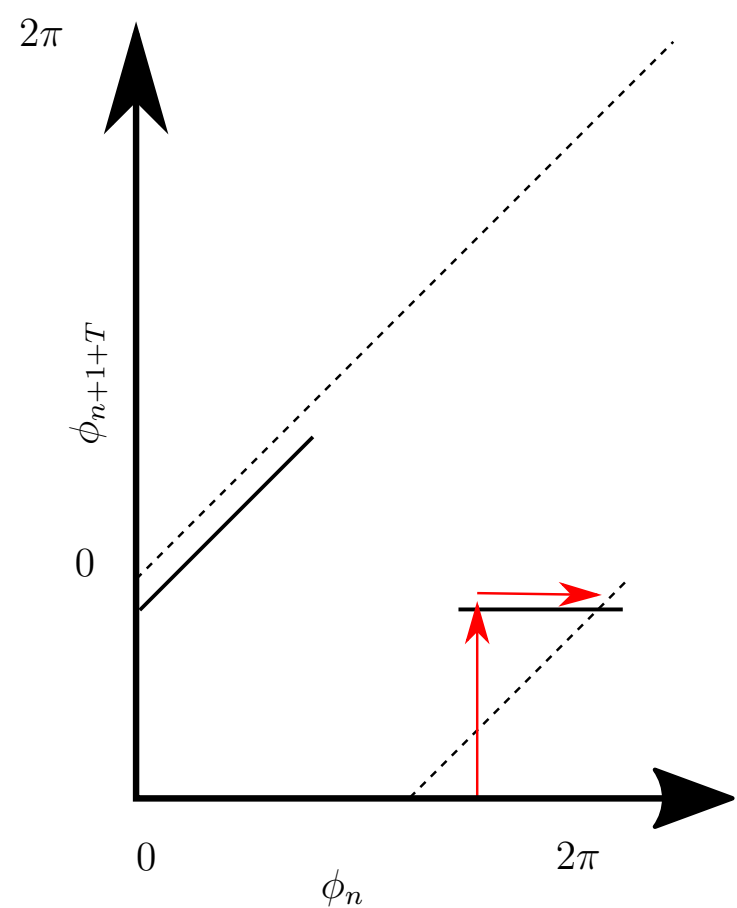

(a)

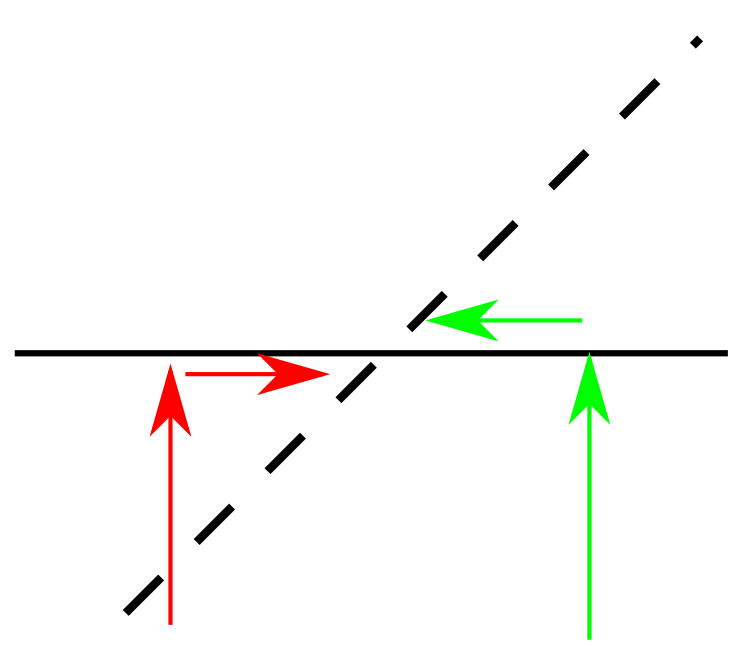

(b)

Figure 3.18.: Cobweb Diagram of Periodic Pulses $\tau<0$ - Definitions as in figure 3.17, but this time the curve is shifted downwards due to a negative $\tau$. As the axes are periodic, this causes an intersection of the plateau with the identity, and hence a fixpoint in iterated map $(a)$. Phases left and right of this intersection are mapped onto it by the cobweb construction, the fixpoint is therefore stable $(b)$

[14] therefore predicted that phase scanning could be reliably achieved by giving shocks with a period slower than the spiral frequency, meaning by choosing a positive $\tau$. This result is slightly counter-intuitive, since one would naively expect that faster pulse would act similar to ATP, but it is in agreement with observations made in multi shock defibrillation in living animals and in vitro hearts. 


\section{Arrhythmias}

The pumping power of the heart depends on the correct timing of the contraction of its different parts, hence disturbances of the activation spread or the pacing will decrease cardiac performance. Many of these irregularities can be noticed by examination of the electrocardiogram or ECG, which measures an integral quantity related to the far field of the electrical activity.

Two interesting dynamical states which can be recognized in the ECG are labeled tachycardia and fibrillation.

Characterizing feature of tachycardia is a rapid but periodic ECG signal; whereas the signal produced by fibrillation is irregular. A transition from the former to the latter can often be observed and in vitro is apparent to the naked eye, since the tissue movement changes from quivering to almost motionless as it occurs.

\subsection{VF: Ventricular Fibrillation}

Fibrillation is a self sustaining state of disorganized electrical activity in the heart muscle, that can occur in the ventricles as well as the atria [34. As a consequence of this disorganization the local dipoles that give rise to the ECG do not align globally and the electrocardiogram presents as a fluctuation highly irregular signal with broad frequency spectrum and low amplitude (see. [45] and figure 4.1).

For the patient fibrillation is associated with a loss of cardiac output which in case of ventricular fibrillation (VF) is life threatening, since a complete breakdown of the circulation is the consequence. In fact VF is the main mechanism behind sudden cardiac death $([108])$, which in turn is the leading cause of mortality in Europe ([2], [1]).

Currently the only reliable therapy to treat fibrillation is the application of an electrical field $\left(7 \frac{\mathrm{V}}{\mathrm{cm}}\right)$ across the heart ([36]), either via an external defibrillator or via an implanted cardiac defibrillator (ICD). These defibrillation shocks impose a high therapeutic burden on the patient as they are painful ([119]) and damaging to the tissue ([134]). Reducing the field strength necessary for defibrillation is therefore one of the great challenges of 


\section{Arrhythmias}

cardiac research $([74],[36])$.

From a pattern formation point of view fibrillation consists of multiple reentrant waves on the heart muscle that result from repeated spiral wave break (see 3.2 and [47]) and ideas originating from the field of excitable media research have successfully been applied to the problem of fibrillation prevention [26]) and termination ([74]).

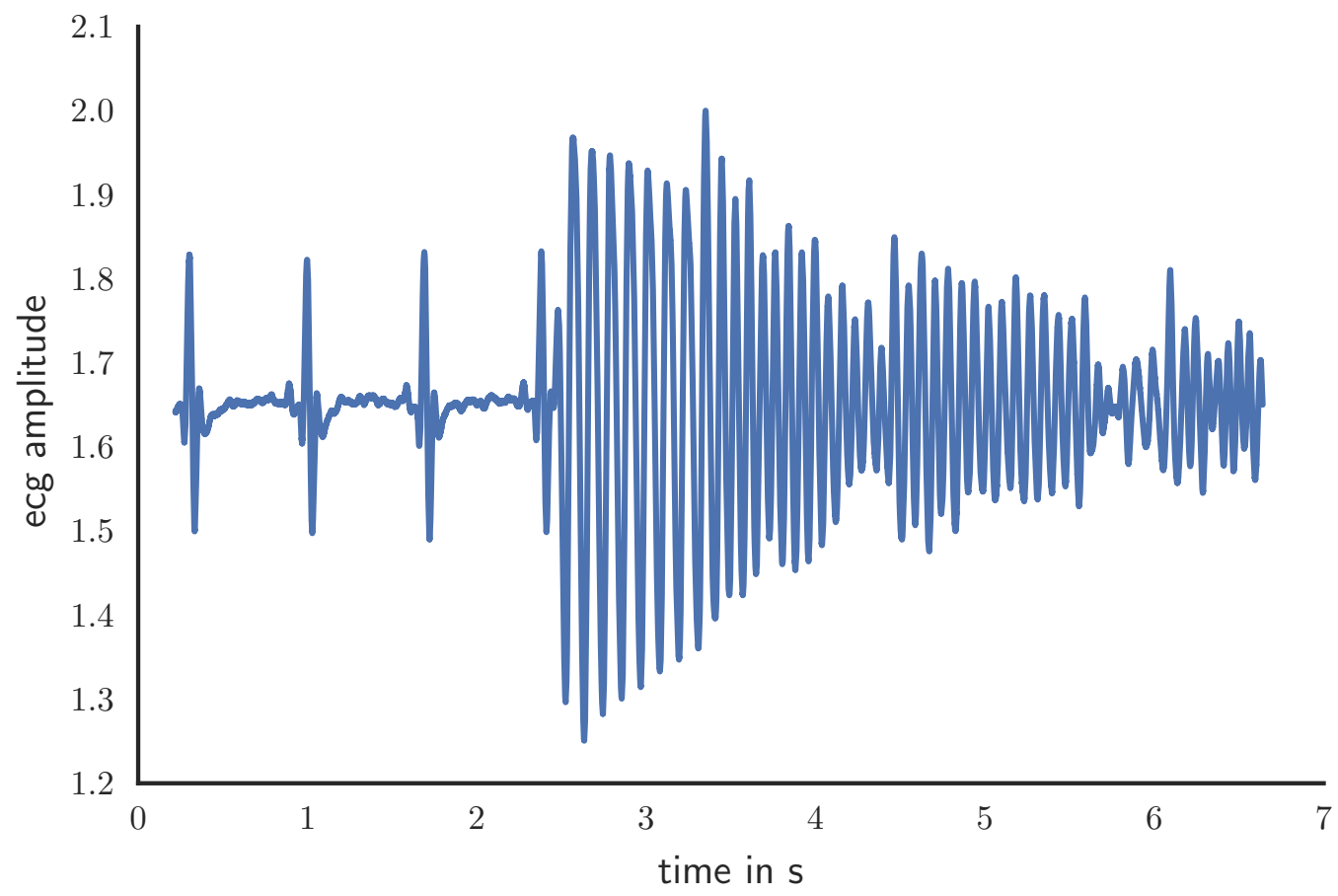

Figure 4.1.: Transition between VT and VF - ECG obtained using our experimental setup. Data courtesy of Dr. Alexander Schlemmer. Normal sinus rhythm spontaneously converts to VT after $2.5 \mathrm{~s}$. This VT then further deteriorates than to VF after 1s. Note the lower amplitude and higher amount of fluctuations in VF. A similar mechanism is thought to underlie most cases of sudden cardiac death $([108])$.

\subsection{VT: Ventricular Tachycardia}

Compared to VF, ventricular tachycardia is a more ordered: Where fibrillation presents as a state with multiple short lived wavelets on the heart muscle, VT is associated with a single periodic pattern. This high degree of spatio-temporal organization during VT leads to well defined periodic ECGs and a coarse distinction between VT types can be easily made from the ECG. Episodes with constant amplitude are called monomorphic VT, while VT is called polymorphic if the ECG pattern appears regular but amplitude 
modulated by a slower frequency. Monomorphic VT is thought to arise from stationary reentries, while polymorphic VT is believed to arise when spirals move freely across the heart $([31])$.

In addition to this rough sorting, a much more fine grained analysis of the ECG is possible based on characteristic patterns in these electrocardiograms. These are called morphologies, and highly trained medical doctors can in a few special cases narrow down the location of a VT's driving focus by analyzing a 12 lead ECG and comparing its morphologies to known patterns ([61, [33]).

VT can occur as a complication in all myocardial diseases but healed myocardial infarction is the most common clinical situation that gives rise to sustained ventricular tachycardia ([34]). Despite the clear observed correlation the mechanism by which infarction scars promote VT is still unknown ([7]), two major hypotheses being delayed conduction through the scar ( [120]) and pinning of a spiral wave by scarred tissue $([104])$.

Although not immediately life threatening itself a transition from spontaneous VT to deadly VF is thought to be the most common course of events that leads to fibrillation ([108]), a transition like this is shown in figure 4.1. Suppressing VT would accordingly be a much desired supplemental therapy for high risk patients with an implanted defibrillator, since this could reduce the number of necessary defibrillation events. Unfortunately pharmacological therapies so far failed to achieve this goal (e.g. [94] where the patient mortality was actually increased due to side effects), and surgical intervention is not effective in all patients ([130]). A supplemental bio-electrical therapy that specifically targets VT and is less damaging and less painful than defibrillation would therefore be a valuable addition to the existing treatment methods.

\subsection{Cardioversion}

Cardioversion is the general medical term for an intervention that restores normal heart rhythm after an arrhythmia. A broad spectrum of methods exist that reaches from injections of antiarrhythmic drugs to shocks delivered from external defibrillators. For currently used bio-electric therapies ${ }^{1}$ one distinguishes between triggered and untriggered cardioversion.

Triggered cardioversion refers to the delivery of a low field strength shock at a specific phase of the observed ECG, usually the peak or close to the peak of the ECG, a method developed to avoid the induction of fibrillation by applying shocks in the vulnerable window. It is currently only used to treat atrial arrhythmias or well tolerated abnor-

\footnotetext{
${ }^{1}$ the treatment of arrhythmias with electrical fields and currents
} 


\section{Arrhythmias}

mal ventricular rhythms such as heamodynamically stable tachycardia, fields employed for triggered cardioversion are in the region of $1 \frac{\mathrm{V}}{\mathrm{cm}}$. Contrary to that untriggered cardioversion refers to any shock that is given without regard to the ECG and therefore is synonymous with the defibrillation. Currently used defibrillation fields are about $7 \frac{\mathrm{V}}{\mathrm{cm}}$ (see [56] for an overview).

\subsubsection{Determinism in Cardioversion}

Cardioversion of fibrillation as well as tachycardia is probabilistic, where the observed termination probability depends on the field strength in a non linear but monotonic way ([75]). The origin of this seeming non determinism is not fully understood but is attributed to the complex interplay between reentrant waves and the field strength dependent excitation source density ([55]). Taking a non linear dynamics point of view, the spatially extended system that is the heart travels along a trajectory in phase space entering and leaving regions that are differently susceptible to cardioversion.

As of now no method is known to distinguish the susceptible from the less susceptible states. However, contrary to fibrillation tachycardia is a periodic excitation pattern, and hence periodically traverses the different regions of its phase space orbit. Different regions on that orbit therefore map to different regions in (oscillator) phase. Indeed, phase dependence has been predicted based on simplified numerical systems ([16], [14]) and was reported for animal models ([103], [104]).

Another source of an apparent lack of determinism in VT cardioversion is the possible existence of different tachycardic states in the same heart. [104] manually distinguished on average 11 different VT morphologies per heart in a series of rabbit experiments. Possible phase dependencies can therefore be masked if one fails to distinguish between these coexisting states.

\subsubsection{Mechanisms of Defibrillation}

\section{Conventional Defibrillation}

For defibrillation to be guaranteed successful it must terminate all existing fibrillatory excitation while not creating a new reentry. Situations that deviate from this two requirements might still lead to successful termination of the arrhythmia but in a manner dependent on the pre shock distribution of excitation and refractoriness, which accounts for the observed fluctuations in defibrillation success. 
Both requirements can be understood using the virtual electrode framework. For this purpose recall that in section 3.1.2 it was pointed out that cardiac tissue polarizes near heterogeneities in response to an applied electrical field. Depolarized areas are either excited or existing excitation is prolonged, hyperpolarized areas are deexcited and wave speed increases for a brief period after the hyperpolarization.

Monophasic Shocks Simple electric fields applied across the heart are called a monophasic shocks. A successful monophasic shock achieves the two criteria defined before the following way: Firstly, the state of each cell, either excitable or excited, is completely determined by the forming de- and hyperpolarization zones. This can be achieved by applying strong fields, as the extend of the virtual electrodes depends on the field strength. Secondly, the speedup of waves within hyperpolarized regions is sufficient to guarantee that waves forming in depolarized tissue traverse these regions in less than the refractory period. This prevents the creation of new reentrant waves, by causing conduction block along all possible reentrant circuits (see [102]).

Biphasic Shocks The minimum field strength for the success of the method described in the last paragraph is determined by the needed speedup in the hyperpolarization zones. To circumvent this and operate at lower fields, current defibrillators apply an additional weaker shock with reversed field direction, which causes an inverted pattern of virtual electrodes. Since the second shock has a lower field strength the activation that was caused by the first one is not suppressed, while the hyperpolarization zones are nevertheless excited. This again closes possible pathways of reentry and thereby prevents the reinduction of an arrhythmia ([102])).

\section{Unpinning}

The mechanism described in the previous section can describe defibrillation at large fields, where the virtual electrodes dominate the dynamics. To understand defibrillation at low field strengths or cardioversion of VT, a better model for the interaction between virtual electrodes and existing waves is needed. Such a mechanism has been proposed based on general models of excitable media.

Contrary to what is observed in fibrillation, in these models spiral wave driven chaos on finite domains can be terminated by local overdrive stimulation. To consolidate this observation with cardiac dynamics it has been proposed that heterogeneities in the heart anchor spiral waves thus increasing their stability (see e.g. [15]) a feature which can be reproduced in reaction diffusion systems ([67]) as well as in in vitro ([29]) and in silico models of cardiac tissue ([14]). Based on this it has been suggested that removing the anchored or pinned spirals from their pinning center supports defibrillation ([95]) by 


\section{Arrhythmias}

reducing the needed field strengths, with some authors suggesting that defibrillation equals unpinning (see [103] ). Several cardioversion methods have been developed based on the unpinning framework. All of them depend on the phase transition curves of the assumed pinned spirals and all could be interpreted in the iterated map framework of section 3.6.2.

LEAP: Low Energy Antifibrillation Pacing 74 reported $80 \%$ reduction of needed defibrillation energy when using five periodic pulses with a shorter period than the arrhythmia, the suggested mechanism being unpinning by excitation in the vulnerable window of the pinned spirals. [54] later reported that periodic pulses given at a frequency slower than the arrhythmia frequency were superior to faster pulses, although the energy reduction observed in [74] could not be reproduced there. The author speculated that a fixpoint in the phase transition curves of pinned spiral fragments, similar to the one discussed in 3.6.2, could be the reason for this discrepancy.

Multistage Electrotherapy [57] observed 98\% reduction in necessary energy when terminating tachycardia by a multistage shock protocol. The protocol prescribed first the administration of three shocks within one VT period and than a series of lower amplitude shocks followed by ATP. According to the author it was motivated by the assumption that spirals should be first unpinned and then driven into the system boundaries. The application of the three faster shocks in the unpinning phase makes the implicit assumption of the absence of a fixpoint.

Synchrolation Finally, work by Henrik tom Wörden, Dr. D. Hornung and myself suggests that first applying multiple weak shocks at a frequency higher then that of the arrhythmia and than a single stronger shock could lead to a reduction in the necessary field strength (unpublished work, patent pending [49]). The believed mechanism here is that all pinned spirals are first driven to the fix point in their PTC by the fast shocks (see section 3.6.2) and then simultaneously unpinned. 


\section{Models of Myocardial Infarction}

\subsection{Myocardial Infarction}

The heart has a high metabolic demand due to its constant contractions, this demand is met by a special distribution system attached to it, the coronary vasculature. Acute myocardial infarction occurs when one of the coronary arteries is occluded and tissue which is normally perfused by that artery becomes first ischemic and finally starts to die (see figure 5.1). Over the time course of several months the dead cells are replaced by connective tissue as the infarction enters the chronic phase. The result of this process is a mature myocardial infarction scar that penetrates the muscle and is highly arrhythmogenic $([128])$.

Myocardial infarction is present in $80 \%$ of all sudden cardiac deaths ([84]), and chronic myocardial infarction is the only known not congenital cause of repeated sustained ventricular tachycardia ([80]). At an incidence rate of 200-300 per 100.000 population per year and high probability of survival (80\%-90\% with a tendency to increase) the prevalence of chronic ventricular infarction in the general population is increasing, and so are consequentially the secondary diseases that follow from it ([106]).

Infarcts have some unique properties that are difficult to reproduce in animal models, some of which follow from the fast and effective modern medical care. Since most surviving patients receive emergency treatments, rarely all tissue that is supplied by the afflicted blood vessel dies. Therefore a boundary zone forms at the border between scar tissue and healthy myocard, containing damaged but viable tissue. This zone is thought to be one of the main breeding grounds for arrhythmias ([27]). For the same reasons within the scar surviving strains of myocard exist ([100]) which are of importance for tachycardia, since they are one possible reentry path.

Leaving this details aside, at its core the infarct scar is an area that is lacking in myocardial cells, and it has been shown that the infarct tissue is only weakly electrically coupled to the myocardium that surrounds it ([90], [113]). From a bidomain point of view, an infarct is hence furthermost a hole in the intra cellular space and as such may anchor spiral waves or act as virtual electrode. 


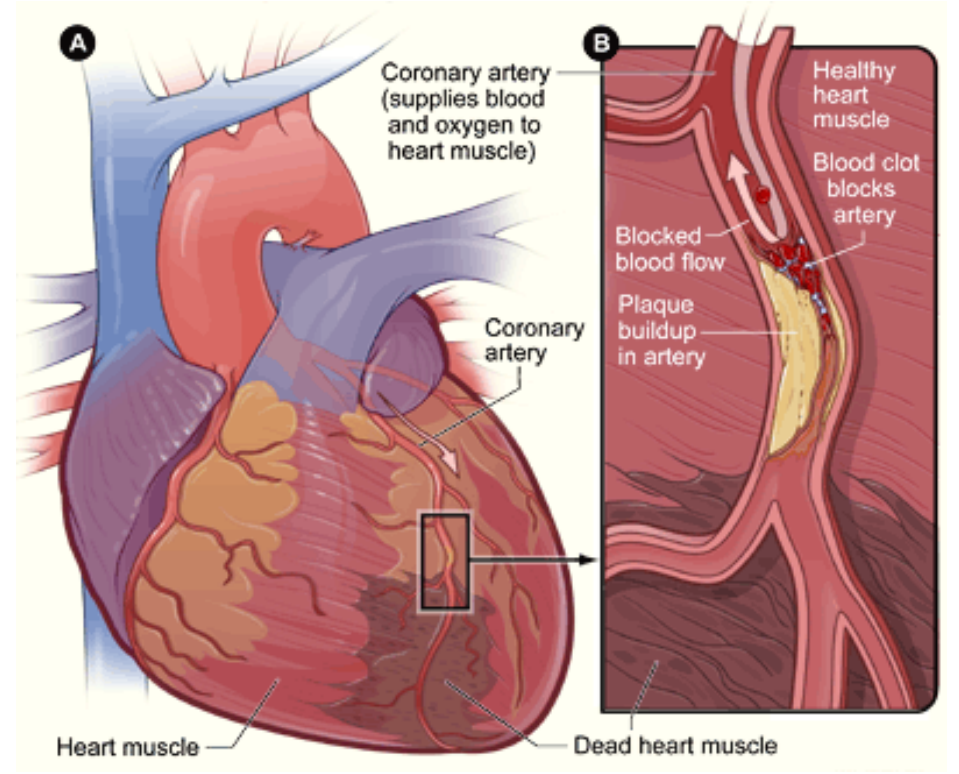

Figure 5.1.: Myocardial Infarction - A blockage directly below the second branch of the left anterior descending artery leads to acute ischemia in the region that is perfused by this blood vessel. The depicted infarct locating is identical to the one we investigated in pigs. Source: National Heart, Lung, and Blood Institute, National Institutes of Health.

\subsection{Models of Chronic Myocardial Infarction}

No animal model that fully reproduces chronic infarction in humans does currently exist, but a number of models that approximate some of its aspects have been described ([99]). All of these models have in common that a large heterogeneity is introduced into the cardiac muscle, that serves as a possible pinning center similar to the idealized heterogeneity presented in section 3.1 .

\subsubsection{Cryoablation}

Cryoablation is the process of locally killing tissue by cooling it below the melting point off water. This causes ice crystals to form within the cells and the vasculature, rupturing the cell membranes and inducing local ischemia (44]). Clinically it is used to remove the affore mentioned remaining viable cells within mature infarction scar, a process called infarct homogenization ([130]).

Two main parameters can be adjusted in cryoablation procedures: the cooling rate and the cooling duration. [44] found that increased rates kill cells more effectively and [60] 
suggested that the needed fast cooling could be achieved using liquid nitrogen, [52] finally observed that increasing the duration of the cooling and performing several freeze thaw cycles leads to higher amounts of necrotic tissue. The optimal method therefore seems to be repeated prolonged cooling by liquid nitrogen.

On cell level, both cell rupture as well as ischemia lead to gap junction uncoupling ([124] and [35]), which greatly increases the intra cellular resistance. From a bidomain point of view a cryoablation site is therefore again a hole and can be seen as a model for a myocardial infarction scar. Indeed [60] found that cryoablation sites acted as pinning centers in a porcine in vitro model.

\subsubsection{Ischemia}

Ischemia is a state where tissue is insufficiently supplied by oxygen and nutrients and metabolic wastes are only inadequately removed. It is often caused by vessel obstruction (e.g. acute mi) and causes gap junction uncoupling and finally cell death ([64]).

Interpreting ischemia in the bidomain model is harder than cryoablation or mature infarction, since it is by its nature not stationary! 1 . It has been observed that in the early phases of ischemia the extra cellular conductivity declines, since in this first phase the cells swell and reduce the extra cellular volume. This phase is then followed by a sharp increase of intra cellular resistivity caused by the onset of gap junction uncoupling ([121]). Although extra cellular resistivity remains elevated this massive decrease in intra cellular conductivity leads to a final situation that resembles the mature infarction case.

A special case of ischemia is reperfused ischemia, where the occlusion that induces the undersupply of the tissue is only temporary. In this case the tissue incurs a paradoxical damage due to the reestablishment of perfusion, the so called reperfusion damage. The mechanisms of this are as of now not understood, distribution of metabolic waste through the heart as well as the flushing of small amounts of coagulated blood into the capillaries are thought to be of importance ([135]). Especially the latter is of interest for this work as it is one major point of distinction between reperfusion with blood and saline solution. Both mechanisms again would lead to an increase of intra cellular resistivity. Several different reports have been made regarding the success and the appropriate duration of the occlusion in reperfusion models. [25] report that 14 out of 25 preparations of rabbit papillary muscle recovered perfectly after ischemia and reperfusion. But in this case the ischemic period was brief to the point where gap junction uncoupling was just setting in. Furthermore they induced ischemia in saline solution and not in blood. Contrary to that [77] report that in dog in vivo models of ischemia large portions of tissue that is viable

\footnotetext{
${ }^{1}$ The stationary phase of ischemia would be an infarction scar.
} 
at the end of the ischemic period looses viability in the first hours after reperfusion, and [135] state that this process is much faster in pigs where complete transmural necrosis is achieved after 90 minutes of ischemia.

Finally the hypothesis that ischemic zones act as pinning centers is supported by observation made in [111], where among several tried configuration the only one that reliably induced VT in a porcine model was a small ischemic zone and low doses of ajmaline. Furthermore [17] observed spiral pinning in an ionic model of ischemic tissue. 


\section{Methods}

An integral part of this thesis is the comparison of the time series obtained during the performed experiments using methods from chaos theory. Although theses tools are part of the domain specific knowledge of the field of non linear dynamics, they are less known in the cardiac sciences. This section will therefore provide background information for the quantities used in chapter 9 .

First a method to convert a scalar time series measured from a deterministic dynamical system into a probability distribution is introduced (6.1). Then a distance measure between thus obtained probability distributions is motivated (6.2), it will be used to introduce a notion of distance between VTs in section 9.3 .

In section 9.3.5 this procedure will later be compared to two other methods, the maximum correlation and a PCA based measure. The latter will be explained in 6.3.

The statistical tools needed for the mentioned comparison are introduced in 6.5 and 6.4). Finally an overview of the clustering method that will be used to group ventricular tachycardia into morphologies is given in section 6.6 .

\subsection{Delay Reconstruction}

Cardiac dynamics is deterministic, and the future evolution of a dynamical system is completely determined by its position in phase space at any given point in time. Even for high dimensional systems the dynamics is often confined to a low dimensional manifold in this space $([82])$.

Delay reconstruction is a method to obtain a representation of this manifold, if it exists, using time series recorded from the system via a process called delay embedding ([110]).

This approach has been successfully used in the cardiac sciences to separate fetal ECGs from those of the mother ([101]), and to classify different types of arrhythmias ([105]). 


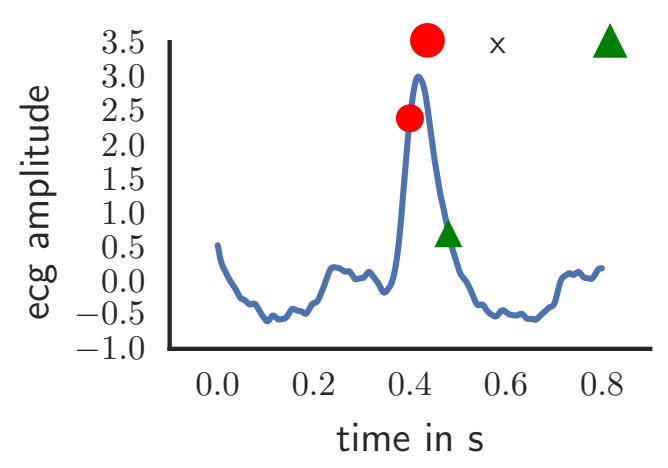

(a) Time Series

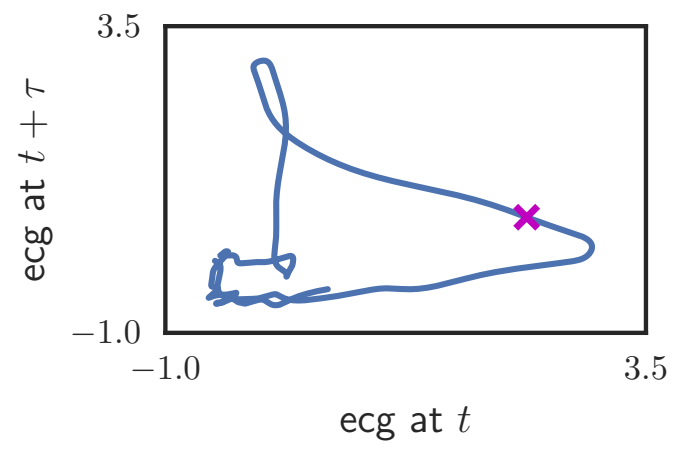

(b) Embedding

Figure 6.1.: Construction of delay vectors

a): Segment of a normal heart beat taken from an ECG recording. Two data points $0.08 \mathrm{~s}$ apart are highlighted. Using the algorithm described before, the value indicated by the circle becomes the $\mathrm{x}$ coordinate, while the one highlighted with the triangle becomes the y coordinate of a delay vector.

$b)$ : $2 \mathrm{~d}$ embedding of the ECG shown to the left using a delay of $0.08 \mathrm{~s}$. The delay vector constructed in $a$ is shown as a magenta cross. Note that in phase space trajectories can not intersect, since the position of the system in phase space does completely determine its future evolution. The embedding dimension used here must therefore be to low.

Given a time series $s(t)$ construct delay vectors as follows:

- Pick an embedding dimension $d$

- Pick a delay $\tau$

- Construct $\underline{x_{i}}=\left(s\left(t_{i}\right), s\left(t_{i}+\tau\right), \ldots, s\left(t_{i}+(d-1) \cdot \tau\right)\right)$

Takens theorem ([126]) guarantees for appropriate $d$ and under mild conditions on $\tau$ that a point set constructed in this way is a representation of the manifold on which the dynamics lives 1 . Moreover, as the delay points again form a time series one obtains a representation of the systems trajectory on this manifold. The delay reconstruction process is schematically shown in figure 6.1.

One way to condense the information contained in such reconstructed sets and trajectories is by looking at the probability that the system visits a certain subset of the phase space during its evolution. For sufficiently long time series this can be estimated from the delay trajectory, and is known to reflect its long-term behavior in phase space

\footnotetext{
${ }^{1}$ More precisely the point set is a sample drawn form a probability measure which is diffeomorph to the invariant measure of the dynamics with probability one.
} 


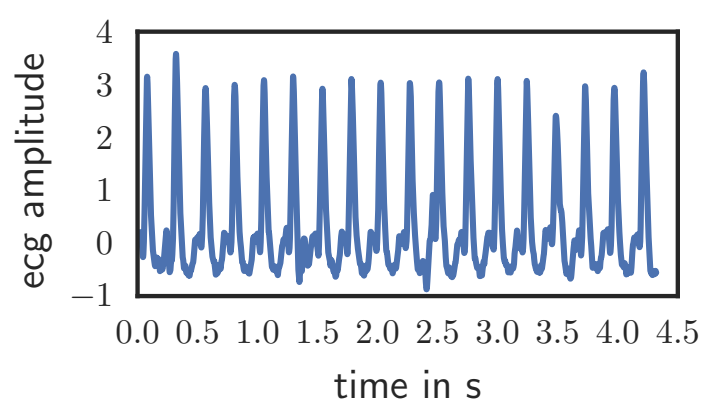

(a) ECG time series of a VT

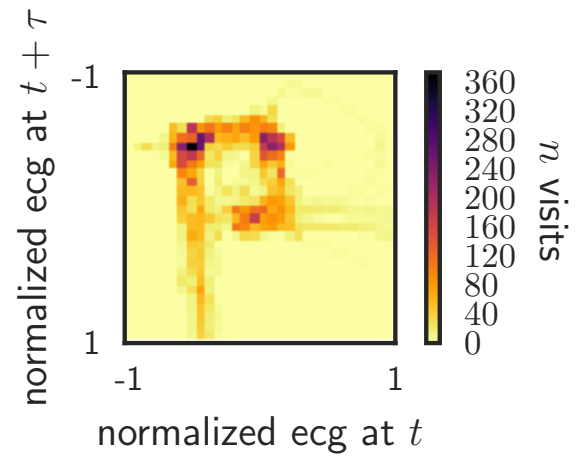

(b) Histogram of the delay embedded ECG. Embedding $d=2, \tau=0.08 \mathrm{~s}$

Figure 6.2.: Histogram of a Time Series - The time series to the right is transformed to the histogram on the left. As can be seen the system spends most of the time in a small subset of $2 d$ the delay space.

([123]). Different dynamical states of the same system can then be distinguished based on their characterizing probability distributions ([24]), which will be referred to as the delay distributions in this document.

An example of the transformation from a time series to the corresponding histogram is shown in figure 9.3 .

Several methods to pick parameters for delay embedding have been published. In this document the first zero crossing in the autocorrelation function is used as delay $\tau$, and the nearest neighbor dimension as embedding dimension $d$ (see [62] for an overview).

\subsection{Shannon Jensen Metric}

Once the histograms described in the previous section are constructed for each episode of $\mathrm{VT}$, it is possible to compare the corresponding time series using methods developed for probability densities.

[51] showed that the Kullback-Leibler divergence $D_{K L}$ is the only premetric on the space of probability distributions that fulfills a certain set of requirements. It is defined as:

$$
D_{K L}(P \| Q)=\sum_{i} P_{i} \log \left(\frac{P_{i}}{Q_{i}}\right)
$$




\section{Methods}

The individual desiderata employed in its axiomatic construction are listed below and directly linked their usefulness with respect the analysis of a time series using delay embedding. :

For probability distributions $P=\left\{p 1, \ldots, p_{i}\right\}$ and $Q=\left\{q_{1}, \ldots, q_{i}\right\}$

1. $D_{K L}$ is continuous in $P$ and $Q$

Small noise in the time series will have small effects in the computed value.

2. $D_{K L}$ is invariant under identical permutations of the labels within $P$ and $Q$ :

$$
\begin{gathered}
D_{K L}\left(\left\{p 1, \ldots, p_{i}, \ldots, p_{k}, \ldots, p_{n}\right\} \|\left\{q_{1}, \ldots, q_{i}, \ldots, q_{k}, \ldots, q_{n}\right\}\right) \\
= \\
D_{K L}\left(\left\{p 1, \ldots, p_{i}, \ldots, p_{k}, \ldots, p_{n}\right\} \|\left\{q_{1}, \ldots, q_{i}, \ldots, q_{k}, \ldots, q_{n}\right\}\right)
\end{gathered}
$$

All delay dimensions are equal, since permuting them does not influence the $D_{K L}$.

3. $D_{K L}(P \| P)=0 \forall P$ and $D_{K L} \geq 0$.

A time series is close to itself.

4. For a distribution $P=\left\{p_{i}, \ldots, p_{l}\right\}$ define $p_{i}^{*}=\frac{p_{i}}{\sum_{n=k+1}^{l} p_{n}}$ and $P^{*}=\{\underbrace{0, \ldots, 0}_{k}, p_{k+1}^{*}, \ldots, p_{l}^{*}\}$ then $D_{K L}\left(P \| P^{*}\right)$ is a monotonic increasing function of $k$.

Ignoring parts of the reconstructed delay distribution will increase the $D_{K L}$ to the original.

5. For probability distribution $P, Q, P^{0}, Q^{0}$ and conditional probabilities $\left(P \mid P^{0}\right)$ and $\left(Q \mid Q^{0}\right)$ :

$$
D_{K L}(P \| Q)=D_{K L}\left(P^{0} \| Q^{0}\right)+\mathrm{E}_{P^{0}}\left[D_{K L}\left(P\left|P^{0} \| Q\right| Q^{0}\right)\right]
$$

Where $\mathrm{E}_{P^{0}}$ is the expectation with respect to $P_{0}$.

If $P^{0}$ and $Q^{0}$ are taken to be histogram approximations of $P$ and $Q$ this guarantees that $D_{K L}\left(P^{0} \| Q^{0}\right)$ approaches $D_{K L}(P \| Q)$ in the limit of infinite data and vanishing histogram bin width. This holds as $\left(P \mid P^{0}\right)=\left(Q \mid Q^{0}\right)=1$ in this case, and $D_{K L}(1 \mid 1)=0$

Despite its desirable properties $D_{K L}$ is neither symmetric nor does it fulfill the triangle inequality ([51]). Especially the latter makes it difficult to interpret the obtained results as distances, which in turn is necessary for clustering.

The Shannon Jensen Metric was introduced in [39] to remove these short comings of the Kullback-Leibler divergence. SJM is derived by considering the mean KullbackLeibler divergence to a mixture distribution and taking the square root. It is a proper distance measure on the space of probability distributions. It fulfills both requirements 
mentioned before and retains all the given properties, albeit with respect to the mixture distribution.

$$
\begin{aligned}
M & =\frac{P+Q}{2} \\
\operatorname{SJM}(P \| Q) & =\sqrt{\left[\frac{1}{2} D_{K L}(P \| M)+\frac{1}{2} D_{K L}(Q \| M)\right]}
\end{aligned}
$$

In the context of information theory $S J M(P \| Q)$ has a beautiful interpretation: It is the Shannon information one gains by knowing that the two distributions differ (see [5] for a proof).

Given this the Shannon-Jensen metric can be viewed as measure how sensible it is to treat two empirical distributions as separate entities, since when the knowledge gain in doing so vanishes one might as well just pool the data.

\subsection{PCA on Video Data}

In addition to scalar time series our setup records four videos of the excitation on the heart surface, each one with a frame rate of $500 \mathrm{~Hz}$. To define a distance between the individual recordings a video based analysis algorithm motivated by ([143] and [139]) was implemented.

In a first step for each experiment the recorded videos are transformed into one large data matrix $\mathrm{M}$ by the following algorithm:

- Flattening each image by appending its rows to another

- Concatenating the resulting flattened images that corresponded to the same time

- Appending all flattened images of the same VT episode to each other

- Stacking the data thus obtained

As no standard mathematical notation exists for these operations, they are additionally presented in image form in figures 6.3 and 6.4 . 
6. Methods

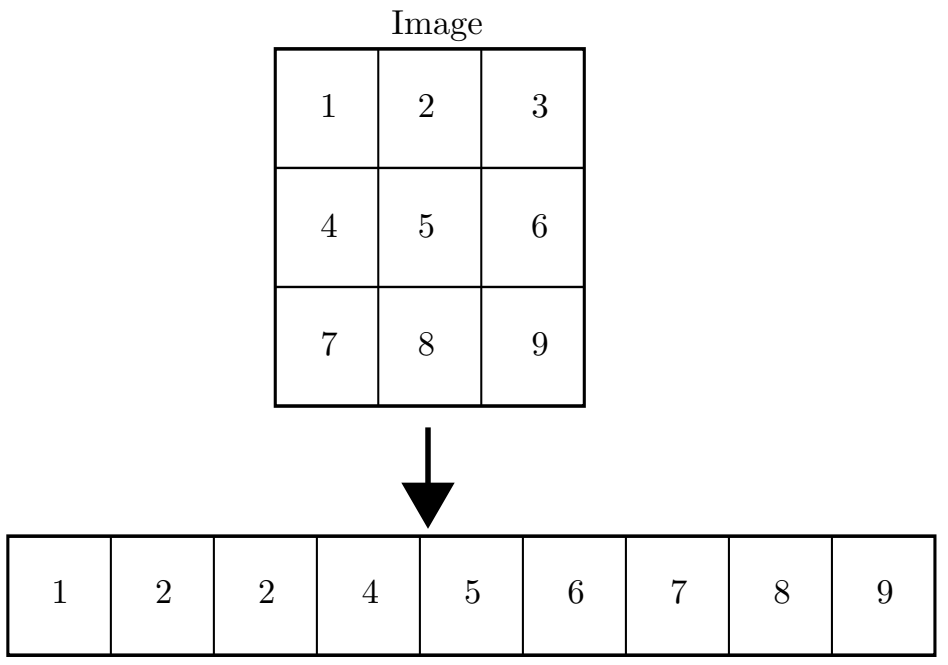

(a)

Image 0

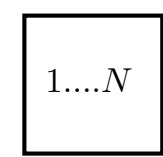

Image 1

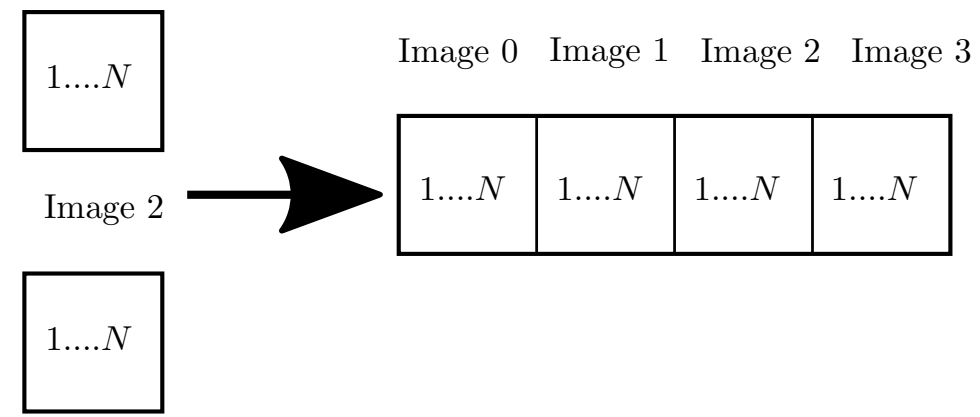

Image 3

$$
1 \ldots . N
$$

(b)

Figure 6.3.: Flattening and Concatenating Image Data - In a first step the rows of each image are appended to each other. In the second step all images corresponding to the same point in time are concatenated. 
Image 0 Image 1 Image 2 Image 3

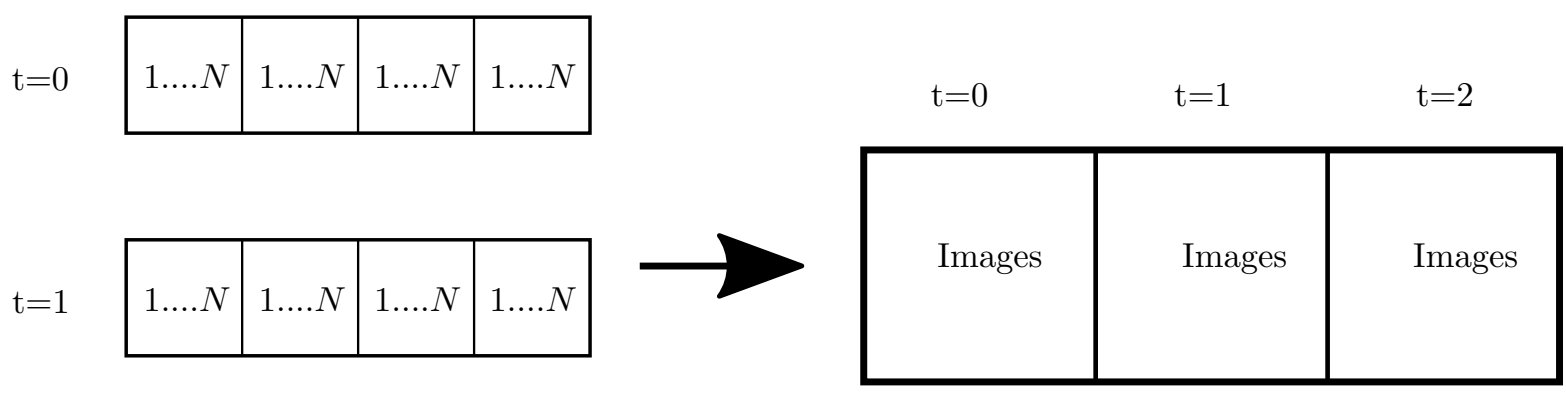

\begin{tabular}{|l|l|l|l|}
\hline $\mathrm{t}=2 \quad 1 \ldots N$ & $1 \ldots . N$ & $1 \ldots . N$ & $1 \ldots . N$ \\
\hline
\end{tabular}

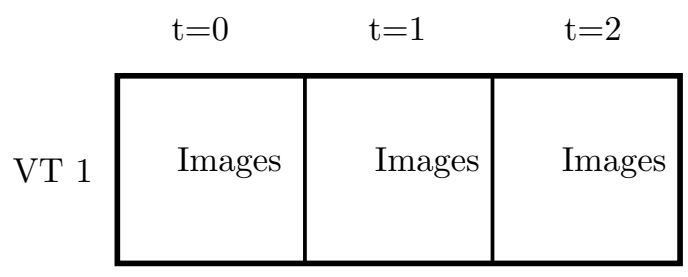

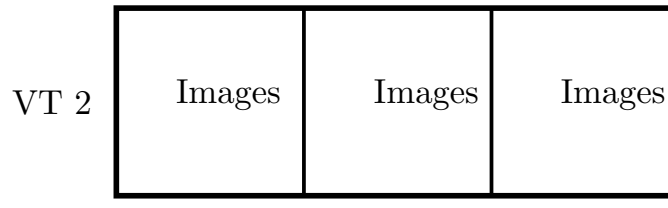

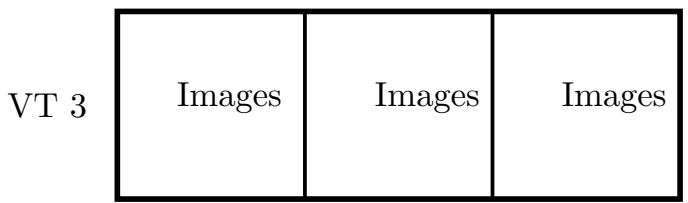

(a)

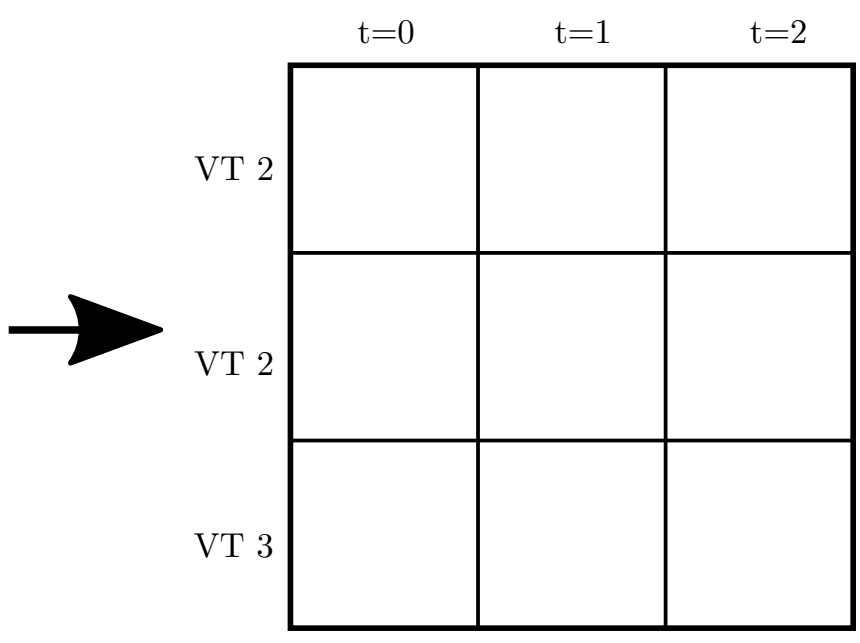

Figure 6.4.: Appending and Stacking Data - All frames belonging to the same VT episode are appended to each other. Finally all observed episodes are stacked into matrix form. The resulting matrix contains all image data obtained during the whole experiment. Dimension reduction algorithms can now be applied to that matrix.

A linear Principle Component Analysis could then be performed by solving the Eigenvalue problem for the covariance matrix $\mathrm{C}=\mathrm{M}^{T} \mathrm{M}$ associated with $\mathrm{M}$ : 
For a data matrix $\mathrm{M}$ where the ith row corresponds to the ith data vector,the principle components $V$ are defined to be the Eigenvectors of the covariance matrix $\mathrm{C}=\mathrm{M}^{T} \mathrm{M}$, i.e. those components for which:

$$
\lambda V=\mathrm{C} V
$$

Decomposing a data matrix with respect to its principle components means casting it into the basis formed by these Eigenvectors. A dimension reduction to dimension $n$ would then be performed by projecting the data matrix onto the subspace spanned by the $n$ Eigenvectors corresponding to the $n$ largest Eigenvalues.

Standard PCA methods can reduce the dimension of a data set by "ignoring" linear subspaces that carry little variance. More powerful variants of this simple PCA algorithm that can project the data onto more general subspaces can be constructed using kernel methods. For this the $l$ dimensional data vectors $\mathrm{M}_{i}$ are mapped to a $d$ dimensional space where $d>>l$ that is equipped with an inner product.

$$
\begin{aligned}
\Phi: \mathbf{R}^{l} & \rightarrow \mathbf{R}^{d} \\
\mathrm{M}_{i} & \rightarrow \Phi\left(\mathrm{M}_{i}\right)
\end{aligned}
$$

A new Eigenvalue problem is formulated in this space for a new matrix $\overline{\mathrm{C}}=\Phi(\mathrm{M})^{T} \Phi(\mathrm{M})$. The key observation is then that this eigenvalue problem can be solved knowing only the $\mathrm{M}_{i}$ and the kernel function $K\left(\Phi\left(\mathrm{M}_{i}\right), \Phi\left(\mathrm{M}_{j}\right)\right)$ that computes the inner product between the images of the data vectors (see [112] for a complete treatment).

\subsection{Spearman Correlation}

The Spearman correlation coefficient $\rho_{\text {Spear }}$ is a modification of the normal Pearson correlation. Contrary to the latter the former is defined on the ranks of the data, rather then the actual values. This makes it more suitable to compare non linearly related measures of the same effect $([79])$.

For the purpose of the computation of the $\rho_{\text {Spear }}$ the rank of a datum is defined to be its index in a sorted version of the data. A list of data points $D$ can then be transformed into a list of ranks $r_{D}$ by replacing each value with its rank. To give an example, the data list $[1,10,11,6,3,4]$ would be transformed into the rank list $[1,5,6,4,2,3]$.

The Spearman correlation coefficient between two data sets is then defined on the ranks as:

$$
\rho_{\text {Spear }}\left(D_{1}, D_{2}\right)=\frac{\operatorname{cov}\left(r_{D_{1}}, r_{D_{2}}\right)}{\sigma\left(r_{D_{1}}\right) \sigma\left(r_{D_{2}}\right)}
$$


Where $\operatorname{cov}\left(r_{D_{1}}, r_{D_{2}}\right)$ is the covariance between the ranks and $\sigma\left(r_{D}\right)$ is their respective standard deviation.

\subsection{Mantel Test}

Distance matrices, which contain the pairwise distances of points are heavily constraint. This can be understood by the following consideration: Picking $n$ points in an arbitrary metric space, the matrix of their pairwise distances has $n^{2}$ entries. Now moving a single point must change the value of $n-1$ of these entries.

Due to those constraints, normal correlations cannot be used for statistical hypothesis testing on these matrices, as a high amount of correlation originates from their definition. To solve this problem, Mantel introduced a permutation test that uses a correlation coefficient $\rho$ as test statistic $([76])$.

For two matrices under consideration first $\rho_{0}$ is computed, and then the rows and columns are randomly permuted $m$ times. For each permutation $k$ a correlation $\rho_{k}$ is computed. The p-value is then defined as the proportion of the $\rho_{k}$ that is greater than $\rho_{0}$.

In this document the Spear correlation coefficient is used as test statistic in the Mantel tests.

\subsection{DBSCAN}

Clustering is the process of automatically grouping objects based on a measure of distance in such a way that closer objects are allocated to the same group. DBSCAN ([40]) is a type of density based clustering method, which roughly speaking identifies clusters as regions of higher density that are separated by regions of lower density. The algorithm's internal definition of density and separation are controlled by two parameters $m$ and $\varepsilon$.

The algorithm operates by first identifying for each sample its neighbors, which in this case are defined as samples with a distance of less then $\varepsilon$. In the second step core samples, samples which have more than $m$ neighbors, are identified. Finally clusters are formed by recursively allocating core samples that are also neighbors to the same cluster. These cluster are then completed by adding the remaining neighbors of all core samples. 
Density based clustering algorithms posses several features that make them stand out. Firstly they do not require the cluster to have a particular shape, as opposed to centroid based clustering as e.g. K-means (see [142] for a comparison of clustering methods). Secondly they have the ability to form "noise clusters", sets of points that do not belong to any cluster ([40]). In the case of DBSCAN an example for the letter would be an isolated point with high distance to all other sample points. 
Part II.

\section{Ventricular Tachycardia}





\section{Rationale and Aims}

\subsection{Rationale}

Although not immediately life threatening Ventricular tachycardia, (VT) is a clinically relevant arrhythmia ([80]) . Even for VT in it most benign form cardioversion is recommended in current clinical guidelines $([73])$. If chemical cardioversion fails, this is done either via triggered cardioversion using a catheter guided into the heart or by external defibrillation. Both methods impose a high therapeutic burden on the patient: In case of a catheter cardioversion the patient needs to be anesthetized, which carries a risk especially for elderly individuals $([117])$; in case of defibrillation the treatment itself is painful and damaging to the heart tissue ([119]).

Reducing the field strength necessary to treat ventricular tachycardia, optimally below the human pain threshold, is therefore by itself highly relevant.

Furthermore, investigating the unpinning motivated defibrillation methods introduced in section 4.3 .2 in fibrillation is challenging. The high number of wavelets in this state leads to a possible high number of pinned waves ([133]), and the chaotic nature of the ECG during fibrillation makes it difficult to analyze using phase space methods ([45], [97]).

The phase space of ventricular tachycardia on the other hand has a much lower dimension ([105]), and is thus much more accessible using the methods from nonlinear dynamics described in chapter 6 .

As the aforementioned alternative defibrillation methods were motivated by features that VF and VT share, namely the tendency of spiral wave fragments to attach themselves to heterogeneities, low dimensional VT is a promising model system for high dimensional VF.

To investigate VT a method to reliably induce it is needed. In 4.2 it was pointed out that so far the only know anatomical correlate for stable ventricular tachycardia is mature myocardial infarction. Furthermore infarction scars are large compared to other possible heterogeneities in the tissue such as blood vessels. 
As larger obstacle emit waves at lower fields, studying VT in infarction further reduces the complexity, since it reduces the problem to that of a single spiral wave in the presence of a single virtual electrode.

However, as the induction and maturation of myocardial infarction is a procedure that induces animal suffering it is desirable to find a suitable surrogate model. Some promising alternative model systems have been proposed in the literature ([38, [111, 60] and [28]).

\subsection{Aims}

Given the considerations in the previous section, I define the following aims:

Aim 1: Model Create a model of VT due to anatomical reentry that replicates the properties described in [69] i.e:

1. The created heterogeneity acts as a wave emitting site (see 3.1 .2 )

2. VT is monomorphic

3. VT is sustained, i.e. VT lasts for more than $30 \mathrm{~s}$

4. VT is stable under shocks, i.e. cardioversion attempts do not turn the VT to $\mathrm{VF}$.

5. The same VT can be observed multiple times

The last point here will require clarification as to what the recreation of a ventricular tachycardia actually means, this will be provided in chapter 9.3.3.

Aim 2: Phase Dependence Test the phase dependence of triggered cardioversion in that model (see 3.5)

Aim 3: Phase Response Characterize the phase change in the arrhythmia due to shocks (see 3.6.1) 


\section{Experimental Methods}

Although the details of the experiments varied, the general work flow always remained the same and can be seen to the right in figure 8.1 .

Each experiment consisted of a preparation phase and an active phase. During the preparation the animal would be euthanized and the heart prepared and moved to the setup. The measuring part would then consist of a loop of arrhythmia inductions and terminations, according to the protocols specified later in the chapter.

Depending on the type of model the injury induction would either be before the euthanasia (in vivo reperfusion and 28 days infarction) or directly after the extraction of the heart (ex vivo reperfused ischemia, acute ischemia or cryoablation). The individual steps shown in this diagram are explained in the following sections in more detail.

Section 8.1 gives the details on the used euthanasia methods and cardioprotective measures, 8.2 and 8.3 continue specifying the perfusion setup, electrode configuration and data acquisition. 8.4 lines out the details of the injury induction procedures, while 8.5 and 8.6 treat the arrhythmia induction and measurement protocols. The criteria that were used to determine when to end the experiment are lined out in 8.7 and some notes on the administered drugs are given in 8.8. Finally the details for the experiments used in the analysis in 9.4 are listed in 8.9 .

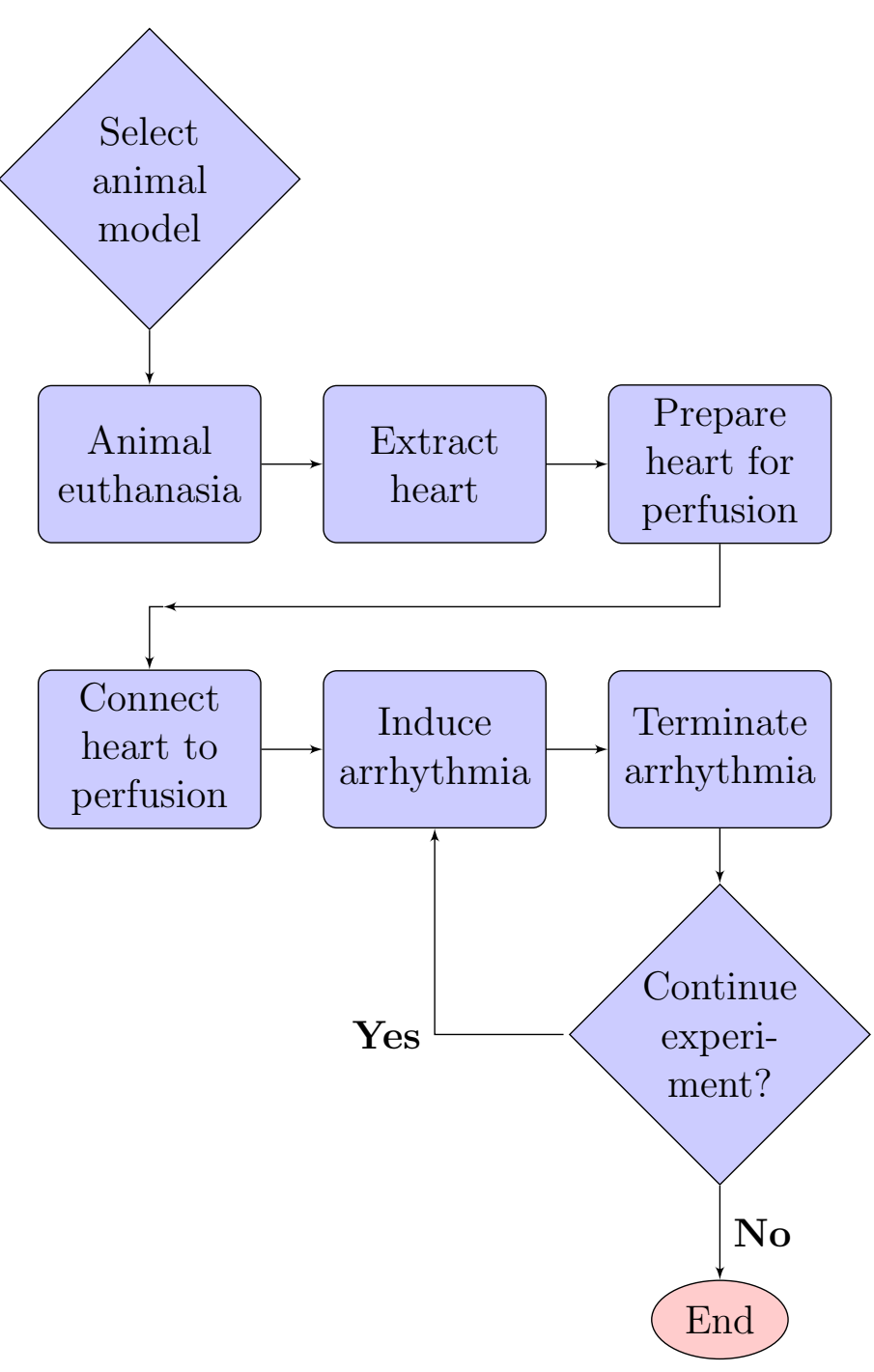

Figure 8.1.: General Workflow 


\subsection{Euthanasia and Heart Preparation}

Two types of animals were used for the experiments described in this thesis, New Zealand White rabbits $(2.5-3.5 \mathrm{~kg})$ and Göttingen mini pigs $(35-45 \mathrm{~kg})$. All animal procedures were performed in accordance with applicable local animal welfare regulations.

\subsubsection{Pig}

The animals were first anesthetized using Pentobarbital and then euthanized with $\mathrm{KCl}$ solution $\left(1 \frac{\mathrm{mmol}}{\mathrm{kg}}\right)$. Before euthanasia they were premedicated with Diazepam, and injected with heparin $\left(500 \frac{\mathrm{IU}}{\mathrm{kg}}\right)$ to avoid blood coagulation. Immediately after death, a high-potassium solution $(400-600 \mathrm{ml}, 16 \mathrm{~mm})$ as described in [18] was injected into the auricular vein to arrest the heart in a plegic state, this reduces the oxygen consumption of the cardiomyocytes, and thereby prevents ischemia. After the heart was excised it was put into $0^{\circ} \mathrm{C}$ cardioplegia solution (see table 8.1) to further reduce metabolic energy consumption.

$\begin{array}{cc}\text { Chemical } & \text { Concentration } \\ \mathrm{NaCl} & 110 \mathrm{~mm} \\ \mathrm{KCl} & 16 \mathrm{~mm} \\ \mathrm{MgCl}_{2} & 16 \mathrm{~mm} \\ \mathrm{CaCl}_{2} & 1.2 \mathrm{~mm} \\ \mathrm{NaHCl}_{2} & 10 \mathrm{~mm} \\ \mathrm{MgCl}_{2} & 16 \mathrm{~mm}\end{array}$

Table 8.1.: Cardioplegia solution

\subsubsection{Rabbit}

All rabbits were euthanized using sodium thiopental $(30 \mathrm{mg} / \mathrm{kg})$, to avoid blood coagulation they were injected with heparin $\left(500 \frac{\mathrm{IU}}{\mathrm{kg}}\right)$ directly before. The chest was opened and the heart was excised and submerged in $0^{\circ}$ cardioplegia solution identical to the procedure described for pigs. 


\subsection{Perfusion and Data Acquisition}

After extraction the plegic heart was cut free from remaining vasculature as well as fatty and connective tissue. The superfluous potassium that remained from euthanasia was washed out using cold saline solution and the heart was connected to the perfusion setup.

All hearts were perfused in the well established Langendorff method (see [32] for an in depth treatment), and as the used setup was previously described in [54], its details shall only be recalled briefly: We use a closed loop perfusion setup, the heart is submerged in warm $\left(36.8^{\circ} \mathrm{C}, \mathrm{pH} 7.3\right)$ saline solution (Tyrode for pigs and Krebs-Henseleit for Rabbits see tables 8.2 and 8.3 and retrogradely supplied with the same solution at $55 \mathrm{mmHg}$. Excess perfusate runs into a reservoir where it is reoxygenated using carbogen $\left(95 \% \mathrm{O}_{2}\right.$, $\left.5 \% \mathrm{CO}_{2}\right)$ and fed back into the perfusion system.

To make the electrical activity on the heart surface detectable the heart was stained with Di4ANEPPS ${ }^{1}([65])$, the whole cardiac surface was illuminated using LEDs (cyan $505 \mathrm{~nm}$ and green $530 \mathrm{~nm}$ ) and recorded using four EMCCD Cameras (Photometrics Evolve 128 using $610 \mathrm{~nm}$ long pass filters). To allow for automatic video analysis later on, the motion of the heart was suppressed using an excitation contraction uncoupler (Blebbistatin [116]).

In addition to the optical data two ECG-like electrical signals were recorded using two orthogonal sets of two electrodes in the bath (custom built electrodes and amplifiers). ECG and camera recordings were synchronized to allow for easy alignment of the time series. A schematic of the setup can be seen in figure 8.2 .

\footnotetext{
${ }^{1} \mathrm{~A}$ fluorescent dye that undergoes a change in its absorption / emission spectrum in reaction to changes in the membrane potential.
} 


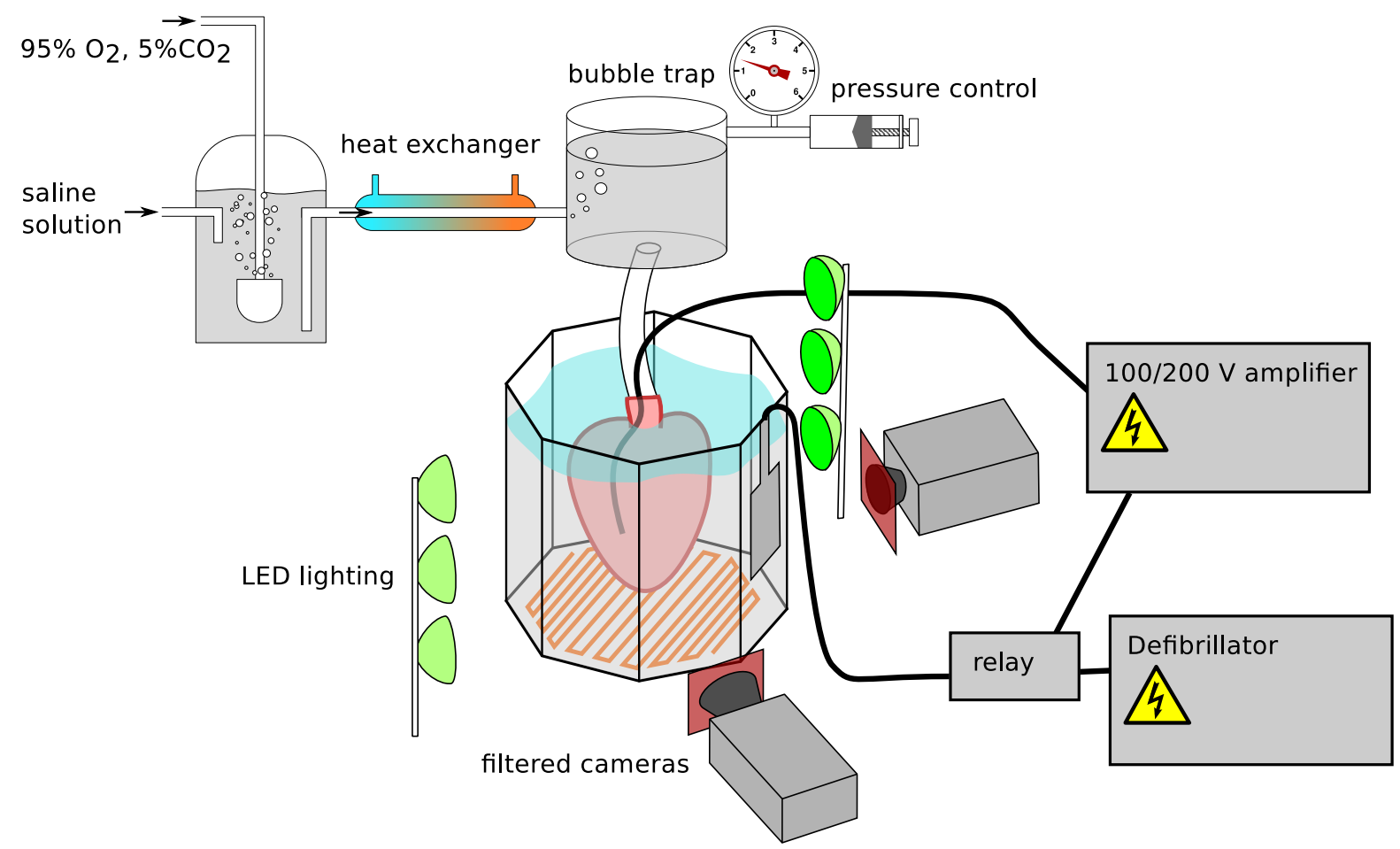

Figure 8.2.: Schematic Drawing of our Experimental Setup -

Top left: Saline solution coming from a storage container is oxygenated, heated and pumped into a bubble trap, a second closed reservoir containing solution and air.

Center: The heart is perfused by the liquid with a pressure that is dependent on the over pressure in the bubble trap. Excess liquid would run out of the perfusion bath back into the storage (not shown). To keep the hearts temperature constant the perfusion bath is heated from below. Cameras record the activation on the heart surface, which is made visible by fluorescent dyes.

Right: Shock paddle and catheter are connected to a defibrillator and a high power current source, both can be controlled via a software running on a laptop. Switching between the two is made possible via a relay.

The second stimulation electrode and the ECG electrodes have been omitted from this image.

\subsection{Electrodes}

Different electrode configurations were used for pigs and rabbits, but the same control software. All shock parameters (duration, field strength, timing) could be be controlled using this in house developed software. Furthermore it allowed to trigger the shock to a 


$\begin{array}{cc}\text { Chemical } & \text { Concentration } \\ \mathrm{NaCl} & 124 \mathrm{~mm} \\ \mathrm{NaHCO}_{2} & 24 \mathrm{mM} \\ \mathrm{NaH}_{2} \mathrm{PO}_{4} & 0.9 \mathrm{~mm} \\ \mathrm{KCl} & 4 \mathrm{~mm} \\ \mathrm{CaCl}_{2} & 2 \mathrm{~mm} \\ \mathrm{MgCl}_{2} & 0.7 \mathrm{~mm} \\ \mathrm{D}-\text { Glucose } & 0.99 \mathrm{mM}\end{array}$

Table 8.2.: Tyrode solution

$\begin{array}{cc}\text { Chemical } & \text { Concentration } \\ \mathrm{NaCl} & 118 \mathrm{~mm} \\ \mathrm{NaHCO}_{2} & 25 \mathrm{mM} \\ \mathrm{NaH}_{2} \mathrm{PO}_{4} & 1.2 \mathrm{~mm} \\ \mathrm{KCl} & 4.7 \mathrm{~mm} \\ \mathrm{CaCl}_{2} & 3.4 \mathrm{~mm} \\ \mathrm{MgCl}_{2} & 1.2 \mathrm{~mm} \\ \mathrm{D}-\text { Glucose } & 2 \mathrm{mM}\end{array}$

Table 8.3.: Krebs-Henseleit solution

previously selected phase in the ECG and apply more complicated shock protocols such as pacing from multiple electrodes or combinations of pacing and shocking.

\subsubsection{Configurations for Pigs}

An ICD catheter (Protego ProMri SD 65/18, Biotronik) was inserted into the right ventricle of the excised heart via the right atrium and screwed into the right ventricular endocardium. The myocard could be stimulated locally with a pacing electrode at the tip of this catheter, while a coil $16 \mathrm{~mm}$ distal from the tip was used as the anode for shocking. A plate electrode $(35 \mathrm{~mm} \times 50 \mathrm{~mm})$ that was attached to the perfusion bath was used as cathode. A second pacing electrode was inserted into the epicardium directly below the right atrium, to allow for more complicated stimulation protocols.

For the investigation of phase dependence of cardioversion, cathode and anode were connected to a high precision, high power current source (BOP 1KW-MG,Kepco). Normal defibrillation was performed using a different previously described defibrillator ([54]) via the same electrodes; switching between the high precision current source and the defibrillator was possible with a relay.

\subsubsection{Configuration for Rabbits}

As rabbit hearts are too small to use catheters, two plate electrodes were used that were attached to opposite sites of the perfusion bath. Theses electrodes were connected to the same programmatic controlled high precision, high power current source described previously, but as the used bath was much smaller no additional defibrillator was needed. Contrary to pigs only a single pacing electrode was placed on the epicardium for rabbits. 


\subsection{Injury Induction}

\subsubsection{Cryoablation}

Cryo injuries were applied using hex keys that were cooled using liquid nitrogen. The exact procedure was modified several times during the experiment run in an attempt to modify it in such a was as to obtain a model that fulfilled the desiderata listed in section 7.2 or to overcome observed problems.

In total four different cryoablation procedures were used, that differed in the number of freeze-thaw cycles of the injured areas as well as the number and placement of the injury sites. The different procedures are listed below:

O A single cryoablation, applied apically on the left ventricle using an $8 \mathrm{~mm}$ hex key. Only a single freeze-thaw cycle.

I A single cryoablation, applied apically on the left ventricle using a $10 \mathrm{~mm}$ hex key. Only a single freeze-thaw cycle.

II Two cryoablations directly next to each other, applied apically using an $8 \mathrm{~mm}$ hex key. After inspection of the optical signal, sites that showed activity after the tissue recovered from freezing were ablated a second time.

III Three cryoablation sites directly next to each other, applied apically on the left ventricle using an $8 \mathrm{~mm}$ hex key. Two to three freeze-thaw cycles.

IV Two cryoablation sites, applied next to each other using an $8 \mathrm{~mm}$ hex key. A small isthmus was left between the sites to allow the possibility of reentry. One to two freeze-thaw cycles if deemed necessary based on the optical signal.

\subsubsection{Ischemia in Rabbits}

Ischemia in rabbit hearts was induced by ligating the left coronary artery directly below its second branching point using surgical suture and a surgeons knot. To increase the pressure on the blood vessel a small peace of tubing was put under the knot. This ligation was left in place for 30 minutes, afterwards the hearts were allowed to recover for 30-60 minutes. 


\subsubsection{Ischemia in Pigs}

Reperfused ischemia in pigs was induced using a surgical intervention on a living animal by a veterinarian, the surgical method has been described in [91. To summarize it briefly: A small insertion was made near the clavia and an inflatable catheter was inserted into the subclavian artery. This catheter was moved along the artery to the aorta and then guided into the second branch of the left anterior descending coronary artery. The balloon was inflated, thereby inducing acute ischemia in the tissue that was supplied by this branch. After 90 minutes the catheter was removed, allowing reperfusion of the ischemic tissue. The animal was then sacrificed and the heart moved to the perfusion setup.

As it will be of importance later the anesthesia and analgesia protocol shall be described here in some detail: Before the surgery the animals received $10 \mathrm{mg}$ Ketamin and $2 \mathrm{mg}$ Azaperon, an anesthetic and a sedative respectively. During the procedure the animals were narcotized with 20-60 mg Propofol, $100 \mu \mathrm{g}$ Fentanyl were injected as analgesic. Both drugs were in addition continuously applied at $8 \frac{\mathrm{mg}}{\mathrm{kgh}}$ and $5-10 \frac{\mathrm{\mu g}}{\mathrm{kgh}}$ respectively.

Acute ischemia in pigs hearts was induced using the same method described for rabbits.

\subsubsection{Myocardial Infarction}

The same injury induction procedure described for reperfused ischemia 8.4 .2 was performed to induce myocardial infarction, however in this case the animal was not sacrificed after reperfusion but the infarct was allowed to mature for 28 days afterwards.

\subsection{VT Induction}

In all non infarction hearts VT induction was attempted using either a series of 3-20 fast local stimuli via either one of the pacing electrode or using 3-4 far field shocks $\left(0.5-2 \frac{\mathrm{V}}{\mathrm{cm}}\right)$ followed by a single stronger shock (1.5-2.5 $\left.\frac{\mathrm{V}}{\mathrm{cm}}\right)$ after 100-200 ms (see [104]).

In the infarcted hearts additionally a clinical induction protocol was used that prescribed first a series of 10 pulses from the catheter stimulus electrode followed by up to 3 fast additional stimuli from the second pacing electrode near the atrium (see [80, table 1111]). 


\subsection{Measurement protocols}

Two different measurement protocols have been used:

Protocol 1 The general idea behind this protocol was that if cardioversion success was phase dependent, then the minimum field strength necessary to terminate VT should be different for different phases.

- Classify VT morphology by hand from the ECG

- For each morphology

- For each of 5 phases $\phi_{i}$, which are equally distributed over the unit cycle:

- Apply monophonic shocks of increasing strength at $\phi_{i}$ until the arrhythmia is terminated

[104] used an identical protocol in their investigation, however in my case it proved insufficient. Data collected in this way could not be analyzed if the hand classification done in the first step was wrong. See figure $8.3 \mathrm{a}$ for an illustrative example how data obtained via this measurement protocol would look like, assuming a phase dependence identical to the one reported in [104].

Protocol 2 To overcome the short comings of protocol 1, protocol 2 was designed.

- Select shock strength

- Apply at least 5 separate monophonic shocks at random phases

- If VT turns to VF due to shock: Decrease field strength.

- If VT cannot be terminated: Increase field strength.

- Classify morphologies after the experiment

The difference between the two protocols is illustrated in figure 8.3 


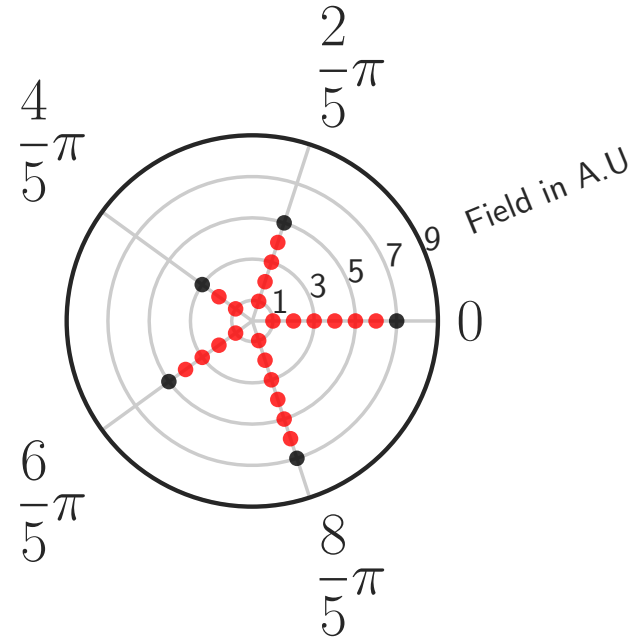

Phase

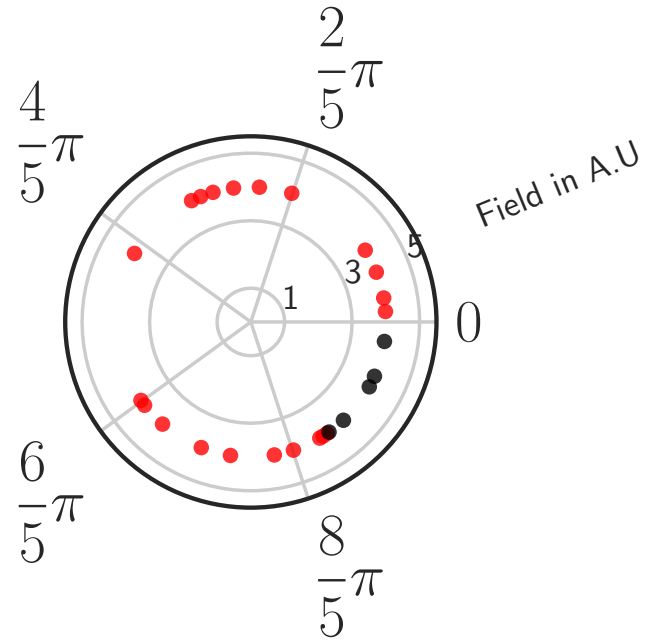

Phase

(a)

(b)

Figure 8.3.: Mock Data for Protocol 1 and 2 - Schematic representation of data that the protocols would generate. Red dots symbolize unsuccessful cardioversions, black dots successful ones.

a) A possible phase dependence would be detected using this protocol by the different radial extends of the measurements at the different phases. However, this is sensitive to misclassifications: If of the 26 data points the two successful events at $\frac{2}{5} \pi$ and $\frac{4}{5} \pi$ were removed (for example because in a post experiment analysis it would turn out that they belong to a different VT morphology) no statement could be made anymore for the want of data. b) As the points are randomly distributed over the phase, this protocol is much less sensitive to the removal of single data points. Deleting any two of the successful terminations would still allow to detect phase dependence.

\subsection{Criteria to End the Experiment}

The experiments were terminated if one of the following criteria was met:

- Repeated spontaneous fibrillation

- Global ischemia, visible in optically recorded action potentials

- Inability to defibrillate

- Strong drift of the defibrillation threshold 


\subsection{Drugs}

As the aim of the conducted experiments was the induction of stable monomorphic ventricular tachycardia two different antiarrhythmic agents have been used in an attempt to further this goal.

Flecainide acetate Flecainide is a class Ic antiarrhythmic agent that causes rate dependent channel block in the Nav1.5 sodium channel and thereby causes the conduction velocity to decrease and the wavelength to increase ([28]). It has been shown to be effective in the induction of monomorphic ventricular tachycardia in rabbits ([104]), and dogs $([69])$, however in both cases in conjunction with myocardial infarction.

Ajmaline Ajmaline is a class Ia antiarrhythmic agent that acts on both the cardiac sodium and potassium channels ([43]), thereby prolonging the cardiac action potential. It has been shown to cause tachycardia in the ischemic heart ([11]]).

\subsection{Details for the experiments}

The sheer number of performed experiments and modified experimental parameters makes it impossible to discuss all of them, so here the scope will be limited to the experiments that were used in the analyses performed in 9.4 , a summary can be seen in table 9.4 .

One of the biggest differences between the experiments is the field strength of the applied shocks. Partly this is due to differences in the inherent properties of the hearts themselves: While it might possible to observe a certain effect using a particular field in one heart, another organ may require a much stronger shock to show the same behavior. This type of behavior is well documented for defibrillation ([86]). A second source for the differences between the used fields are the two different used protocols. In particular protocol 1 lead to shocks at widely different fields in the experiments. This can be seen in figure 8.4, the three rabbit experiments that were performed using protocol 1 have much broader distributions of applied field strengths then it is the case in the pig experiments. 


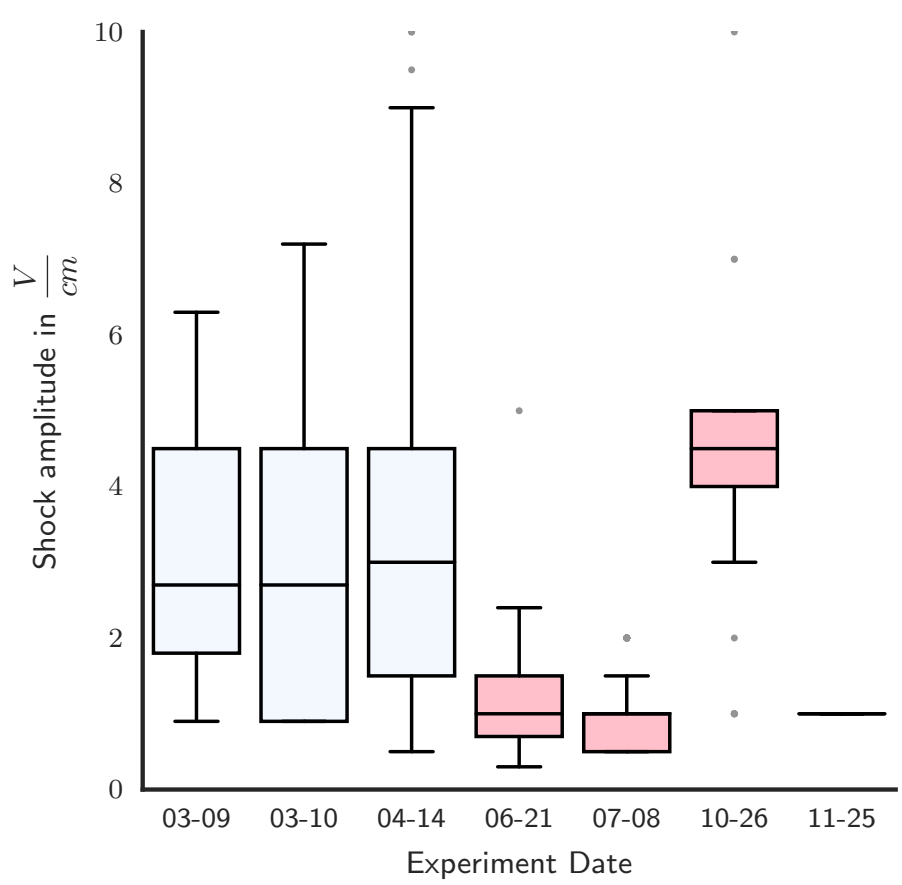

Figure 8.4.: Box plot of the shock field strengths of the experiments - The central line indicates the data median, upper and lower bound of the box are the quartiles, whiskers extend to quartile plus 1.5 time the inter quartile range. Points outside the range covered by the whiskers are drawn separately. Experiments on pigs are colored pink, experiments on rabbits light blue. For the rabbit experiment on 2016-11-25 only one field strength was used, it is indicated by the black bar. 


\begin{tabular}{|c|c|c|c|c|c|c|}
\hline Date & Injury Type & Animal & Blebbistatin & Drugs & Protocol & Notes \\
\hline 2016-03-09 & Cryo (IV) & Rabbit & $(+-) 5.8 \mu \mathrm{M}$ & Flecainide $1.5 \mu \mathrm{M}$ & 1 & $\begin{array}{l}\text { The heart was perfused at } \\
40 \mathrm{mmHg} \text {, so slightly below the } \\
\text { normal perfusion pressure }\end{array}$ \\
\hline 2016-03-10 & Cryo (IV) & Rabbit & $(+-) 3.2 \mu \mathrm{M}$ & Flecainide $1.5 \mu \mathrm{M}$ & 1 & $\begin{array}{l}\text { As on the day before the heart } \\
\text { was perfused at } 40 \mathrm{mmHg}\end{array}$ \\
\hline 2016-04-14 & Cryo (II) & Rabbit & $(+-) 2.4 \mu \mathrm{M}$ & Flecainide $1.5 \mu \mathrm{M}$ & 1 & Again perfused at $40 \mathrm{mmHg}$ \\
\hline 2016-06-21 & infarction pig & Pig & $(+-) 5.0 \mu \mathrm{M}$ & None & 2 & $\begin{array}{l}\text { Before the heart was extracted it } \\
\text { was used for an in vivo exper- } \\
\text { iment. During this experiment } \\
\text { the animal received Propofol and } \\
\text { Fentanyl. }\end{array}$ \\
\hline 2016-07-08 & uninjured & Pig & (-) $1.2 \mu \mathrm{M}$ & None & 2 & \\
\hline $2016-10-26$ & acute ischemia & Pig & (-) $2.3 \mu \mathrm{M}$ & Ajmaline $0.66 \mathrm{~nm}$ & 2 & \\
\hline $2016-11-25$ & Cryo (IV) & Rabbit & (-) $2.3 \mu \mathrm{M}$ & None & 2 & \\
\hline
\end{tabular}

Table 8.5.: Summary of the experiments -

First column: Date of the experiment

Second column: Injury induction method as outlined in 8.4

Third column: Species of the animal

Fourth column: Concentration of Blebbistatin in the perfusion liquid.

(+-) denotes an even mixture of the two possible stereo eniantomeres, (-) denotes the pure bio-active chemical.

Fifth column: Concentration of additional drugs in the perfusion liquid

Sixth column: Additional comments regarding the experiment 


\section{Experimental Results}

\subsection{Overview}

In total 26 experiments were performed with the goal to induce VT. Two additional experiments that showed spontaneous VT were provided by Henrik Tom Wörden and Dr. Daniel Hornung. In nine more experiments VT induction was attempted, although it was not the main goal.

Three different surrogate models for myocardial infraction and one model of mature myocardial infraction were investigated in two different species:

- ischemia with reperfusion in pigs (reperfused with blood)

- Cryoablation in rabbits

- Acute ischemia in pigs

- Acute ischemia with reperfusion injury (reperfused with Tyrode Solution)

- 28 day old mature myocardial infarction in pigs

Monomorphic VT episodes that lasted for more than 30 seconds could be repeatedly induced in seven of these hearts, sporadic VT could be observed in almost all hearts. Table 9.1 gives an overview of the performed experiments. Although wave emission could not be inspected visually for all experiments due to varying image quality, it could be confirmed that artificially created heterogeneities acted as wave emitting sites for all models. In the ex vivo reperfused ischemia experiments the wave emission seemed to diminish over time. This is consistent with [25] where it was observed that gap junction uncoupling which is responsible for the change in intra cellular conductivity is reversible.

The high number of experiments is due to an ongoing attempt to modify experimental parameters in such a way as to find a model that fulfills all desiderata outlined in 7.2 . The results of this attempt will be discussed in section 9.5 . 


\begin{tabular}{|c|c|c|c|}
\hline Animal & Type & $N$ & \# Repeated VT \\
\hline \multirow[t]{4}{*}{ Pig } & acute ischemia & 1 & 1 \\
\hline & in vivo reperfused ischemia & 9 & 0 \\
\hline & chronic infarction & 6 & 1 \\
\hline & spontaneous & 1 & 1 \\
\hline \multirow[t]{6}{*}{ Rabbit } & Cryo (O) & 7 & 0 \\
\hline & Cryo (I) & 2 & 0 \\
\hline & Cryo (II) & 1 & 1 \\
\hline & Cryo (III) & 1 & 0 \\
\hline & Cryo (IV) & 4 & 3 \\
\hline & ex vivo reperfused ischemia & 5 & 0 \\
\hline
\end{tabular}

Table 9.1.: Overview of all performed experiments Column one: Animal model Column two: Injury type (see chapter 5) Column three: Number of experiments Column four: Number of experiments that allowed for the repeated induction of sustained metamorphic VT

As a consequence of this repeated modification, the successful experiments differed in their injury induction procedures and measurement protocols (see figure 9.4 for a summary of the shock field strengths in the experiments). Data from different experiments could therefore not be pooled and all further analysis was performed for each experiment separately.

Within each experiment tachycardia episodes before and after shock were considered to be distinct; a successful induced VT that could be shocked five times before termination was therefore treated as five different episodes. This is necessary as VT morphologies sometimes change as the result of electrical field stimuli.

To analyze the collected data with respect to the aims set in 7.2 , first a method to quantify how similar VT episodes are is developed and tested (section 9.3). Using this method the stability (section 9.4.1) and reproducibility (section 9.4.2) of VT in the different experiments is then accessed. Once this is done the different models are compared. Finally, phase transition and phase dependence of cardioversion are investigated in the single experiment satisfying the requirements of reproducibility and stability. 


\subsection{Grouping VT}

At this point the reader is encouraged to recall that of the two used measurement protocols, protocol one suffered form the problem that tachycardias were wrongly hand classified into morphologies. This led t a disfavorable sampling of the phases of the particular morphologies (see section 8.6 for more details and an graphical explanation).

These problems motivated the development of a method to systematically aggregate the individual tachycardia episodes into groups. Different methods to do this have been used in the past: In a clinical setting, where optical mapping is of course impossible, this is still mostly done manually by cardiologists reviewing ECG recordings and entire volumes of books are devoted to ECG interpretation ([80]). The reasoning here is: As the ECG is a deterministic, continuous function of the excitation in the heart muscle, similar excitation patterns will lead to similar ECG traces. In the case of VT this means that similar located spirals can be grouped via the ECG without knowledge about their actual location.

One example of the application of this method to laboratory science can be found in [69] where it was used to group multiple instances of VT manually by identifying similar patterns in the ECG.

An alternative to manual grouping is machine learning. A rich literature that deals with its applications to the problem exists, but it is concerned with correctly identifying different types of arrhythmic states ([141], [58], [87]) or optimizing the shock timing ([21], [115]), rather than finding structure within different instances of the same arrhythmia in the same individual.

Nevertheless a method for classification without direct human intervention, preferably from the ECG alone is desirable: Firstly, the statistical significance of results will be extremely sensitive to the grouping (see e.g. [136] or [78] ). Eliminating the human factor guards against systematic experimental errors.

Secondly, possible translations to clinical applications will need to be implementable in hardware and rely solely on the ECG.

Finally and maybe most important, ventricular tachycardia may again serve as an simpler model for fibrillation (see 7.1): In [10] we observed intermittent phases of lower complexity in VF, one of the motivations of this research was the VT like appearance of this episodes in the ECG. If it is possible to distinguish different tachycardic states from a time series alone, this method might be extendable to these low complexity states we observed in fibrillation. 


\subsection{Distance between VT episodes}

The algorithm used to first create distance matrices and then cluster the tachycardia episodes on experiment level can be summarized as follows:

\section{For each experiment}

\section{For each VT:}

- Apply delay reconstruction to the ECG (see 6.1)

- Approximate the characterizing probability distribution by a histogram over the reconstructed points (see 6.1)

- Compute a pairwise distance measure between histograms

- Perform clustering using these distances

These points will be described in the following in more detail.

\subsubsection{Delay Reconstruction from the ECG}

For each VT episode two seconds of ECG before the shock were extracted. For all ECGs the SNR was high and remaining noise could be removed using a Butterworth filter. From the thus obtained time series the mean was subtracted and it was scaled to be in the range between minus one and one. The embedding dimension was estimated using the false nearest neighbor algorithm ([62]) for each episode, and the maximum needed dimension was used to embed all VTs.

For all episodes the first zero in the autocorrelation function was computed and the $75 \%$ percentile of the distribution of this value within an experiment was used as embedding delay for time series from that experiment. Using the same delay for all time series that are to be compared is necessary to make the reconstructed sets comparable ([110]). To validate this value all computations were carried out for 10 different equally spaced delays in the interval between the $20 \%$ and $80 \%$ percentiles; the obtained similarity matrices were all significantly correlated according to the mantel test (see 6.5).

The reconstruction process can again be described very compact in algorithm form:

\section{For each VT}


- Extract and filter the ECG

- Subtract the mean and scale

- Compute $\tau_{t}$ the first zero crossing of the auto correlation

- Compute $d_{t}$ the false nearest neighbor dimension

\section{For each VT}

- Embed the time series using the delay $\tau=\max \left(\left\{\tau_{t}\right\}\right)$ in dimension $d=$ $\max \left(\left\{d_{t}\right\}\right)$

\subsubsection{Histogramming}

All observed VTs in all experiments had a nearest neighbor dimension between three and five, hence all used histograms are five dimensional. To further facilitate histogram comparison the bins were chosen to be uniform in the interval between -1 and 1 , and as there are no preferred dimensions in delay space all dimensions were binned equally. Different numbers of bins were tested on a subset of the data, no qualitative differences could be observed from 7 to 15 bins per dimension, the computed distance differed by less than $10 \%$. Replacing the histograms with probabilities computed from a kernel density estimator trained on the data did likewise not change the qualitative results. For the further analysis histograms with 10 bins per dimension were chosen as compromise between needed computer memory and resolution.

\subsubsection{Measuring Similarity}

In section 6.2 it was mentioned that the Shannon-Jensen Metric can be viewed as a measure of how well the data that give rise to tow empirical distributions can be pooled. Since the ultimate goal of establishing a distance relation between VTs is to use it for clustering, which is at its core nothing but an automatized way to decide which data to pool, SJM is an inherently sensible choice. Hence it was used as distance measure between the normalized histograms (see 9.1), and the pairwise distances were stored in a distance matrix. Row and column indices correspond to the running number of the observed VT episode: $d(i, j)$ therefore contains the SJM distance between the $i t h$ and the $j$ th observed tachycardia.

An example can be seen in figure 9.1. Black ticks on the $\mathrm{x}$ axis indicate where an arrhythmia episode ended, either by transformation to VF or termination. The heart 


\section{Experimental Results}

was allowed to recover for three minutes at these points and a new tachycardia episode was induced. Consequently $n$ is not completely equivalent to experiment run-time, although episodes with adjacent $n$ are closer in time than episode with high difference in their running number. A change in the arrhythmia without reinduction can be seen between $n=20$ and $n=30$, events like this make it necessary to consider episodes before and after a shock as separately.

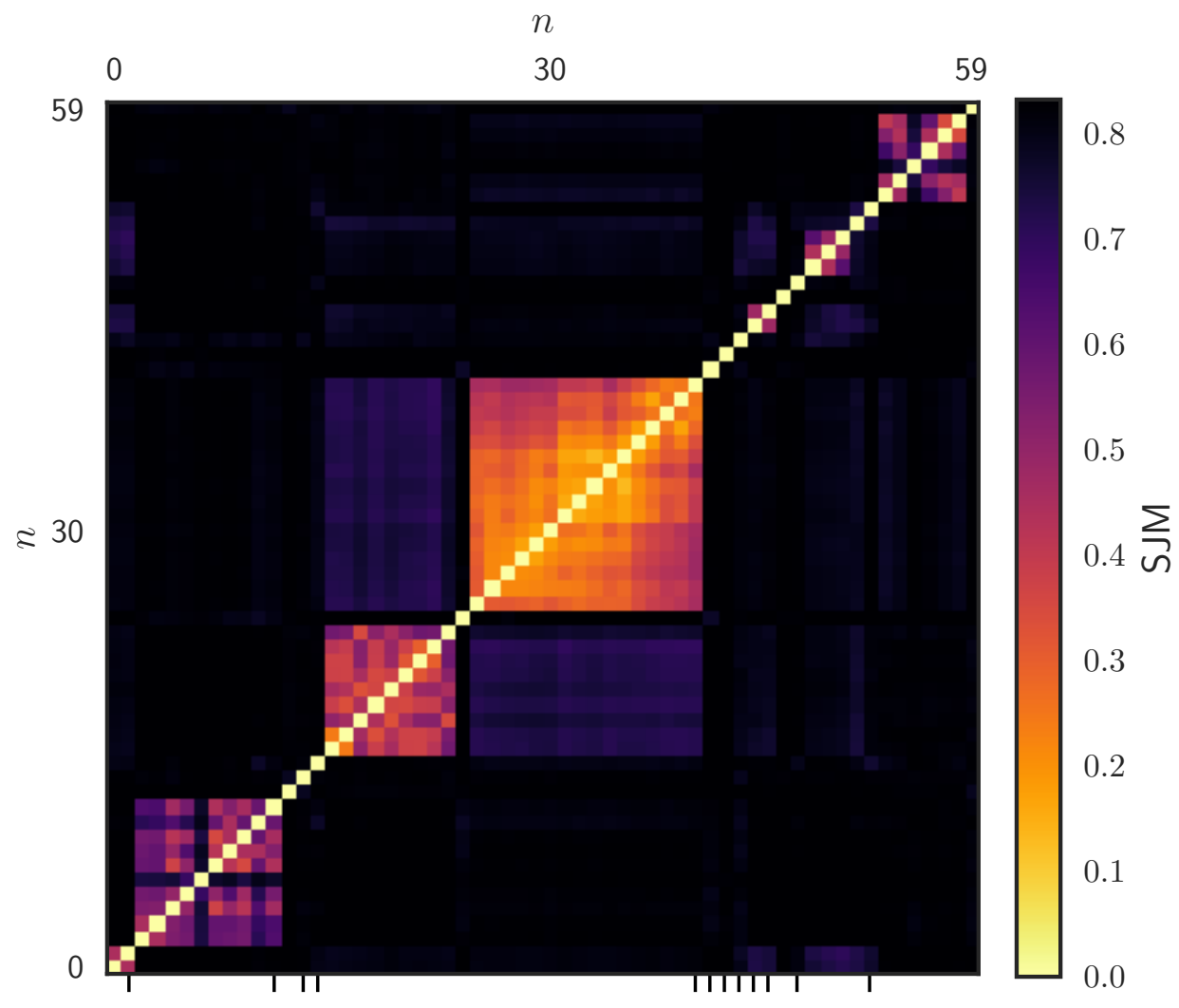

Figure 9.1.: SJM distance matrix based on the experiment performed on 2016-10-26 $n$ is the running number of the VT episode, black ticks on the $\mathrm{x}$ axis indicate a pause of 3 minutes and reinduction of a fresh tachycardia.

Using either the Hellinger distance or the $L 2$-norm as distance between the reconstructed histograms resulted in matrices that were strongly correlated ${ }^{1}$ with the Shannon-Jenson matrix.

\footnotetext{
${ }^{1} p<0.005$ using the mantel test
} 


\subsubsection{Clustering}

Once distance metrics are computed different clustering algorithms can be used to group the VTs, but care has to be taken that the underlying assumptions of the algorithm are not in conflict with the data structure.

As the Langendorff perfused heart is a non stationary system due to the fact that the organ is slowly dying, ECG segments taken at different times show differences even if the overall dynamics is similar. Observations of the same tachycardic state made close in time should therefore also be closer according to the used distance measure. The cluster structure one would expect in this case is similar to smeared out blobs: While elements within the blobs are close to their neighbors they can still be quite distant from other members of the same blob. At the same time the blobs themselves are clearly separated.

Cluster structures like the one described above are well detectable using density based clustering where clusters are defined to be regions of higher density separated by regions of low density (see 6.6 for more details), and consequently in the remainder of this document the DBSCAN algorithm ([40]) was used to detect clusters.

\subsubsection{Similarity Measure Evaluation}

Two additional types of distance matrices were computed and compared with the ones based on delay reconstruction.

\section{Maximum Correlation}

For each pair of VTs the maximum of the normalized crosscorrelation function was computed between the ECG segments that were previously used for the delay reconstruction. A distance matrix can then be defined using:

$$
d_{\text {correlation }}(X, Y)=1-\rho(X, Y)
$$

Where $\rho$ is the aforementioned maximum normalized crosscorrelation.

Visual inspection of the obtained matrices showed that maximum correlation obtained similar structures as the delay reconstruction based method in cases where the grouping was very pronounced (e.g. 9.2d) but was more diffuse in cases where the delay method failed to show clear proximities (e.g. 9.2a). Inspection of the videos taken at 2016-0414 and 2016-11-25 revealed that the maximum cross correlation assigned low distances to tachycardias that had visually different expiation patterns, but similar dominant 


\section{Experimental Results}

frequencies. In addition maximum correlation seems to be less fine grained, of its possible range effectively only the interval from 0.5 to 1 is used. Exemplary distance matrices for two days can be seen in figure 9.2 .

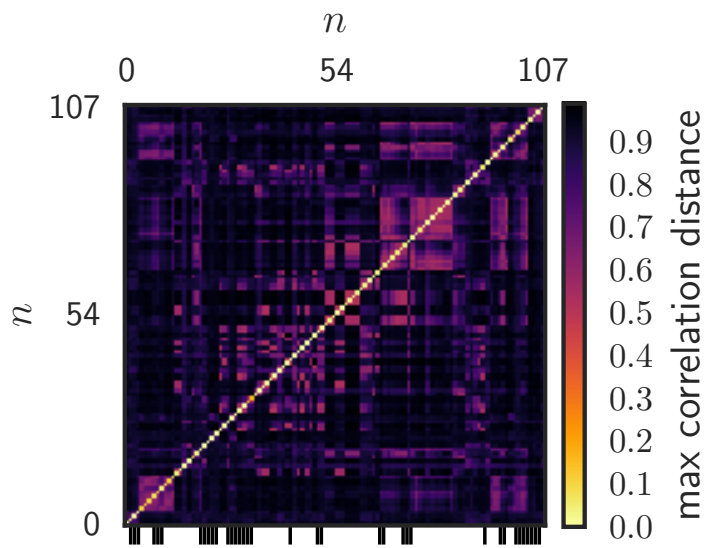

(a) Correlation distance for the experiment performed on 14-04-2016

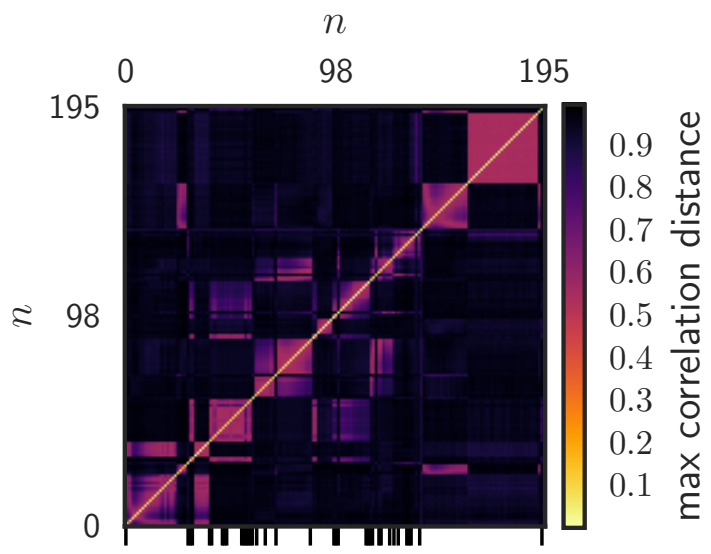

(c) Correlation distance for the experiment performed on 21-06-2016

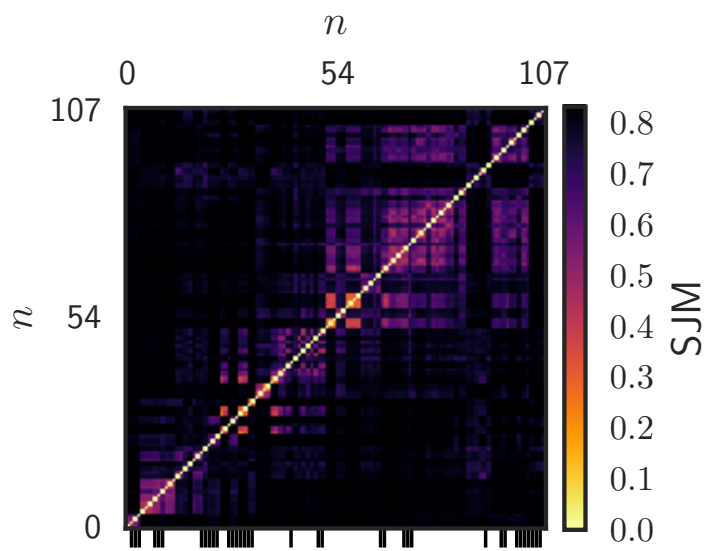

(b) SJM distance for the experiment performed on 14-04-2016

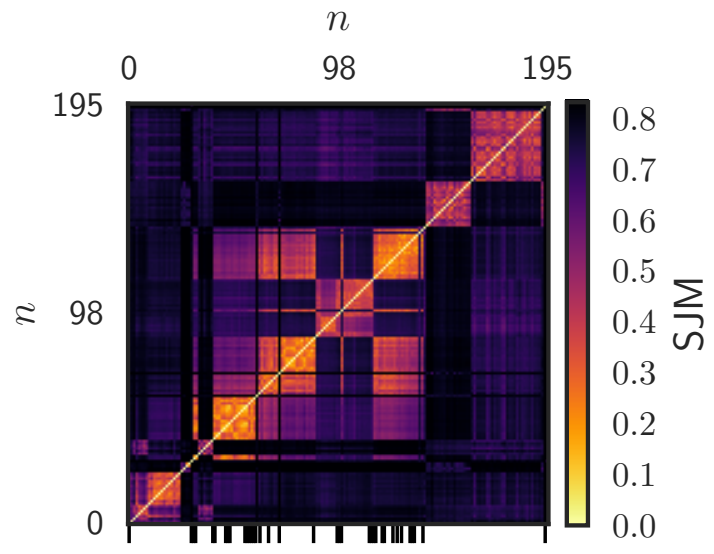

(d) SJM distance for the experiment performed on 21-06-2016

Figure 9.2.: Pairwise distances between observed VTs using two different methods $n$ is the running number of the observed VT and is therefore correlated with time.

a)-b): The block like structure along the diagonal visible in b) can also be seen in $a$ ), but in addition the correlation assigns low distances to elements farther away from the diagonal, resulting in a less coherent pattern.

c)-d): Similar patterns along the diagonal axis are visible in both images. For entries farther away from the diagonal the two methods differ more strongly. 
Video PCA Based Distance

Analysis of video data was possible for three of the seven experiments, in the other four cases image quality was too low to allow processing. Video segments that corresponded to the previously used ECGs were extracted from the recordings of the four synchronized cameras of our setup. These videos were then aligned on the first maximum in the ECG and a dimension reduction via a kernel PCA using a radial kernel was performed (see section 6.3 and scikit-learn [89] for the implementation). Pairwise euclidean distances were then computed between the projections of the videos on the PCA components that accounted for $80 \%$ of the variance. For all analyzed videos the number of these components was below $10 \%$ of the number of data points, which indicates that the data sets can be decomposed quite well using this method. 


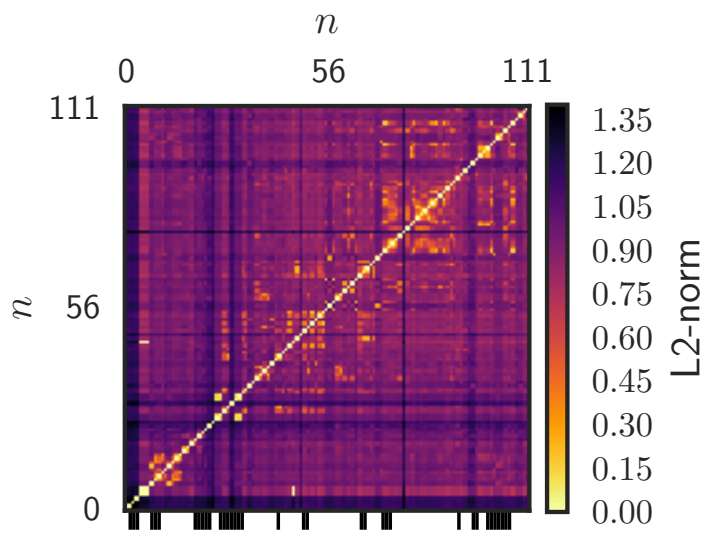

(a) Video data base distance for the experiment performed on 14-04-2016

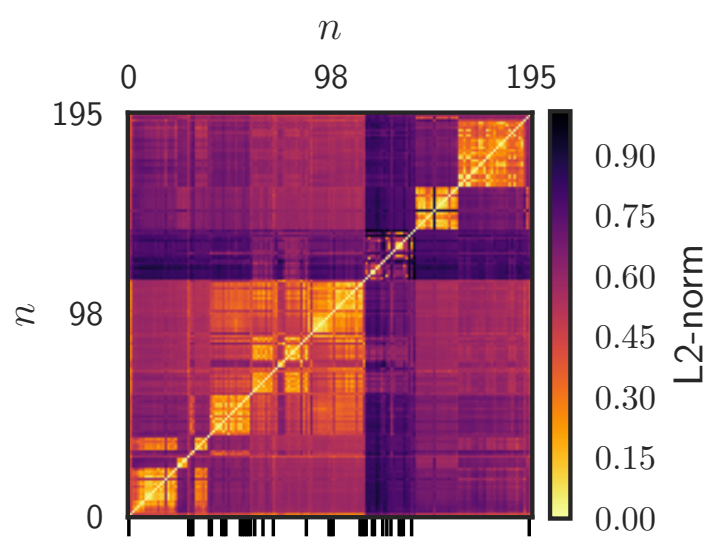

(c) Video data base distance for performed on 21-06-2016

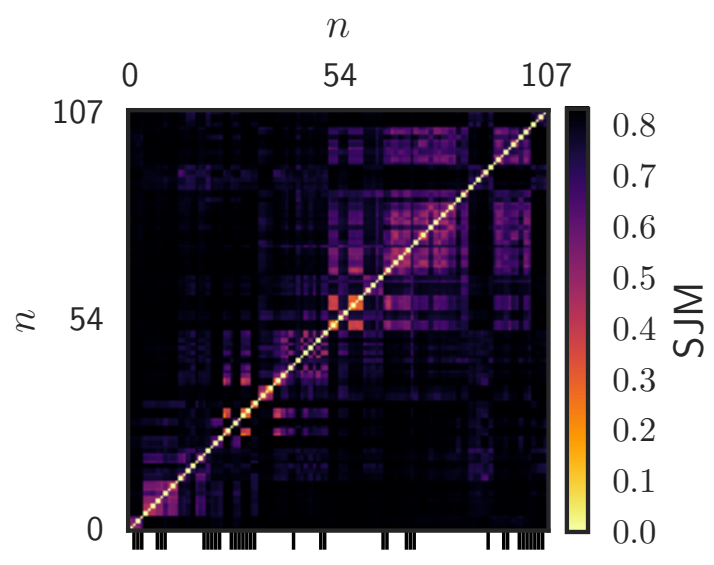

(b) SJM distance for the experiment performed on 14-04-2016

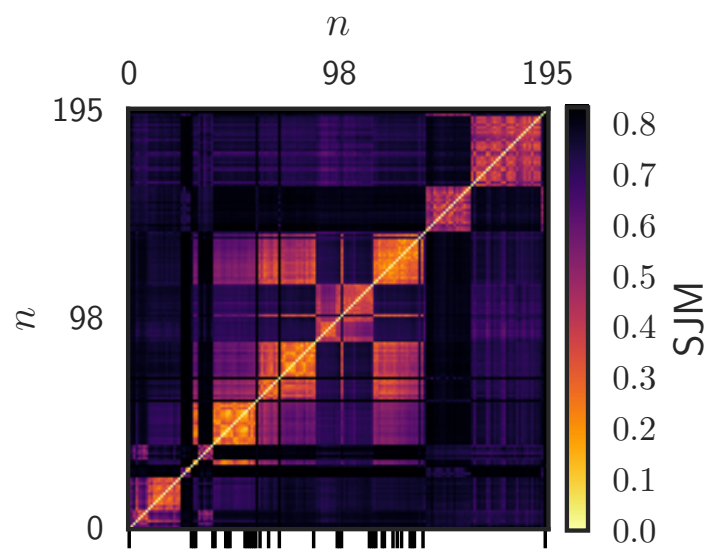

(d) SJM distance for the experiment performed on 21-06-2016

Figure 9.3.: Again the same patterns along the diagonal are made visible by both distance measures. Both methods discover similar patterns of proximity for the off diagonal elements. One downside of video based methods is visible in image c). The dark cross pattern between $n=100$ and $n=150$ coincides with the administration of additional fluorescent dye, which caused a temporary peak and subsequent nonlinear decay in image brightness.

\section{Comparison - Summary and Conclusion}

The procedures described in the previous two sections were carried out for all available data sets and the rank based Spearman correlation coefficient $\rho_{\text {spear }}$ (see 6.4) was computed between all distance matrices corresponding to a single experiment. The results 
can be seen in table 9.2. Mantel test: ${ }^{2}$ performed between matching distance matrices were highly significant ${ }^{3}$, which indicates that the observed correlations are not due to chance. Especially the relatively high correlation to the PCA based distance is interesting, as it indicates that some of the information contained in the four camera videos can be gained from a single ECG lead.

All three investigated methods to compute distances between tachycardia episodes were correlated and discovered similar distance relations between the individual episodes. In particular they all discovered the same block structures along the diagonal of the distance matrices. However as noted before the maximum correlation tended to assign lower distances to episodes that were qualitatively different in the optical signal. As optical analysis was not possible for all performed experiments this is a serious drawback of the maximum correlation, and consequently the delay reconstruction based method was used for further analysis.

\begin{tabular}{lrrr}
\hline Date & $\rho_{\text {spear }}^{\text {corr-delay }}$ & $\rho_{\text {spear }}^{P C A \text { delay }}$ & $\rho_{\text {spear }}^{\text {corr } P C A}$ \\
\hline $2016-03-09$ & 0.54 & & \\
$2016-03-10$ & 0.19 & & \\
$2016-04-14$ & 0.52 & 0.30 & 0.18 \\
$2016-06-21$ & 0.47 & 0.24 & 0.32 \\
$2016-07-08$ & 0.70 & & \\
$2016-10-26$ & 0.44 & & \\
$2016-11-25$ & 0.53 & 0.23 & 0.18 \\
\hline
\end{tabular}

Table 9.2.: Correlations between distance matrices - The Spearman correlation coefficient was computed between the different distance matrices for each experiment. Missing entries in the PCA related columns indicate that the video data could not be analyzed. Second column: Maximum correlation vs. delay reconstruction.

Third column: PCA vs. delay reconstruction.

Fourth column: Maximum Correlation vs. PCA.

Mantel tests between matching distance matrices were significant with $P=10^{-5}$.

\footnotetext{
${ }^{2} \mathrm{~A}$ permutation test with the correlation as test statistics. The number of permutations here was $10^{5}$

${ }^{3}$ The mantel tests gave $\mathrm{P}$ values which were the lowest possible $10^{5}$ permutations. In this case $P=10^{-5}$ for all tests
} 


\subsection{Analysis}

\subsubsection{Stability of the VT Morphologies under Shock}

As SJM quantifies the similarity between arrhythmias it can be used to assess how prone the observed VTs were to changes in the morphology in response to a field pulse. To this end the distribution of the distance $\delta$ between VTs directly before and after shocks was computed for each experiment, episodes that were successfully terminated or turned into VF were excluded. Low median $\delta$ signifies that typically episodes bracketed by two inductions remain similar according to our previous definition of similarity, even if perturbed. Together with the overall tendency of the heart to fall into VF this is what we will use to quantify the overall stability of the VT episodes.

On a per experiment basis it can be seen that VT changed more strongly in five of the hearts $(03-09,03-10,4-14,10-26$ and 11-25), in four of these $20 \%$ or more of the shocks caused VF. The remaining two experiments had distance distributions with low median and low $75 \%$ percentile values (06-21 and 07-08) (see 9.4), indicating that in these experiments tachycardia was less perturbed by shocks.

To eliminate the influence of the shock protocol from this analysis it was repeated in the interval $0.9 \frac{\mathrm{V}}{\mathrm{cm}}-1 \frac{\mathrm{V}}{\mathrm{cm}}$ where the experiments give enough data points to compare them at the similar field levels. As can be seen in figure 9.6 even at low fields VT in rabbits tends to change more strongly due to shock, and VT turns into VF more often. 


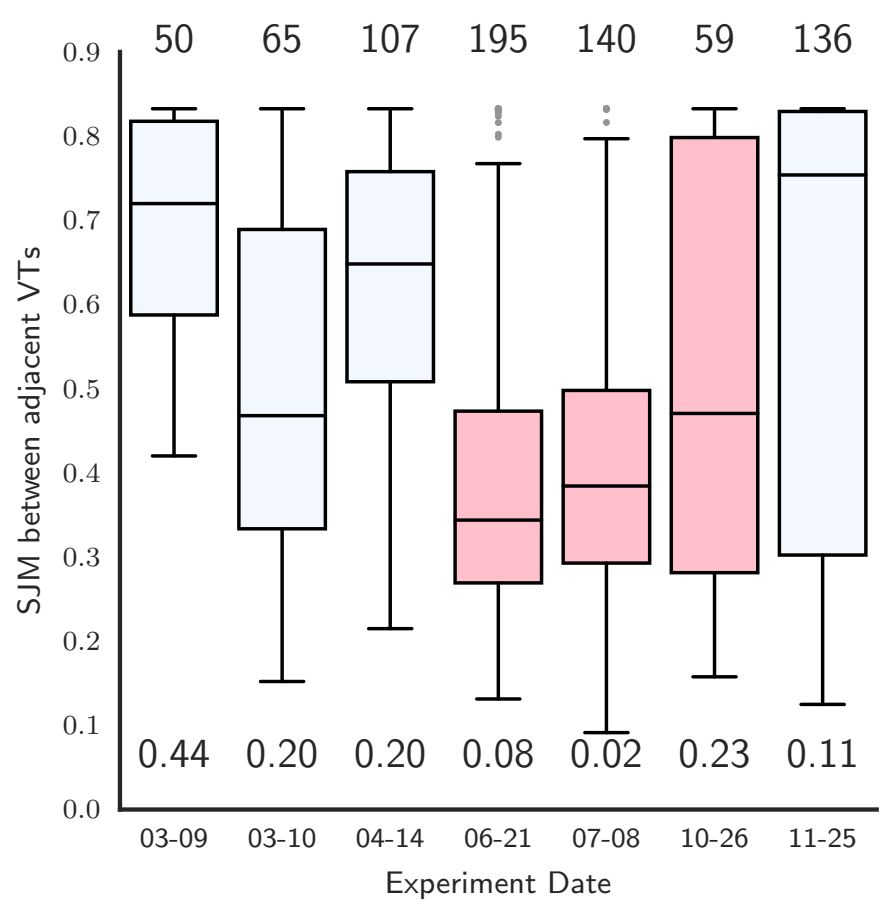

Figure 9.4.: Box plot of $\delta$ for the different experiments - The central line indicates the data median, upper and lower bound of the box are the quartiles, whiskers extend to quartile plus 1.5 time the inter quartile range. Points outside the range covered by the whiskers are drawn separately. Experiments on pigs are colored pink, experiments on rabbits light blue. $n$ is denoted above the boxes, the fraction of shocks that turned to VF is denoted below.

Median distance between adjacent episodes seems to be lower in experiments performed on pig hearts than in rabbit hearts in figure 9.4, but as different shock protocols were used on the different days (see figure 8.4 for an overview of the used fields) it is necessary to consider $\delta$ as a function of field strength and animal species to see if there is indeed a systematic difference between pigs and rabbits. Figure 9.5 shows the median SJM between adjacent VT episodes and a bootstrap based confidence interval, for the purpose of this figure all experiments have been pooled. Except for fields larger than $7 \frac{\mathrm{V}}{\mathrm{cm}}$ median change in pigs is lower than median change in rabbits. 


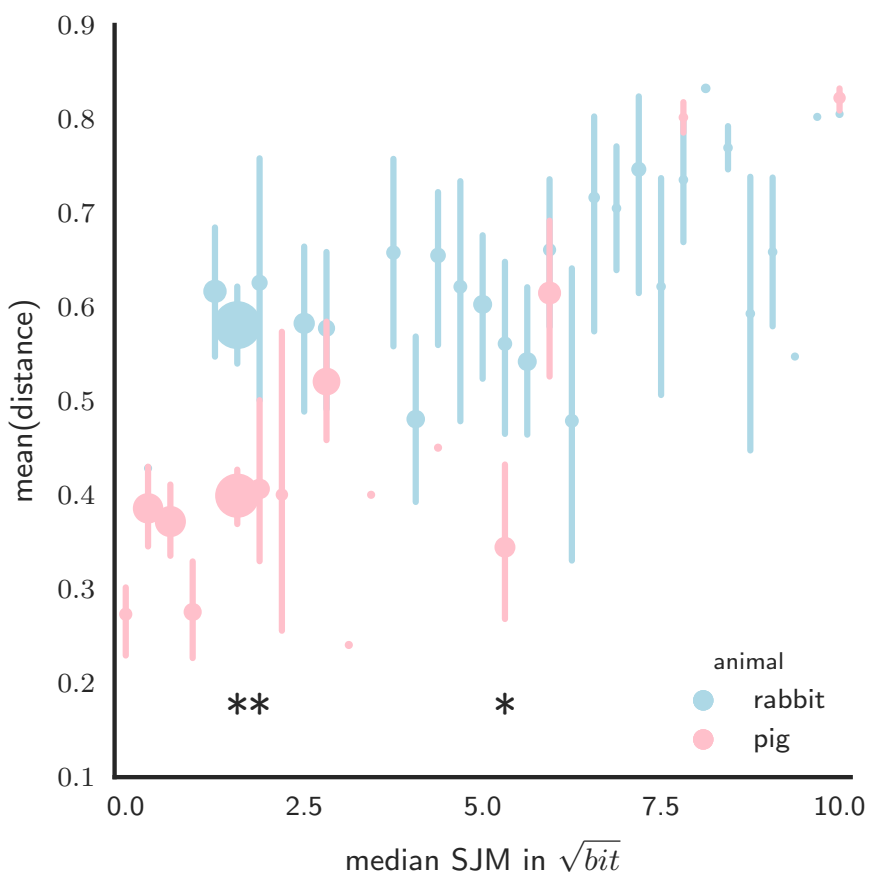

Figure 9.5.: Point plot of the median of $\delta$ for different fields and species - Median of $\delta$ as a function of field strength with bootstrap error bars. Pig data is colored pink, rabbit data light blue. Point size indicates $n$. Minimum $n$ is one, maximum $n$ 136. For shocks weaker than $7 \frac{\mathrm{V}}{\mathrm{cm}}$, median $\delta$ is uniformly lower in pigs than in rabbits. Field strength where the difference is significant are labeled with an asterix. Data of all successful experiments has been pooled for the purpose of this plot. 


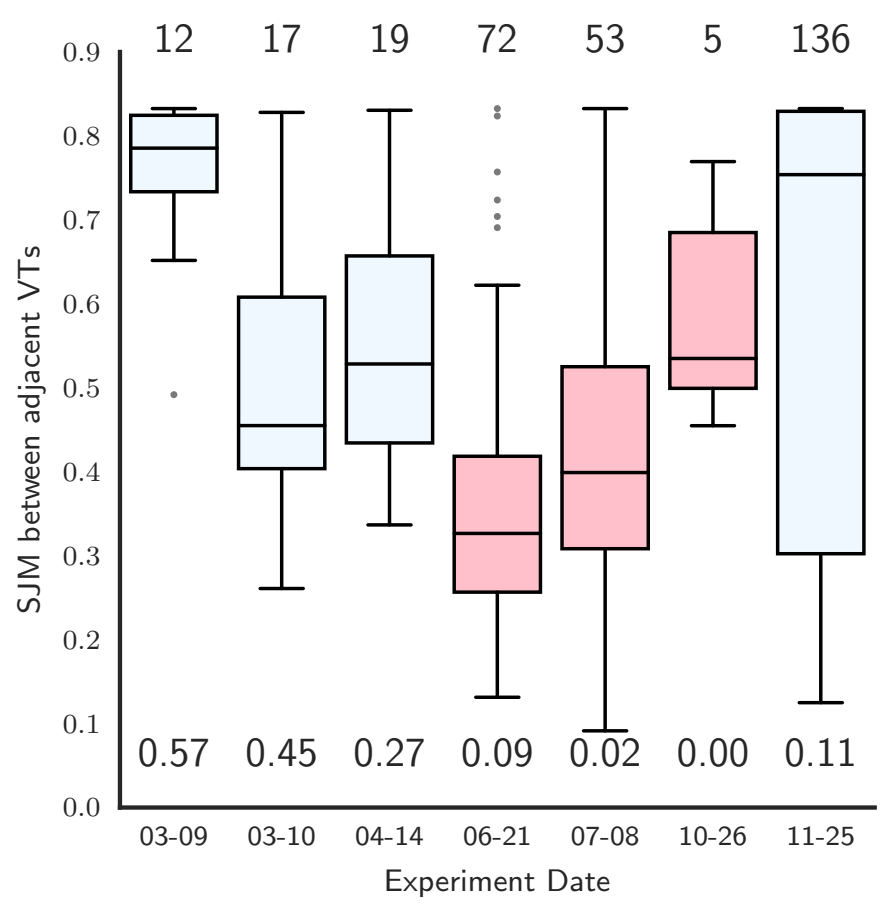

Figure 9.6.: Box plot of SJM between adjacent episodes limited to Shocks of field strengths $0.9 \frac{\mathrm{V}}{\mathrm{cm}}$ and $1 \frac{\mathrm{V}}{\mathrm{cm}}$ - Box plot details as before. To make the data comparable only shocks of similar field strength have been considered. The previously observed tendency of VT in pig hearts to be more stable is still visible.

\subsubsection{Reinduceability}

\section{Clustering}

By visual inspection of the distance matrices similarity structures were visible in all of the seven experiments (figures 9.7 and 9.8), but more pronounced in the three pig experiments (figures $9.8 \mathrm{a}, 9.8 \mathrm{~b}$ and $9.8 \mathrm{c}$ ).

In these three experiments DBSCAN found several clusters that persisted over a range of parameters $(s \in\{2, \ldots, 6\}, \varepsilon \in 0.002-0.023)$. In the case of the pig experiment performed on 2016-06-21 the clustering corresponds well to a manual grouping based on the location of the focus driving the VT. Although information about the temporal ordering of the episodes was not used, cluster membership in the pig experiments was highly correlated with time. This is in agreement with the observation made in 9.4.1 that VT on these days was relatively stable under shocks.

Cluster structures were also detected in the rabbit heart experiments, but only for larger 


\section{Experimental Results}

values of the $\varepsilon$ parameter $(\varepsilon \in\{0.045-0.38\}$, larger $\varepsilon$ lead to one single cluster containing all data). The reason for this becomes apparent when looking at low dimensional metric preserving representations of the distance matrices (see figure 9.9). While areas of higher density are visible in the pig experiments, the distribution of episodes for the rabbit experiments is more homogeneous. As $\varepsilon$ controls the length scale below which points are considered to be close, it is no surprise that for sufficiently large values some grouping is found. 


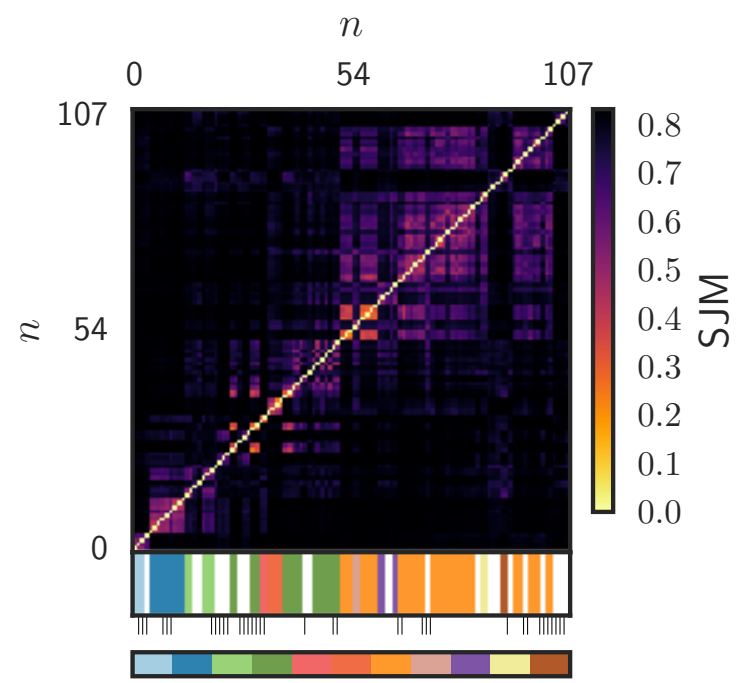

$\begin{array}{llllllllllll}0 & 1 & 2 & 3 & 4 & 5 & 6 & 7 & 8 & 9 & 10\end{array}$

Cluster Number

(a) 2016-04-14

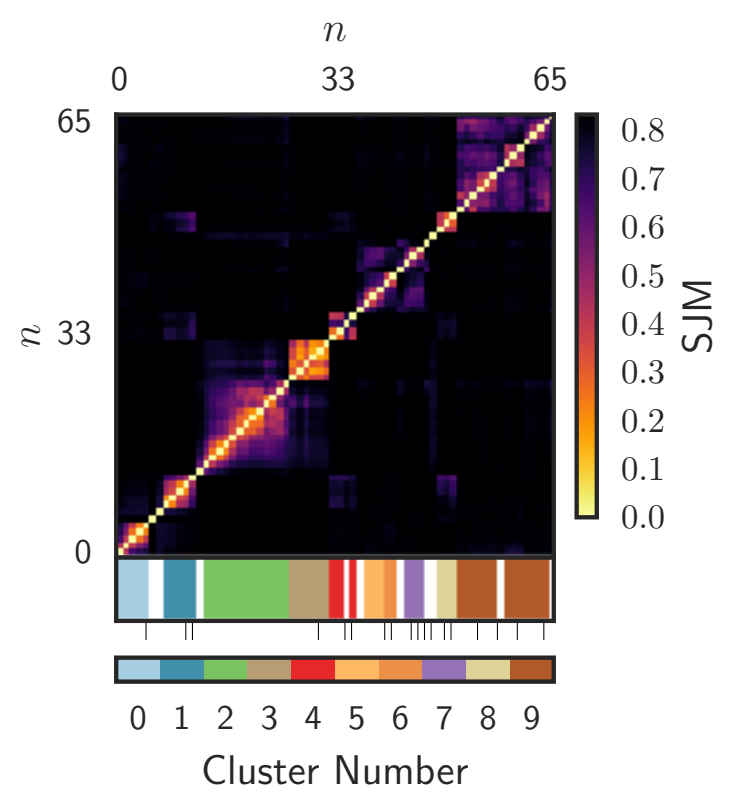

(c) $2016-03-10$

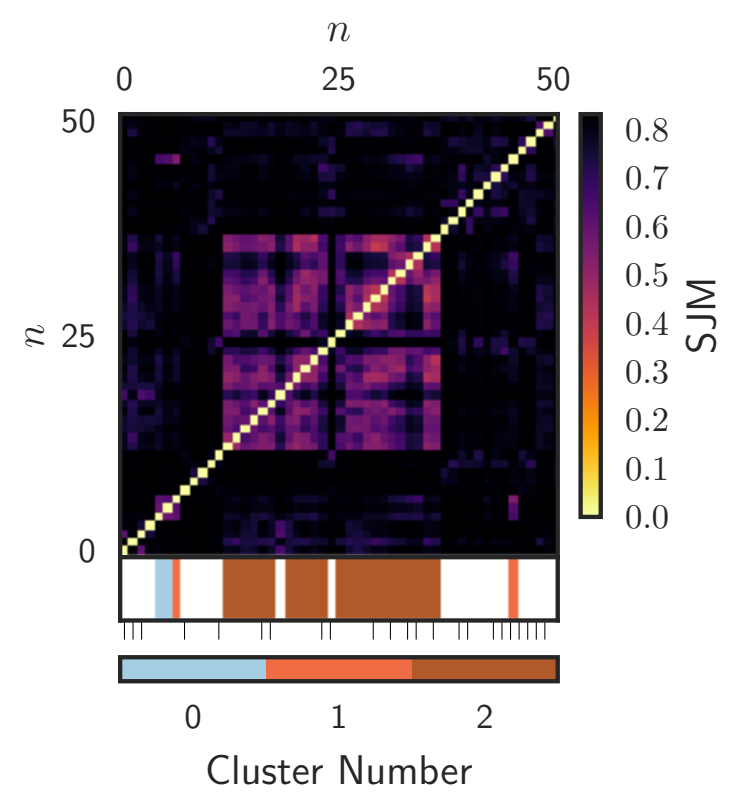

(b) 2016-03-09

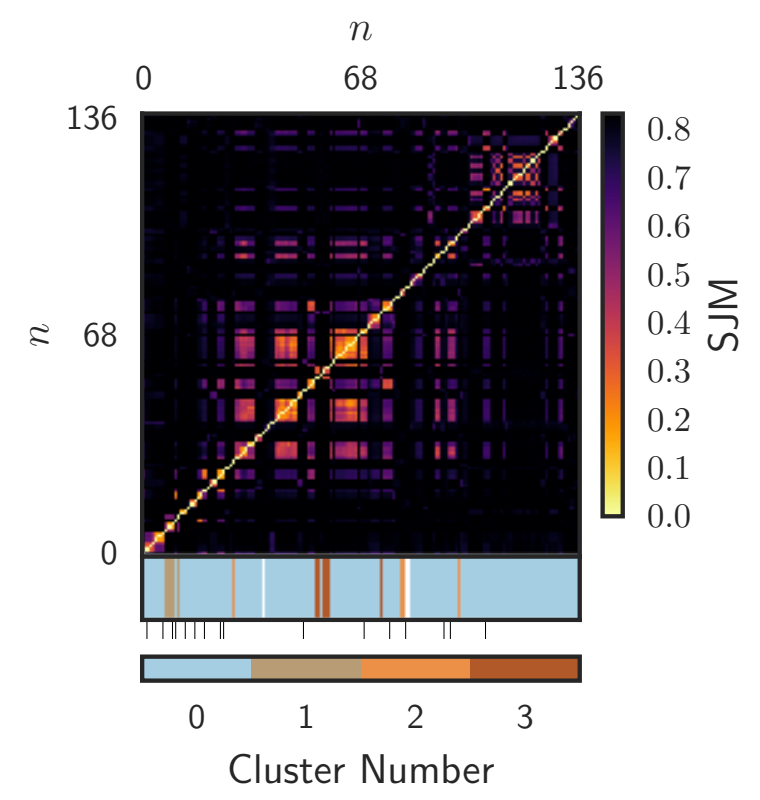

(d) 2016-11-25

Figure 9.7.: Rabbit distance matrices - DBSCAN clusters for $s=2 \varepsilon=0.2$. Cluster membership is indicated below each column of the distance matrices. White indicates that no cluster was assigned.

The shown clustering are only exemplary, as cluster membership depended sensitively on the used parameters of the clustering algorithm. Black ticks indicate new inductions. 


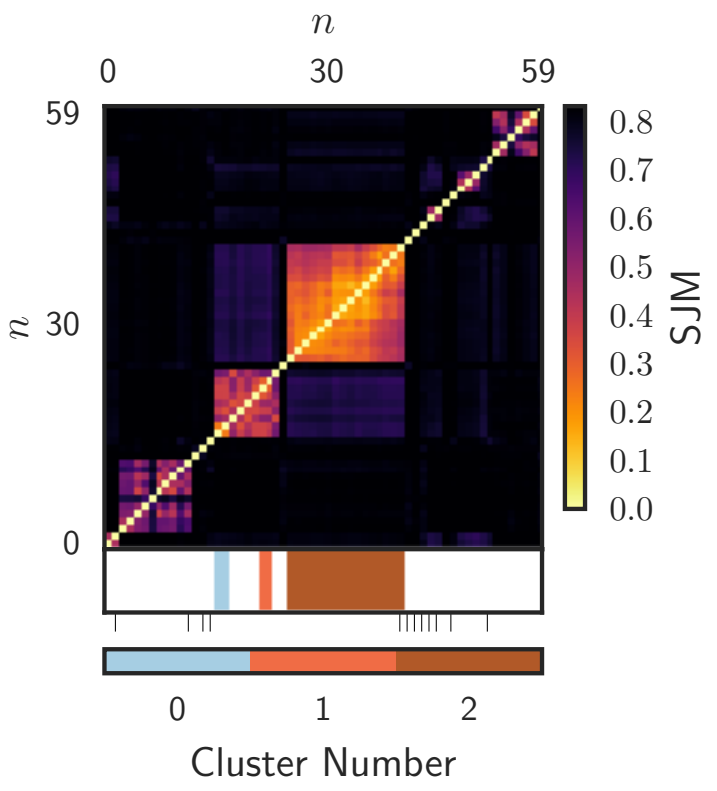

(a) 2016-10-26

2 persistent

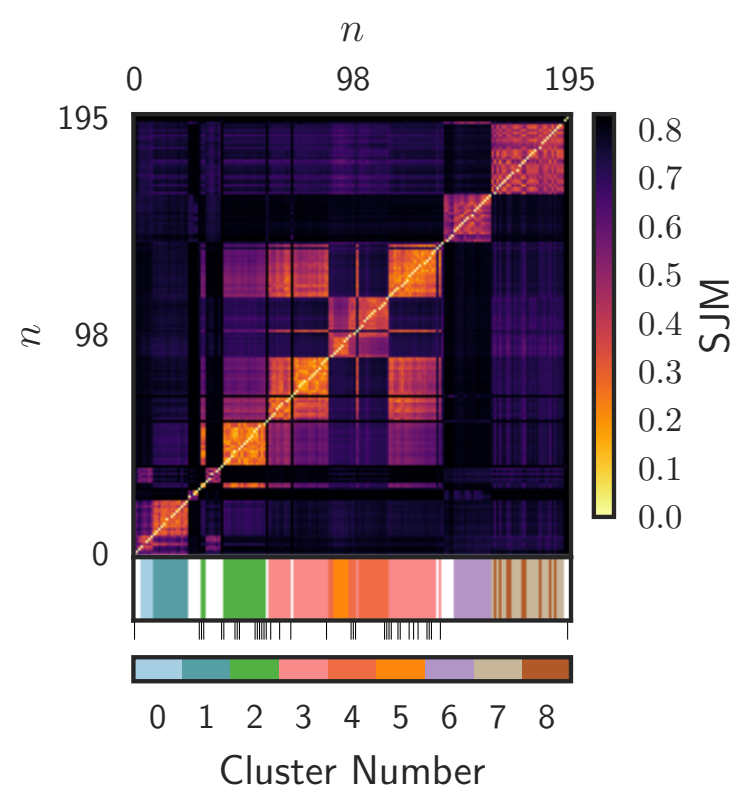

(b) 2016-06-21

2,3 persistent

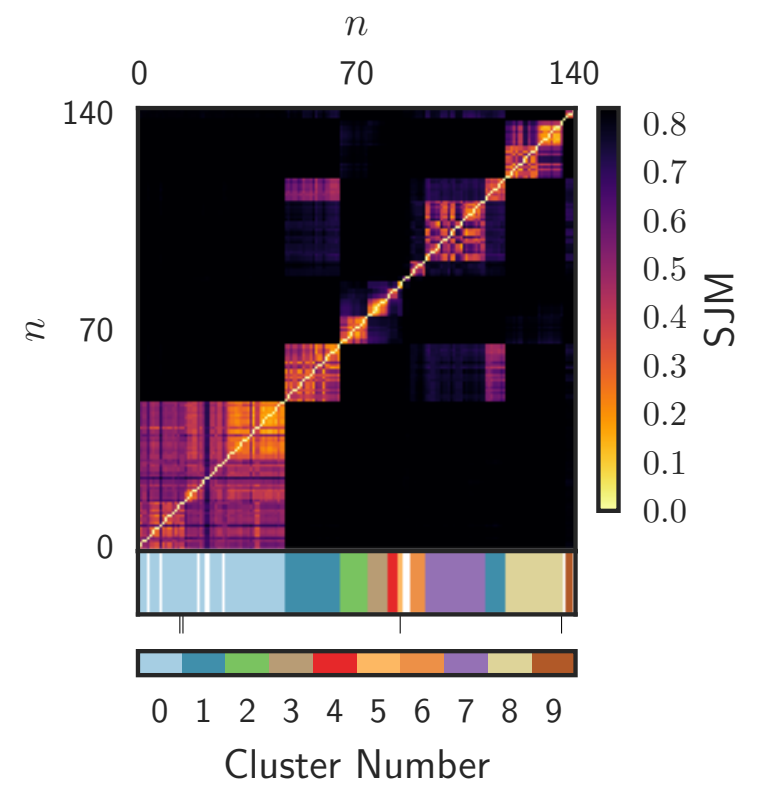

(c) $2016-07-08$
$0,1,2$ persistent

Figure 9.8.: Pig distance matrices - DBSCAN clusters for $s=2 \varepsilon=0.02$. Cluster membership is indicated below each column of the distance matrices. White indicates that no cluster was assigned.

Larger clusters persisted over a range of parameters of the algorithm. Persistent clusters are indicated in the subcaptions. 


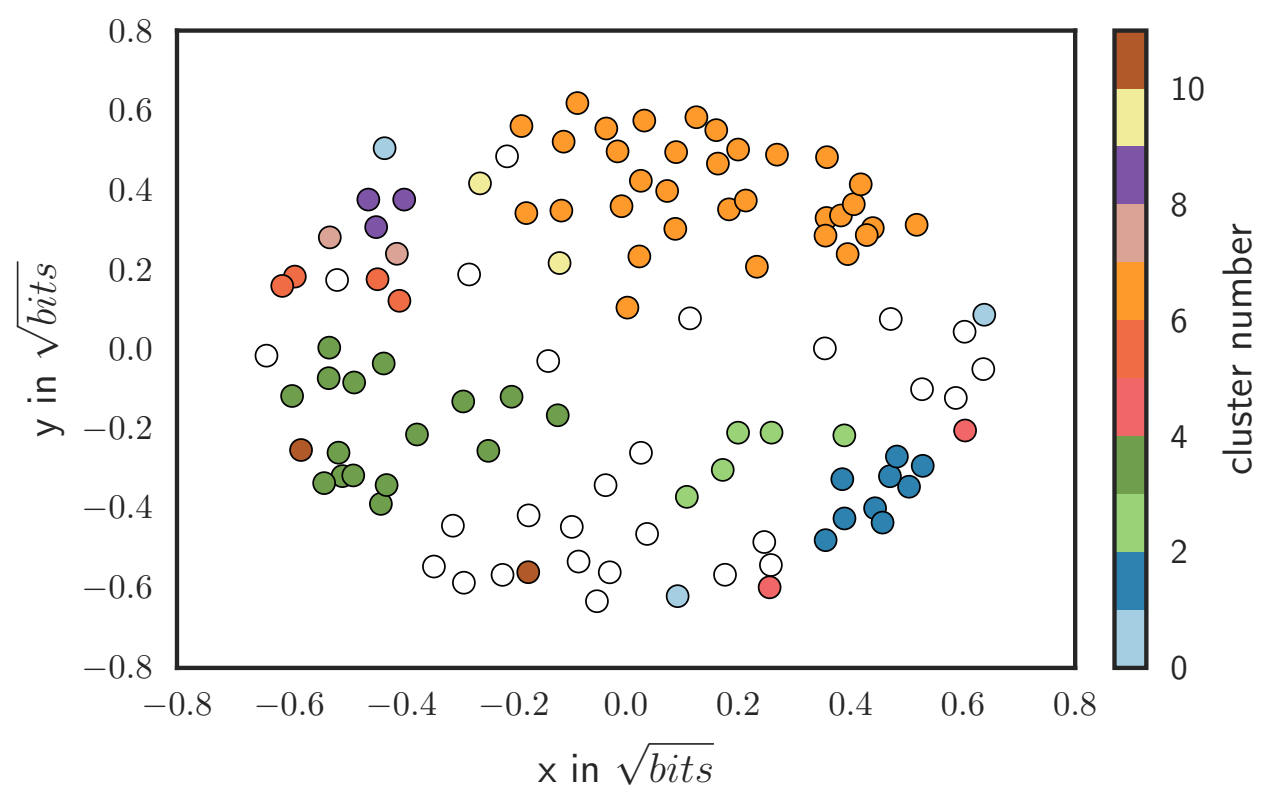

(a) rabbit 2016-04-14

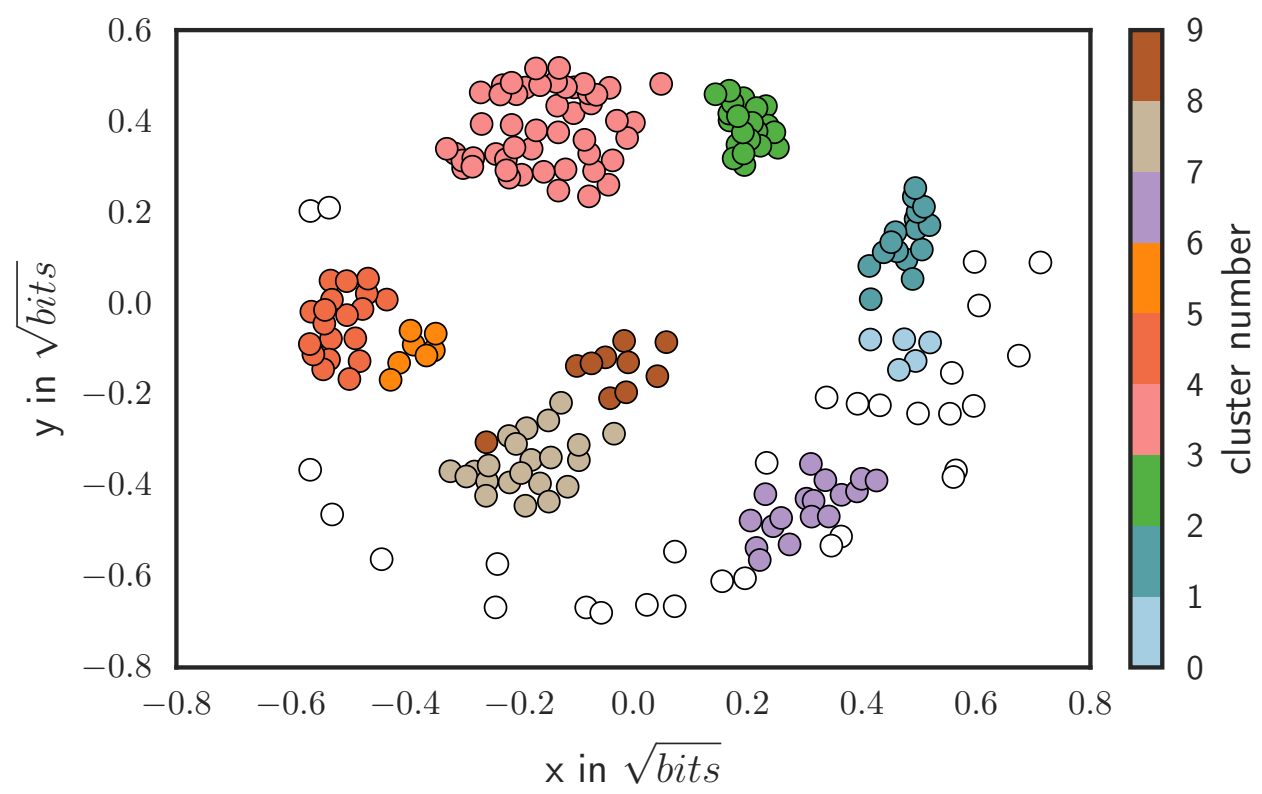

(b) pig 2016-06-21

Figure 9.9.: Low dimensional embeddings - Metric preserving low dimensional representation computed using scipys MDS function (see [59] for details on scipy and [19] for the algorithm).

Points are colored by cluster membership. White indicates that no cluster was assigned. Parameters of DBSCAN are as before.

Points in b) form clusters of high density that are visibly separated, while points in a) tend to be more equally distributed. 


\begin{tabular}{l|c|c}
\hline Experiment Date & \# Clusters & \# Reinductions \\
\hline $2016-06-21$ & 2 & 21 \\
$2016-07-08$ & 3 & 2 \\
$2016-10-26$ & 1 & 0 \\
\hline
\end{tabular}

Table 9.3.: Overview of reinductions in the pig experiments - Number of the persistent clusters, and total number of reinductions within these clusters.

Although all three experiments showed cluster structures, only in one of them tachycardia could be reinduced sufficiently often to allow further analysis.

\section{Evaluating Reinduceability}

After clustering the question of the reinduceability reduces to counting how often two separately induced VT episodes belong to the same cluster. As cluster membership depended strongly on the clustering parameters in the rabbit experiments (see 9.4.2) an answer to this question can only be given for the experiments performed on pigs.

The results can be seen in table 9.3. Of the three experiments, repeated reinduction of the same VT morphology was only possible in the experiment performed on 2016-06-21.

\subsection{Model Comparison}

Of the seven experiments that allowed for repeated induction of VT, three showed a clear cluster structure in their morphologies. In two of these experiments said morphologies were stable under shocks. Repeated induction of the same morphology was possible in only one experiment. Table 9.4 gives an overview of the seven experiments, and annotates them with the respective injury model.

\begin{tabular}{l|c|c|c|c|c}
\hline Date & animal & Injury Type & Clusters & Stable & Reinducible \\
\hline $2016-03-09$ & Rabbit & Cryo (IV) & no & no & no \\
$2016-03-10$ & Rabbit & Cryo (IV) & no & no & no \\
$2016-04-14$ & Rabbit & Cryo (II) & no & no & no \\
$2016-06-21$ & Pig & 28 days old infarct & yes & yes & yes \\
$2016-07-08$ & Pig & spontaneous & yes & yes & no \\
$2016-10-26$ & Pig & acute ischemia & yes & no & no \\
$2016-11-25$ & Rabbit & Cryo (IV) & no & no & no
\end{tabular}

Table 9.4.: Summary of the experiments

Returning to the desiderata defined in 7.2 it is now possible to compare and evaluate 
the different models.

\subsubsection{Cryoablation in Rabbits}

Cryoablation was the experimental method with the highest number of experiments that showed repeatedly sustained tachycardia $\left(N_{V T}=4\right.$ out of $N_{\text {total }}=15$ experiments). The procedure led to well defined, clearly visible injury zones on the heart surface (see figure 9.10), which acted as wave emitting sites during the whole experiment (see figure 9.11 for an representative example).

Even in the case of injuries that resulted from multiple freez-thaw cycles, optical signals taken from the injury sites showed similar overall characteristics as those take from uninjured tissue (see 9.12b); however, the fractional change in optical signal intensity as response to an action potential was greatly reduced (see figures 9.10 and see 9.12a).

A possible explanation for this observation is a thin tissue layer that contains surviving myocardial cells on top of the ablation site. As this layer is in direct contact with the warm perfusion liquid it thaws before cellular damage due to intracellular ice formation becomes irreversible.

VT could only be induced when antiarrhythmic agents were administered (Flecainide in varying doses see 8.9) and within each experiment the resulting tachycardias proved to be of low similarity (see 9.3), changed morphology frequently and often turned into VF (see 9.4.1). Terminating VT without turning it into VF was possible but only at high field strengths.

There are possibly two reasons for the low similarity of VTs in this experiments: Firstly, as the shock protocol in three of the four successful rabbit experiments (2016-03-09,201603-10 and 2016-04-14) prescribed shocks of increasing strength given at the same phase of a VT morphology until termination (see 8.9), the perturbations of close episodes were increasing in strength. Secondly, the reentrant patterns that drive the VT in this model system are not stationary: Observed spirals appeared to meander freely over the heart muscle (see figure 9.13 for an example) and changed frequently due to shock (see again 9.4.1), resulting in a plethora of different VT morphologies. Of the several hundred observed VT episodes in cryoablated rabbit hearts, no focal reentry was observed and only one was recognizable as a a pinned spiral in the video signal. This is in conflict with observations made in [60], but in the referenced publication wedge preparations of the ventricle with a large number of ablation sites were investigated rather than whole hearts.

\footnotetext{
${ }^{4} \mathrm{VT}$ morphology was classified during the experiment by hand, phase was estimated automatically
} 


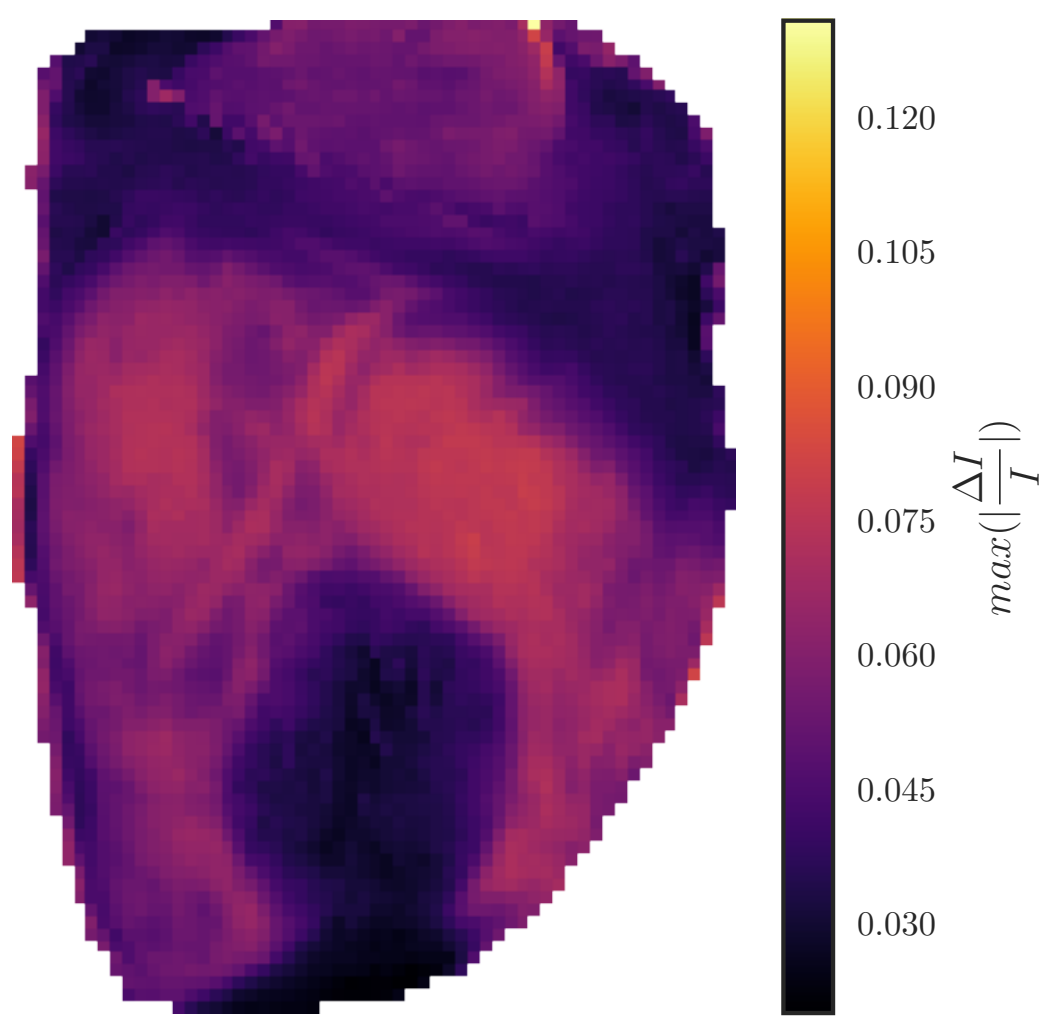

Figure 9.10.: Exemplary image of the maximum intensity change in a cryoablated rabbit heart - Anterior view of the Rabbit heart from the experiment on 2016-0309. Left ventricle is to the right, right ventricle is to the left.

The maximum intensity change as a fraction of base line intensity $\left(\max \left(\left|\frac{\Delta I}{I}\right|\right)\right.$ during normal sinus rhythm is shown at every point.

Dark areas near the top are due to fatty tissue which lacks dynamics, the well defined dark area close to the apex is the ablation zone.

As differences in staining and illumination were removed by locally normalizing with respect to baseline intensity, the reduced intensity change in the ablation zone indicates a reduced number of living cells. 


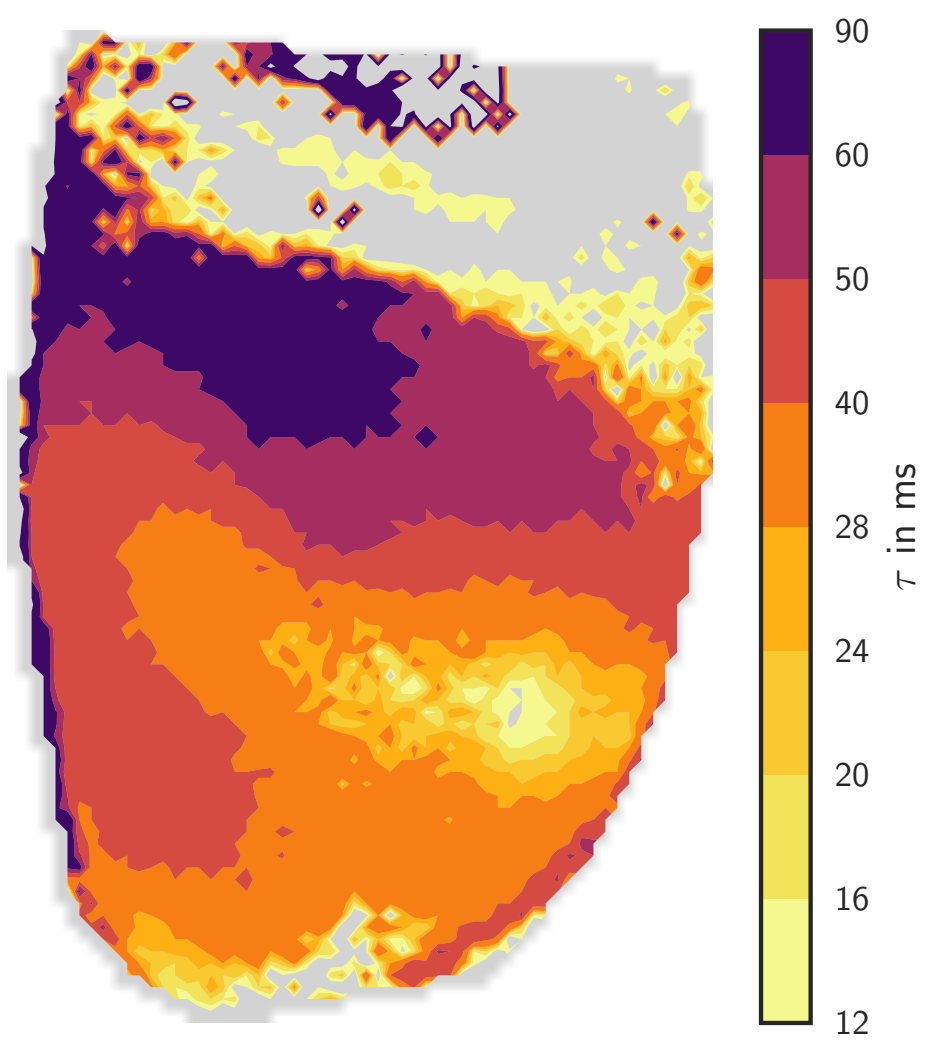

Figure 9.11.: Wave emission from the cyro zone - Rabbit heart from the experiment on 2016-03-09. Compare to figure 9.10. A $2 \mathrm{~ms}$ rectangular field pulse $0.69 \frac{\mathrm{V}}{\mathrm{cm}}$ was applied to the heart. The time until first tissue activation as a response to this pulse $(\tau)$ is shown at each point. Areas that were active at time 0 and areas that were not activated within the displayed time interval are shown gray.

As can be seen activation started at two locations: The apex and the area directly above the cryo zone and spread from there over the whole heart. 


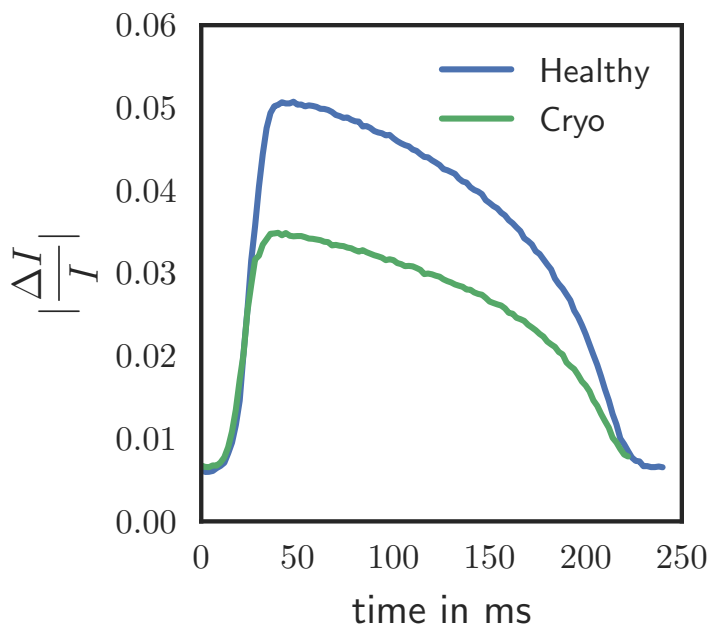

(a) Action potentials

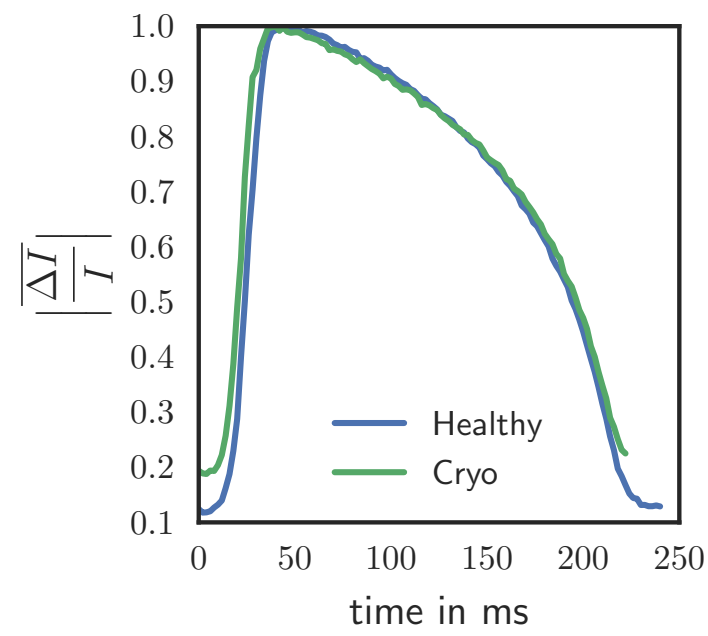

(b) Scaled action potentials

Figure 9.12.: Optically recorded action potentials - Data taken from the rabbit heart experiment on 2016-03-09 and averaged over 5 consecutive heart beats to reduce noise. The spatially averaged change in intensity as a function of time for both the cryo zone and the healthy tissue surrounding it is shown. a): Data scaled relative to baseline intensity $\left(\left|\frac{\Delta I}{I}\right|\right)$. As can be seen the relative change in intensity is bigger in the undamaged tissue.

$b)$ : Data scaled by their maxima to make comparison easier $\left(\overline{\left|\frac{\Delta I}{I}\right|}\right)$. Shape and duration of the two action potentials remain similar, but a slightly earlier upstroke within the cryo zone can be seen. 


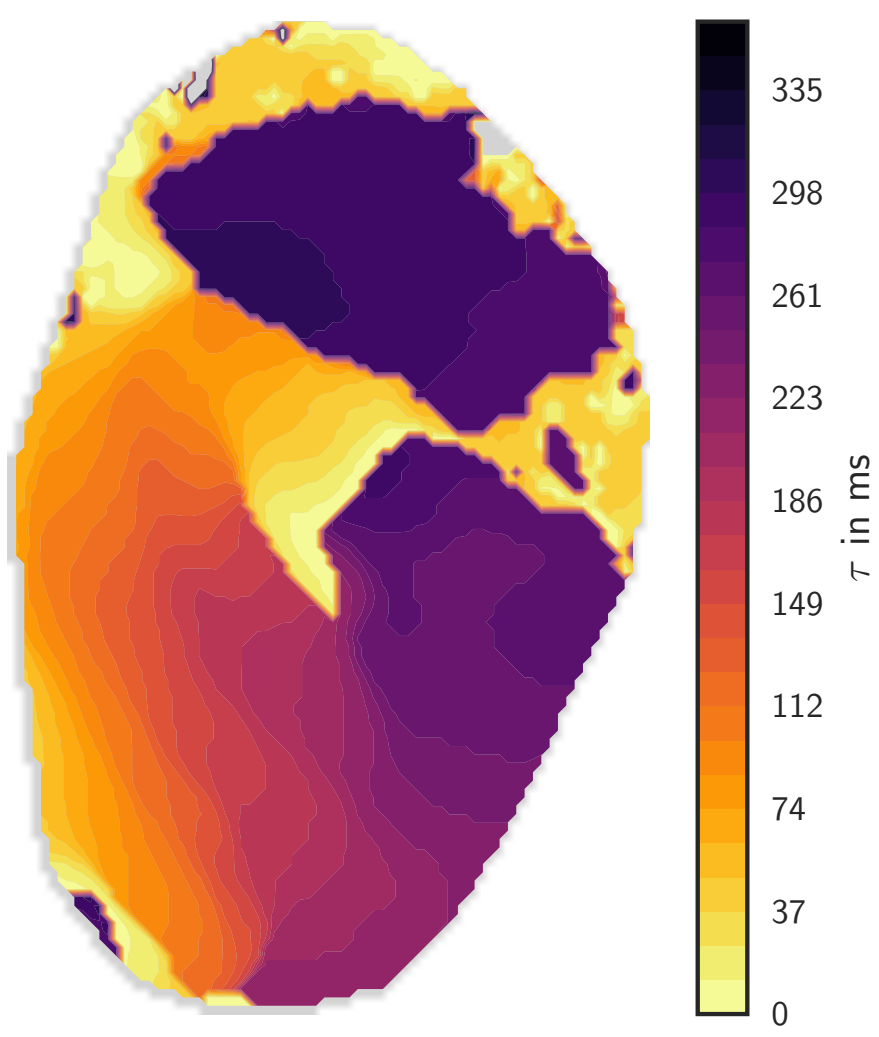

Figure 9.13.: Activation map of a typical spiral in a cryoablated rabbit heart - A typical VT episode from the experiment performed on 2016-04-14 is shown. Posterior view, left ventricle is to the left, right ventricle is to the right.

The time until first tissue reactivation $(\tau)$ is displayed at every point. The dark area near the top is part of the atrium.

The shown spiral was located on the posterior upper right ventricle, opposite to the cryoablation zone on the anterior left ventricle. Its core meandered along a linear path, which is well visible as the border line between light and dark area.

\subsubsection{Ischemia}

Three types of ischemia models were investigated ${ }^{5}$ : Reperfused ischemia in rabbits, acute and reperfused ischemia in pigs. In addition, in the one experiment where spontaneous VT was observed the reason was most likely an occlusion of one of the coronaries which lead also to an acute ischemia.

\footnotetext{
${ }^{5}$ Myocardial infarction is sometimes also grouped under the term ischemic heart disease. Here only the cases are grouped under this term where the ischemic condition is directly responsible for the changes in the tissue.
} 


\section{Experimental Results}

Reperfused Ischemia Experiments where the ischemic zone was reperfused did neither in pigs $\left(N_{\text {total }}=9\right)$ nor in rabbits $\left(N_{\text {total }}=5\right)$ yield reliably sustained VTs. In both animal models one of the anterior branches of the left coronary artery was occluded, as this is a common infarct location for humans.

In rabbits the obtained injury zones had complicated shapes with unclear extend into the muscle. When palpating lumps could be felt in the ventricular walls of all rabbit hearts near the occlusion, but ischemic zones were only visible on the surface in two of the experiments. Furthermore, as vascularization differed from animal to animal (an effect that is especially pronounced in rabbits [92]) occluding the same coronary vessels at the same location resulted in differently shaped ischemic injuries in different animals. An example of a well visible ischemic zone is shown in 9.14 .

Ischemic areas in pig hearts were more consistently visible on the surface ( 5 of the 9 experiments), although the visible areas were small compared to the heart surface (see figure 9.15). Palpating again revealed lumps in the cardiac muscle, but these were located more inside the septum and less in the ventricular wall.

Where ischemic zones were visible on the heart surface it could be confirmed that they acted as wave emitting sites in reperfused hearts (see figures 9.17 and 9.16). 


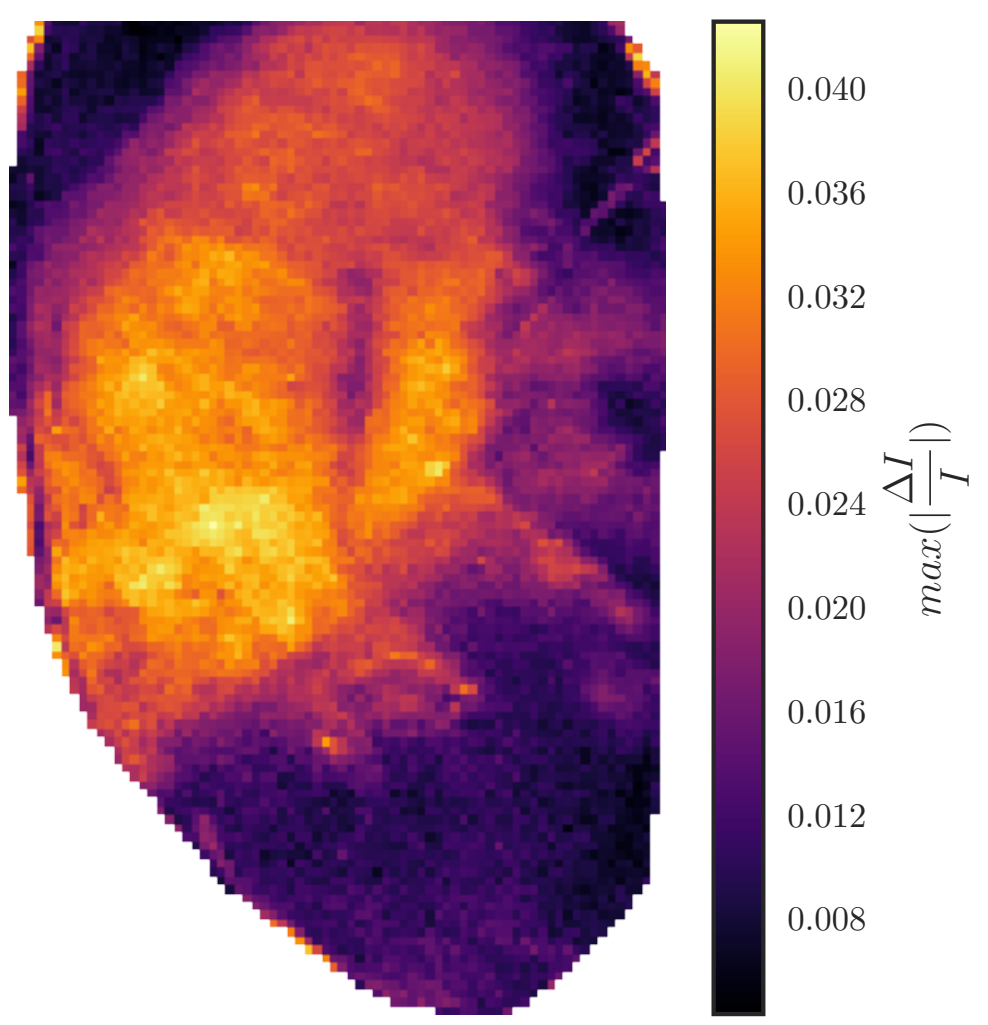

Figure 9.14.: Exemplary image of the maximum intensity change in a rabbit heart with reperfused local ischemia - Rabbit heart from the experiment on 2016-0211. Anterior view, left ventricle is to the right, right ventricle to the left. Just as in figure 9.10, the maximum fractional change in intensity $\left(\max \left(\left|\frac{\Delta I}{I}\right|\right)\right.$ during normal heart rhythm is shown at every point. The ischemic zone is well visible as dark area that extends down to the apex. Contrary to the cryoablated tissue, the ischemic tissue did not show any activation. Dark areas near the top are regions with a high amount of fatty tissue. 


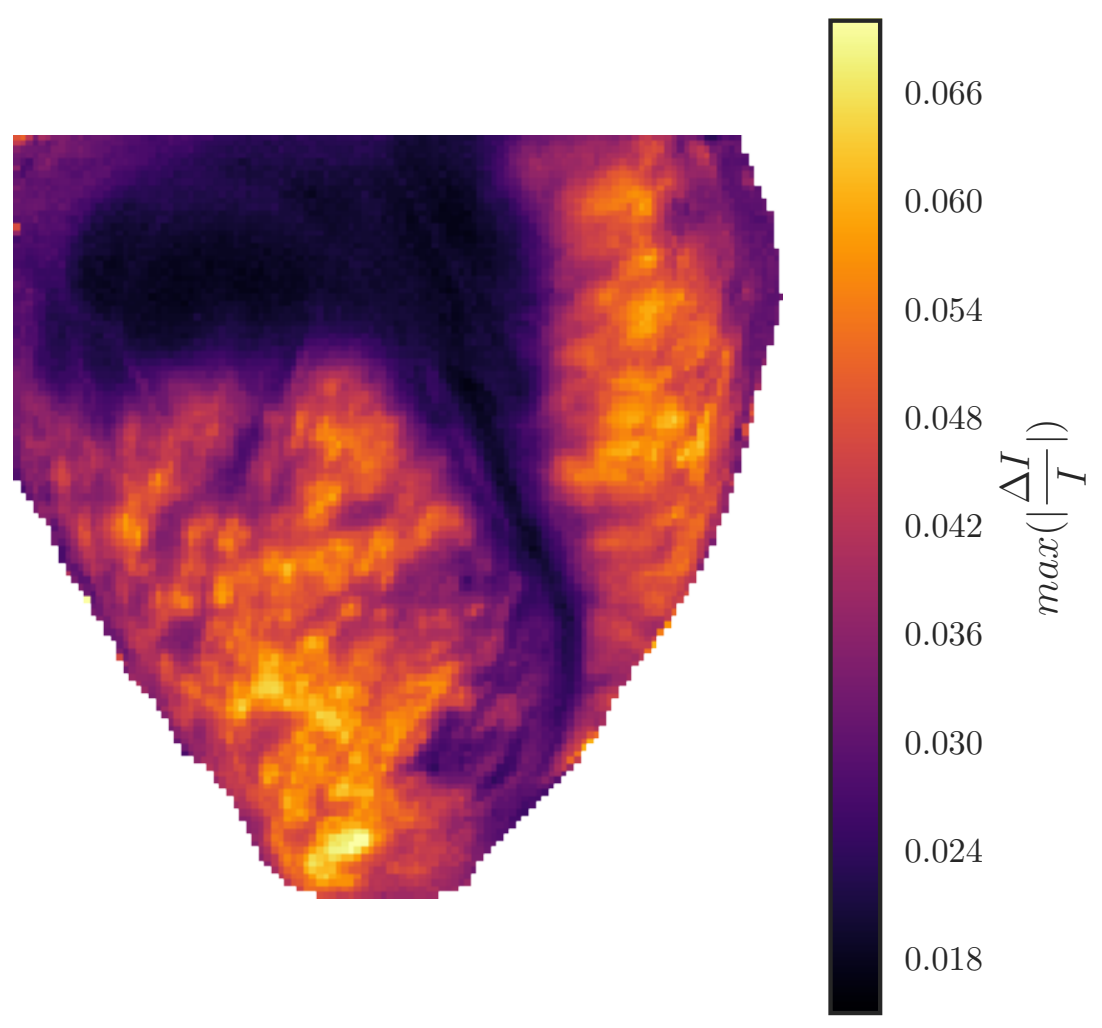

Figure 9.15.: Exemplary image of the maximum intensity change in a pig heart with reperfused local ischemia - Pig heart from the experiment on 2016-1127. Anterior view. Again the maximum fractional change in intensity $\left(\max \left(\left|\frac{\Delta I}{I}\right|\right)\right.$ during normal heart rhythm is shown at every point.

The dark area near the top contains high amounts of fatty tissue. The downward protruding dark shape to the right of the image is fatty tissue which partially covered the occluded coronary. Left to that blood vessel near the apex the ischemic zone is visible as slightly darkened area. 


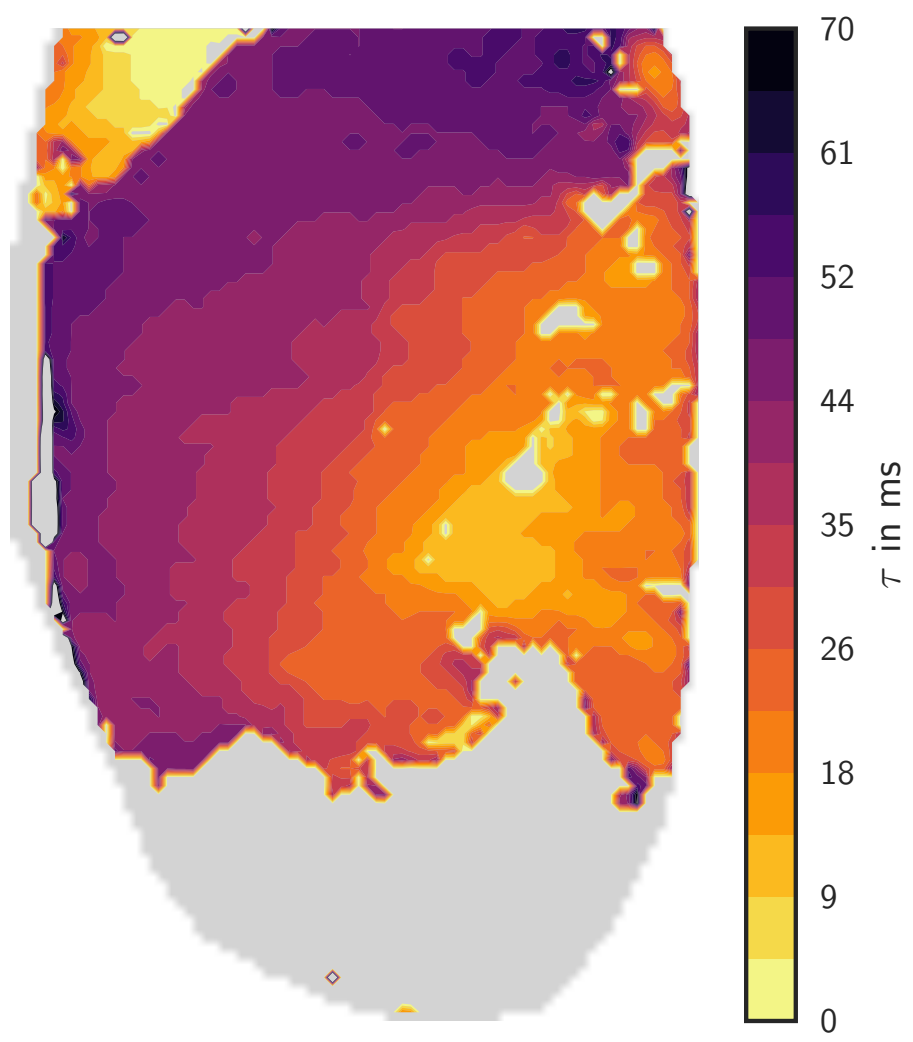

Figure 9.16.: Wave emission from the ischemic zone in a rabbit heart - Same heart as in figure 9.14. A $2 \mathrm{~ms}$ rectangular field pulse $0.51 \frac{\mathrm{V}}{\mathrm{cm}}$ was applied to the heart. The time until first tissue activation as a response to this pulse $(\tau)$ is shown at each point. As can be seen no activation occurred in the ischemic zone. 


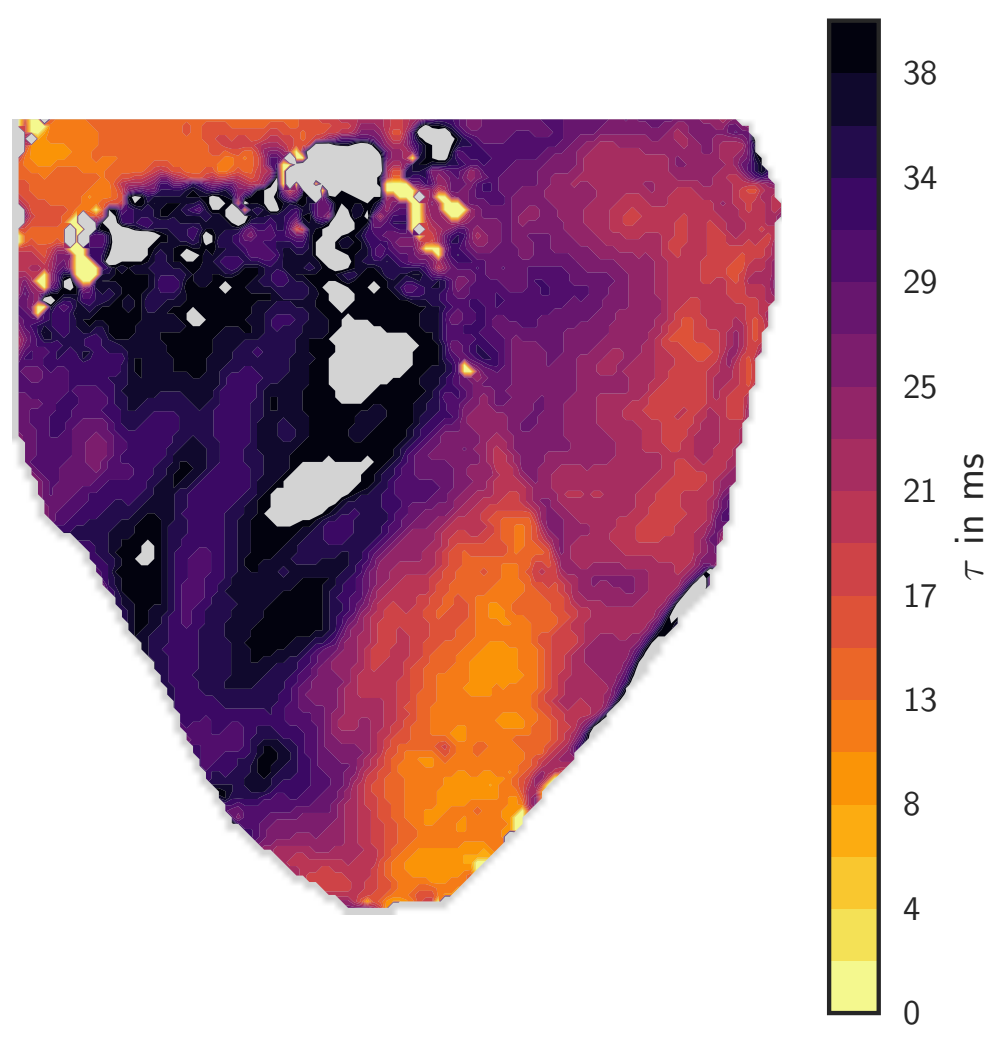

Figure 9.17.: Wave emission from the ischemic zone in a pig heart - Same heart as in figure 9.15. Image details as in 9.16. field strength $0.61 \frac{\mathrm{V}}{\mathrm{cm}}$.

Acute Ischemia No rabbit experiments with acute ischemic hearts were performed. The single pig experiment $\left(N_{V T}=1 N_{\text {total }}=1\right)$ using this model yielded several stable VT episodes, although the overall stability of the obtained VTs was low (section 9.4.1). VT was induced after the administration of the antiarrhythmic agent Ajmaline. Image quality was low due to blood in the perfusion system and movement of the heart, therefore it could not be checked whether the ischemic zone acted as wave emitting site. One persistent cluster in the morphologies could be identified, but reinduction after it turned into VF was not possible. Visual inspection of the video data showed that VT was driven by a focal reentry similar to the one observed in the chronic infarction model, but again video quality was too low due to movement artifacts to allow systematic analysis. Terminating VT without transforming it into VF first was not possible. 


\subsubsection{Myocardial Infarction}

\section{Description}

Of the six experiments conducted on chronic myocardial infarction hearts, one yielded reliably sustained VT. In this particular heart (2016-06-21) VT was stable (see section 9.4.1), reinducible (see section 9.4.2) and could repeatedly be terminated at low energies (see 9.6). As was pointed out in [80] for human patients, even healthy hearts can be forced into tachycardia, but a clinically relevant VT can be deterministicly reproduced. This experimental method is therefore the only one that provided a case of clinically relevant VT.

In all pigs the infarcted areas were either in the ventricular wall or in the septum and a direct observation of wave emission was therefore not possible. In response too weak electric pulses, excitation in these hearts started at a hotspot ${ }^{6}$ which for septal infarcts was located central on the right ventricle (see figure 9.19). This position is sufficiently close to the posterior interventricular sulcus, which marks the connection between septum, left and right ventricle, to be compatible with the hypothesis that the septal infarction scar is responsible for this. However as another possible wave emitting site, the posterior papillary muscle, is in the same region the evidence is not strong enough to proof this.

For the single experiment of this type that showed reproducible VTs (2016-06-21), the VT morphologies with cluster numbers 2 and 3 (compare figure 9.8b) were driven by focal activity located directly at the hotspot (see figures 9.19 and 9.18 ). A similar observation was made during the experiment performed on 2016-06-22, the sporadic VTs that were observed again were driven by a focus close to the hotspot (figures 9.21 and 9.22 .

\footnotetext{
${ }^{6}$ The location of this hotspot varied from heart to heart.
} 


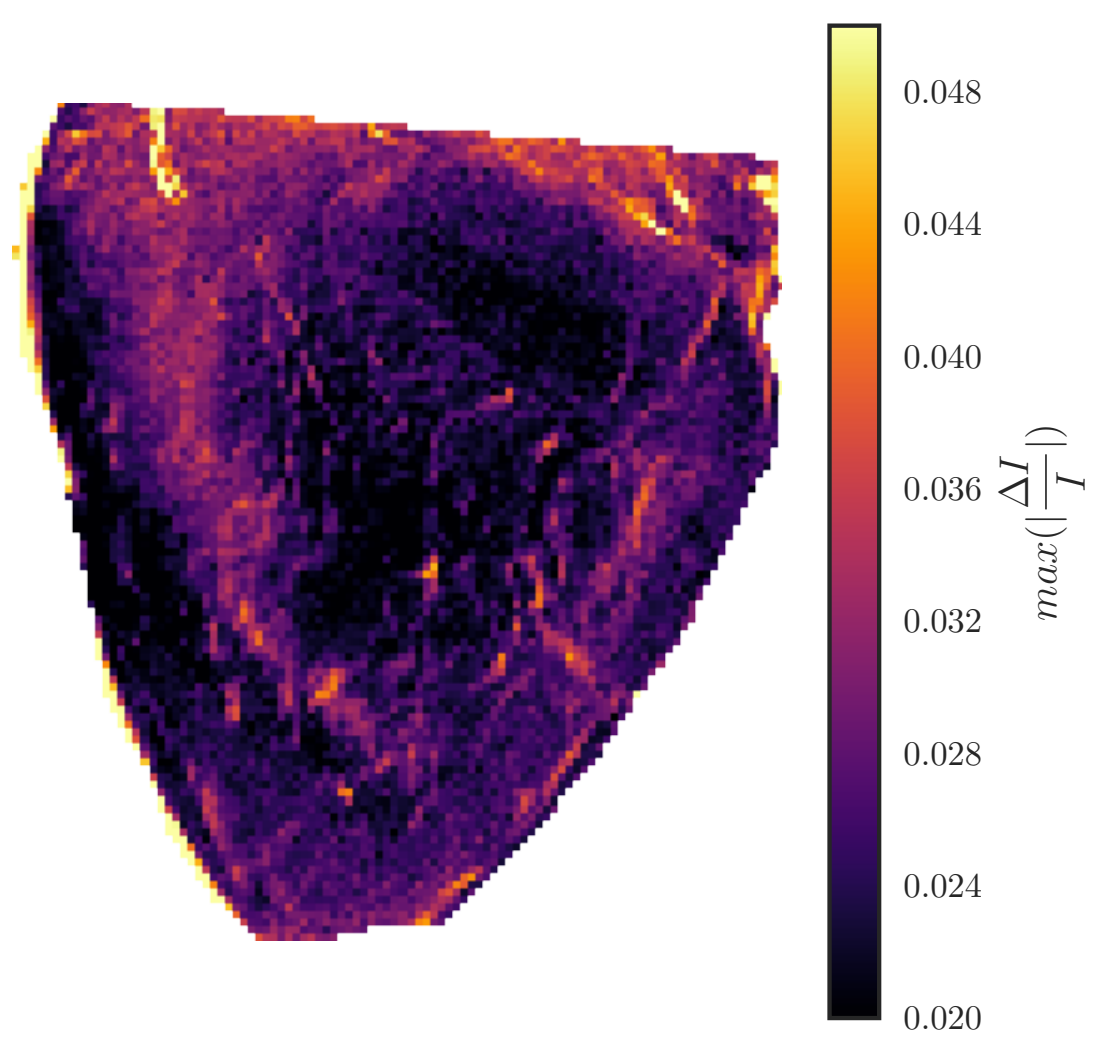

Figure 9.18.: Maximum intensity change in a pig heart with myocardial infarction - Heart from the experiment performed on 2016-06-21. Posterior view. Left ventricle is to the left, right ventricle is to the right.

Image details as in figure 9.14. The area that acted as hotspot during activation maps and VT behaved normal during sinus rhythm. Relative change in intensity did not indicate a lower amount of viable cells. Dark areas correspond to tissue with higher amounts of fat. 


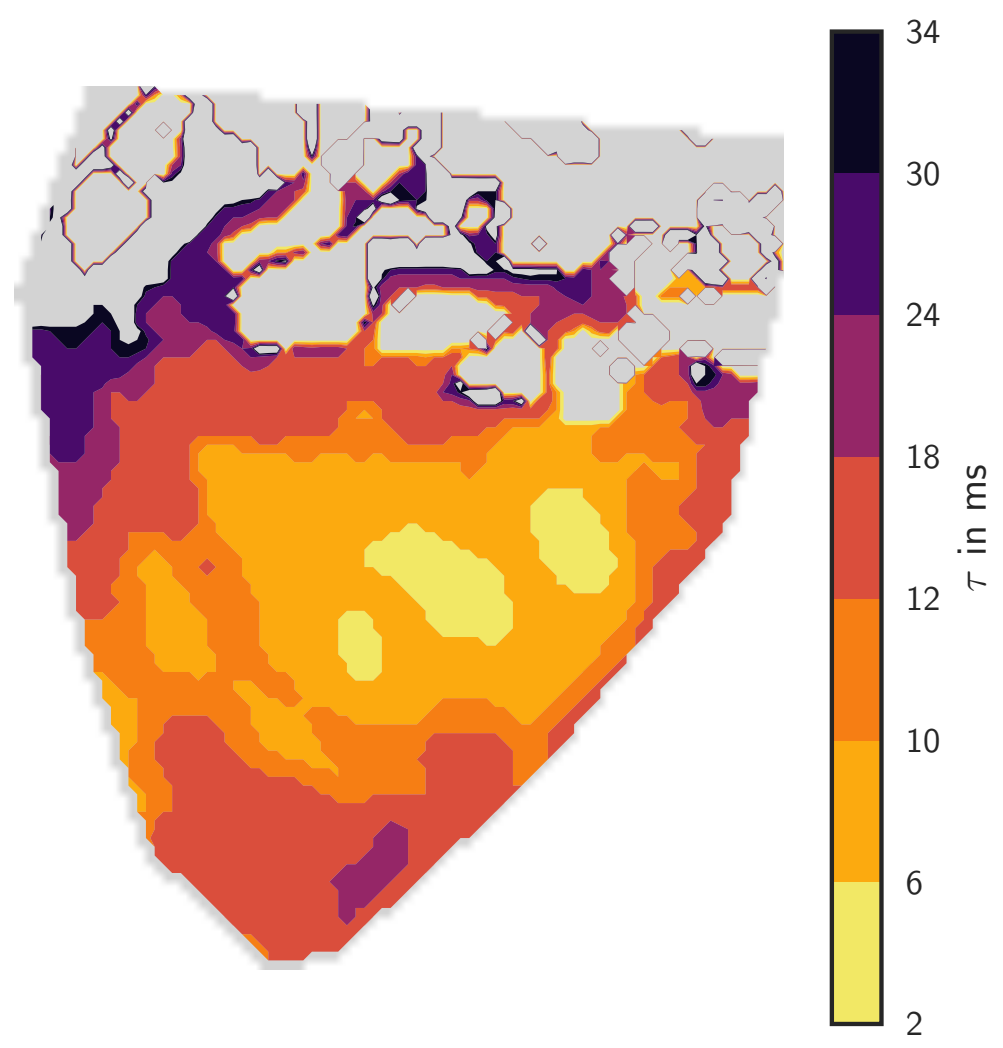

Figure 9.19.: Hot spot for wave emission in a pig heart with myocardial infarction I Same heart as in figure 9.18. Weak electric fields $0.43 \frac{\mathrm{V}}{\mathrm{cm}}$ elicited excitation at a location near the upper part of the posterior interventricular sulcus. After initiation excitation spread normally, along the fibers. 


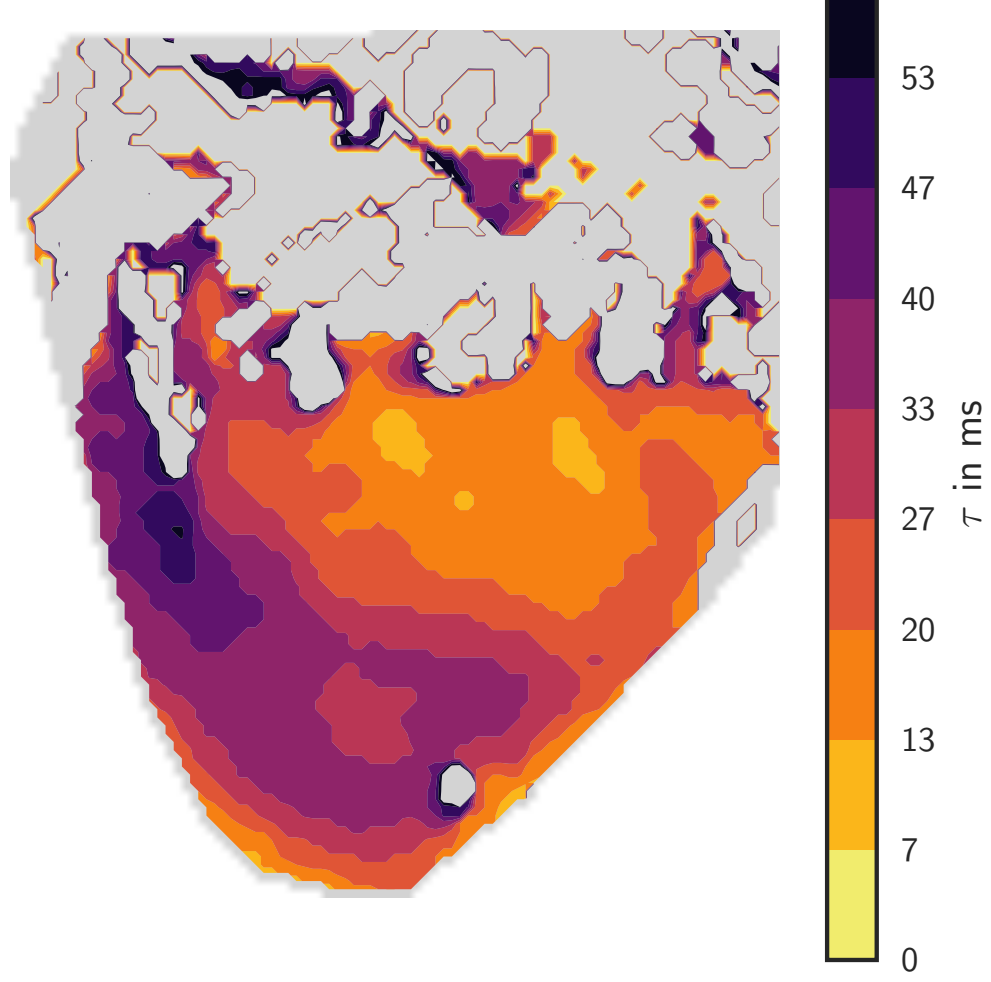

Figure 9.20.: Focal activity during VT in a pig heart with myocardial infarction - Same heart as in figure 9.18. Two of the morphologies corresponded to VTs that were driven by a focus located at the hot spot. The shown data are taken from a VT belonging to cluster number 3. Image details as in 9.11 


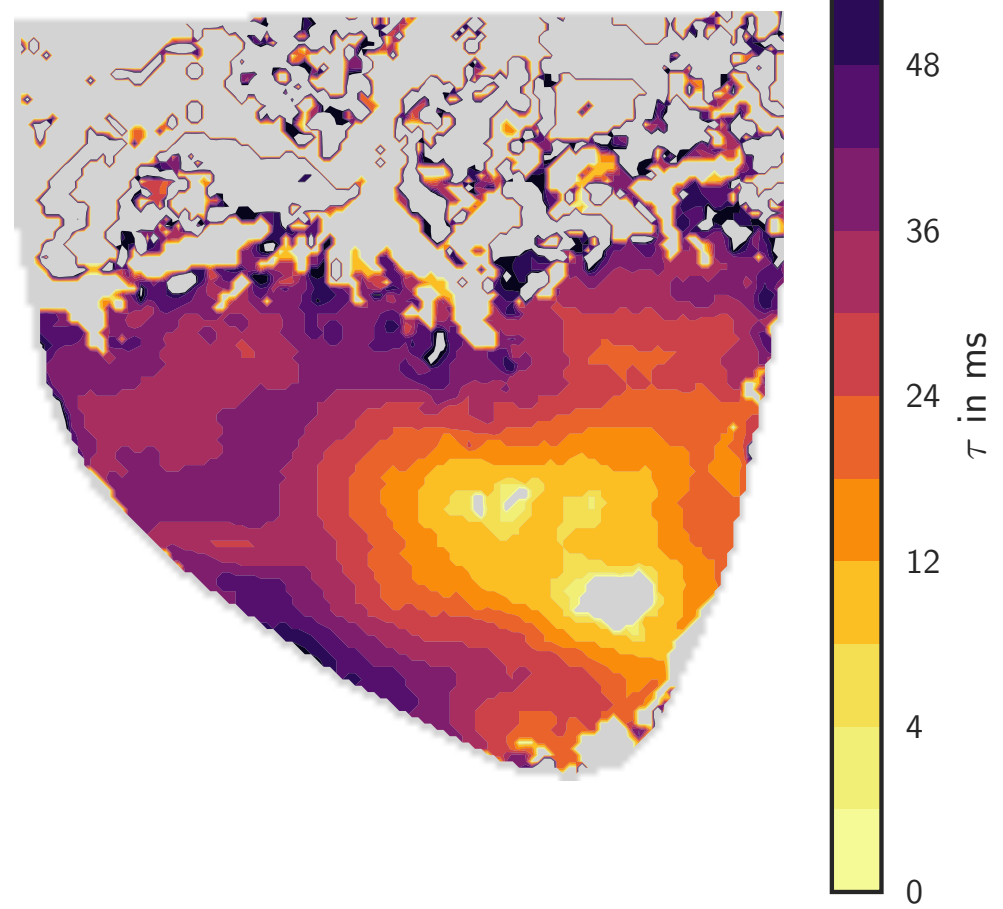

Figure 9.21.: Hot spot for wave emission in a pig heart with myocardial infarction II - Heart from the experiment performed on 2016-06-22. Posterior view. Similar to figure 9.19, activation in response to a field pulse first arose near the posterior interventricular sulcus. Field $0.17 \frac{\mathrm{V}}{\mathrm{cm}}$ 


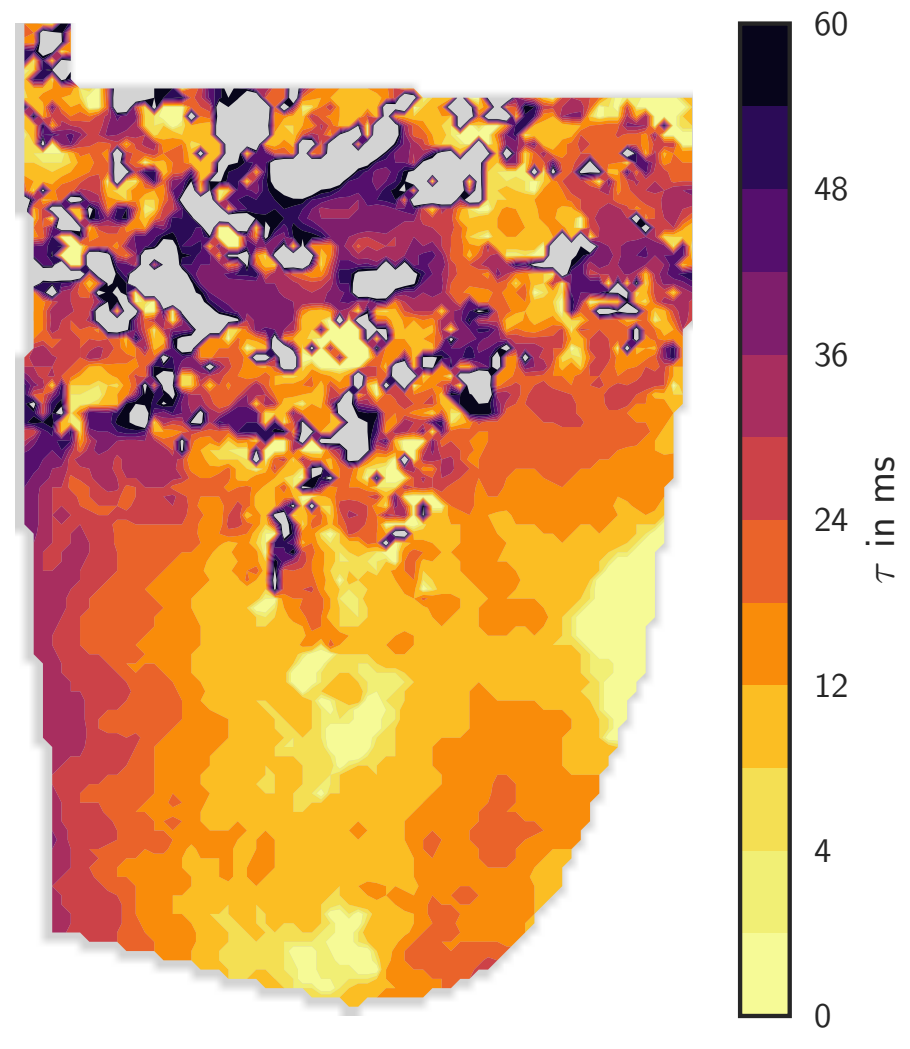

Figure 9.22.: Focal activity during VT in a pig heart with myocardial infarction II - Same heart as in figure 9.21. Still posterior view, but the image looks different as the heart moved during the experiment, and the paddle electrodes were positioned in such a way that part of the view on the ventricle was obscured. It was not possible to reliably reproduce VT in this heart, but the few short episodes that were observed, were again driven by a focus close to the hotspot. As image quality was low on this day, the show activation map was computed from data that were averaged over 3 consecutive periods of a VT.

\section{Experimental Procedure Evaluation}

In our experiments VT could be induced in myocardial infarction hearts in 1 out of 6 or differently stated in $16 \%$ of the animals. This is in crass contrast with what was reported in [129]. There VT induction was successful in $90 \%$ of the animals in a series of experiments on pigs using surgical infarct induction and VT induction protocols similar to ours. Understanding possible origins of the lower success rate in our experiments is necessary to optimize future research.

The experiment performed in [129] and ours differed in several important factors sum- 
marized in table 9.5 .

\begin{tabular}{c|c|c}
\hline & {$[129]$} & Our Experiment \\
\hline performed & in vivo & ex vivo \\
duration of occlusion & 180 min & $30-60$ min \\
premedication & Telazol & Azaperon, Ketamin \\
anesthesia & Isoflurane & Propofol, Fentanyl \\
recovery period & 6-8 weeks & $4-5$ weeks
\end{tabular}

Table 9.5.: Comparison of VT induction experiments

If one assumes that the inducibility of VT is an inherent property of the organ and therefore identical for in and ex vivo experiments, one is left with differences in premedication, anesthesia, duration of occlusion and recovery period.

Although the medication and anesthesia protocols are different, their individual effect on the development on infarcts are comparable. Both combinations are known to have cardioprotective properties that reduce infarction size, but several studies that compared Isoflurane and Propofol based infarction models found that the differences were negligible(see [63] for a review). The main difference that remains therefore is the shorter occlusion time and the shorter recovery period in our experiments. For future research it is recommended to modify these parameters to match those in [129].

\subsubsection{Conclusion}

No investigated system fulfilled all desiderata, but based on the observed behavior recommendations can be made for future studies depending on their aim.

Cryoablation in rabbit hearts with flaccid provided a way to obtain VT in approximately a third of the experiments, and all cryoablated hearts survived for a long time after attaching them to the perfusion system. Ablation zones were well visible in the optical signal and the location of the injury site could be controlled exactly. VT was driven by meandering spiral waves. For experiments that study general spiral wave dynamics on cardiac muscle, the cryoablated rabbit heart is a sufficient model, but it lacks the characteristics of a clinically relevant VT.

Although only a single experiment was performed in an acutely ischemic pig heart with Ajmaline, this model seems promising. VT was visible for extended periods of time, and seemed to be driven by a focus in a similar way to what was observed in the chronic infarction case. However, VT could not be terminated without turning it into VF first and the observed VT could not be reinduced. As this model was described 


\section{Experimental Results}

previously ([11] $)$ and the authors of that publication assure that some form of VT could be induced in all their experiments, it is prudent to assume that VT induction in this model is possible with at least the same success rate as in the cryoablated rabbit heart. This type of experiments seems therefore suitable whenever not the arrhythmia itself is under investigation, but rather imaging techniques. The acute ischemic pig heart could therefore be a useful model system for investigating inverse ECG systems ([109]), or the ultra sound imaging of cardiac arrhythmias.

Despite the small fraction of experiments that showed ventricular tachycardia, among the investigated model systems the chronic myocardial infarction model was the one that fulfilled the highest number of criteria previously defined. For research that explicitly targets ventricular tachycardia it is therefore the recommended model.

\subsection{Continued Analysis of 2016-06-21}

The special nature of the experiment performed on 2016-06-21 has been mentioned several times in this thesis: In this particular heart most of the desiderata defined in 7.2 Aim 1 were fulfilled; the observed VTs were stable, monomorphic, and the same VT morphology could be induced reproducible. Distances between the individual VTs obtained in the previously outlined way (9.3) revealed separated groups of distinct VT morphologies, applying DBSCAN to the distance matrices yielded two clusters that persisted over a wide range of parameters of the clustering algorithm (see figure 9.23). All but three successful cardioversions are within this two clusters. Although the arrhythmias were resistant to anti tachycardia pacing, VTs in this clusters could be terminated using fields comparable to the minimal field necessary to stimulate the quiescent ventricle $\left(0.7 \frac{\mathrm{V}}{\mathrm{cm}} \mathrm{vs}\right.$. $\left.0.43 \frac{\mathrm{V}}{\mathrm{cm}}\right)$. To reliably terminate ventricular fibrillation in this heart fields of $48 \frac{\mathrm{V}}{\mathrm{cm}}$ were necessary.

The mechanism of this terminations could not be determined using fluorescent imaging, but all tachycardias were special in that they were solely driven by focal activity on the heart muscle (compare figure 9.20) and the location of the driving foci was close to the main wave emitting site on the respective side of the heart (see figure 9.19). It is therefore plausible to assume that an interaction between wave emitting site and the activity at the focus is responsible for the termination success at low field strengths, although no such interaction was visible on the imaging data from the heart surface. 


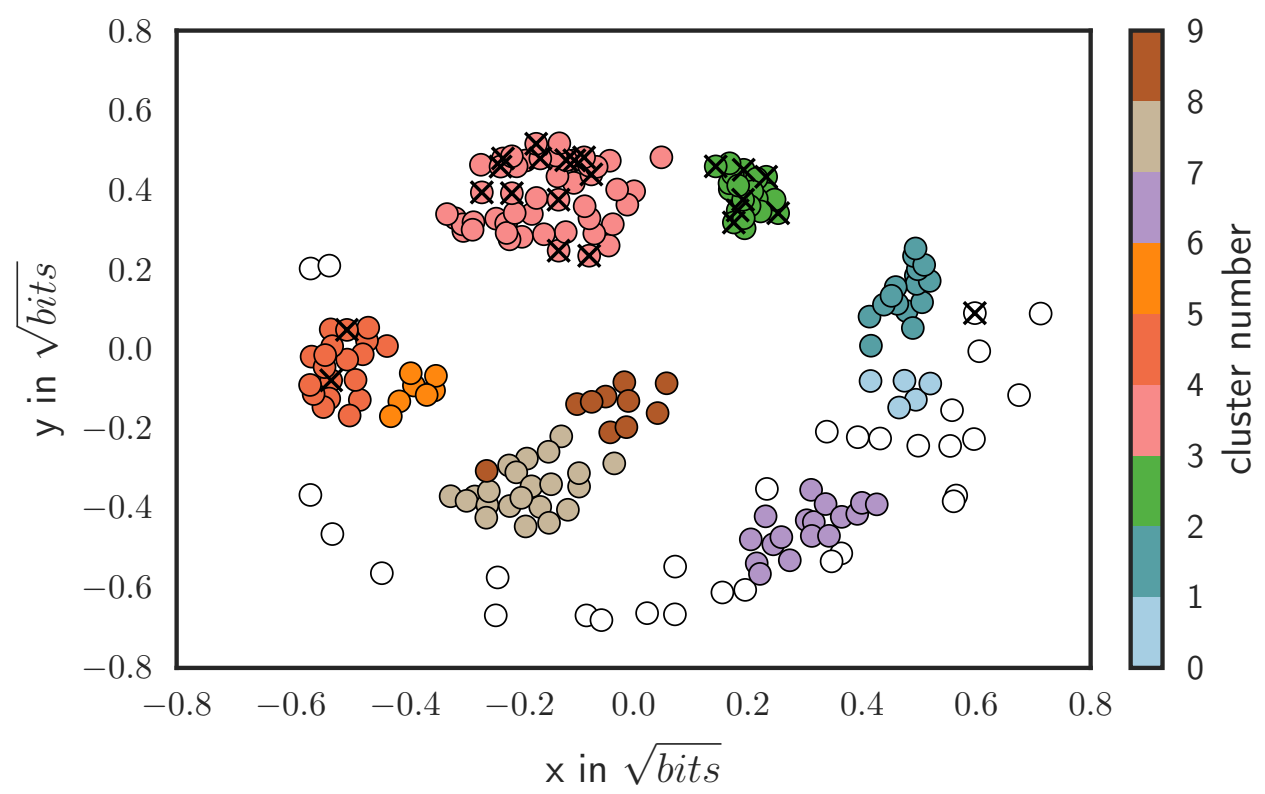

Figure 9.23.: Data from the experiment performed on 2016-06-21.

Metric preserving low dimensional representation of the distance matrix, computed using scipys MDS function ([59]).

Points are colored by cluster membership. White indicates that no cluster was assigned. Parameters of DBSCAN are as before.

All but three successful cardioversions are in clusters 2 and 3. Furthermore all low field strength cardioversions are within this clusters.

\subsubsection{Phase Dependence and Phase Transition}

The clear grouping of the tachycardias mentioned in the previous section and the fact that observed patterns on the surface were consistent for members of the cluster 2 and 3 makes it possible to investigate the phase related quantities.

To this end, for each VT a phase at time of shock onset was computed by dividing the time between the last peak in the ECG and the shock onset by an estimate of the period. Similarly, a phase shift due to shock was computed by taking the modulo of the time interval between the peaks before and after the shock with respect to the period before the shock and multiplying with $2 \pi$. Using this definitions, the phase dependence of cardioversion was analyzed (see 9.24) and phase transition curves were obtained (see figure 9.25).

In both clusters a significant phase dependence could be detected ( $p<0.01$ using the 
Kuiper test). Interestingly, despite this very clear dependence, in cluster 3 for fields of $0.7 \frac{\mathrm{V}}{\mathrm{cm}}$ and $1 \frac{\mathrm{V}}{\mathrm{cm}}$, contradicting events can be seen for higher fields.

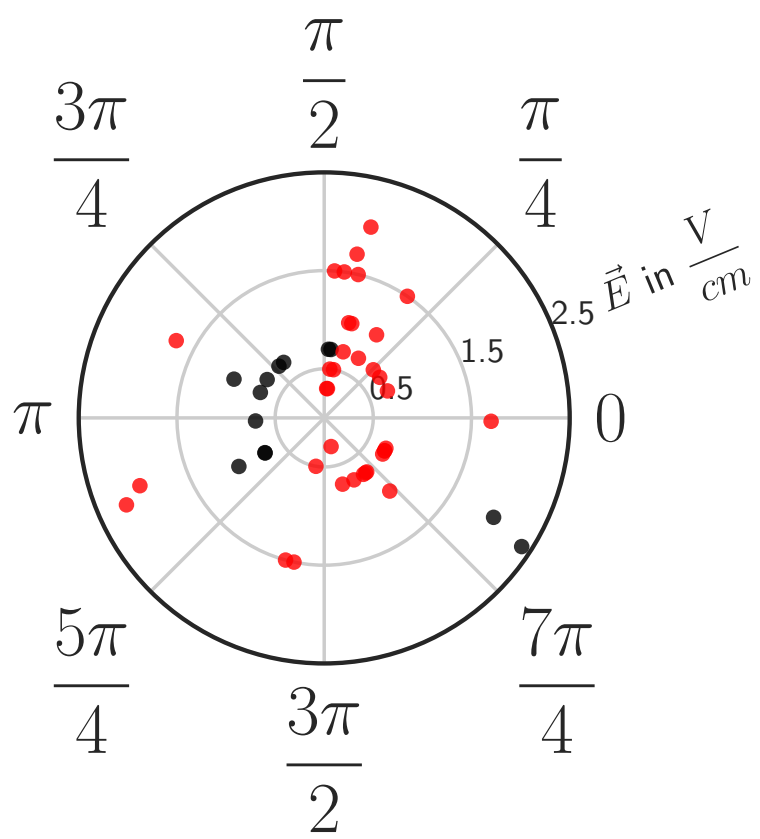

Phase in radians

(a) Cluster 3

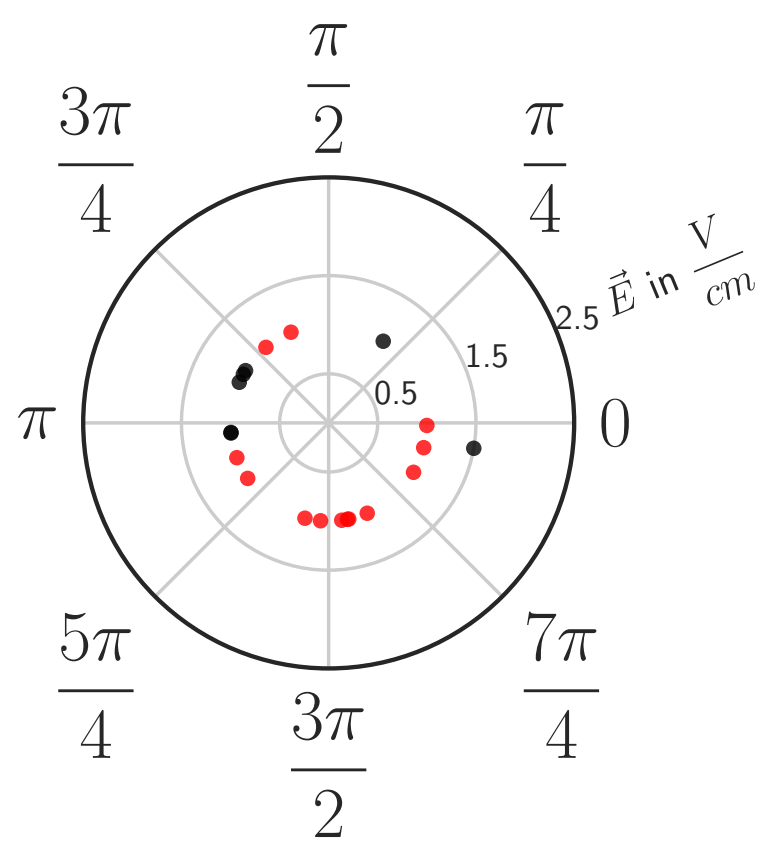

Phase in radians

(b) Cluster 2

Figure 9.24.: Distribution of termination over phase - Phase at time of the shock and field strength of the shock.

Black dots indicate success, red dots failure to terminate. Ventricular far field pacing threshold $V F F P T=0.43 \frac{\mathrm{V}}{\mathrm{cm}}$.

For VT episodes in cluster 2 and 3 termination was possible at less than twice the VFFPT, phase dependence in cardioversion success was visible. In both clusters the phase dependence was significant $(P<0.01)$ using the Kuiper test.

a) For the amplitude range $0.7-1 \frac{\mathrm{V}}{\mathrm{cm}}$ all shocks int the interval $\left[\frac{5}{4} \pi, \frac{\pi}{2}\right]$ were successful terminations. Interestingly, this did not hold for stronger fields. b) Successful shocks are located in the same region as in $a$, but not confined to that region as can be seen by the outlier to the right.

The separation in phase between successful and unsuccessful cardioversion attempts in clusters 2 and 3 is compatible with the predictions made for the unpinning of a pinned spiral (see 3.5) and the shape of the phase transition curve shown for cluster 2 conforms to what was predicted for this case (see section 3.6.1). The shape of the PTC in cluster 3 on the other hand is in conflict with the simple model presented in 3.6.1, observations made in simulations ([14]) and in cell cultures ([114]) for pinned rotors (see figure 3.15). 
Recall that the PTC introduced previously had 3 regions (I, II and III): Region I mapped phases onto themselves; region II was an interruption in the curve; region III mapped phases onto the location of the virtual cathode in phase, leading to a plateau. I and II can be seen in figure 9.27, however no part of the PTC corresponds to III. Instead an interval of sharp increase in phase (region IV) can be seen directly left of region II. 


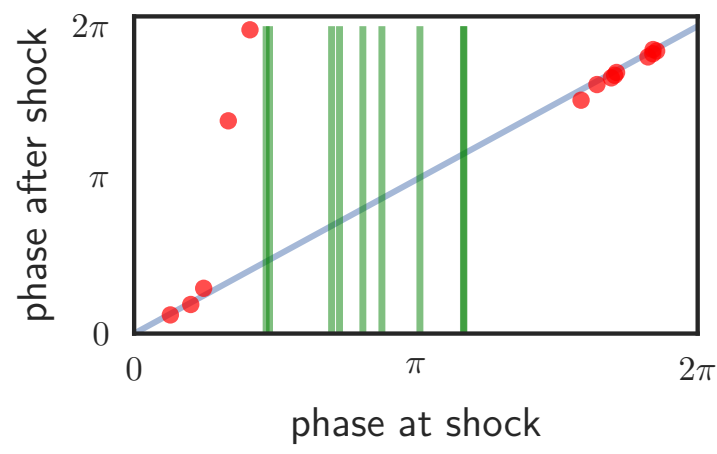

(a) Cluster 3

Data for $0.7 \frac{\mathrm{V}}{\mathrm{cm}}$ and $1 \frac{\mathrm{V}}{\mathrm{cm}}$ pooled

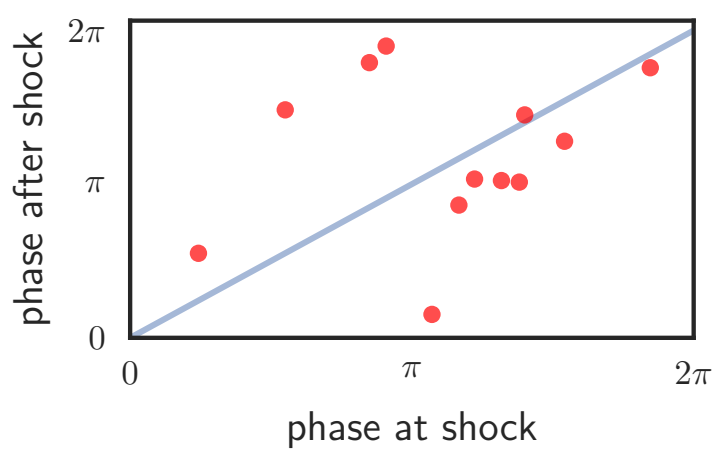

(c) Cluster 4

Data for $0.7 \frac{\mathrm{V}}{\mathrm{cm}}$

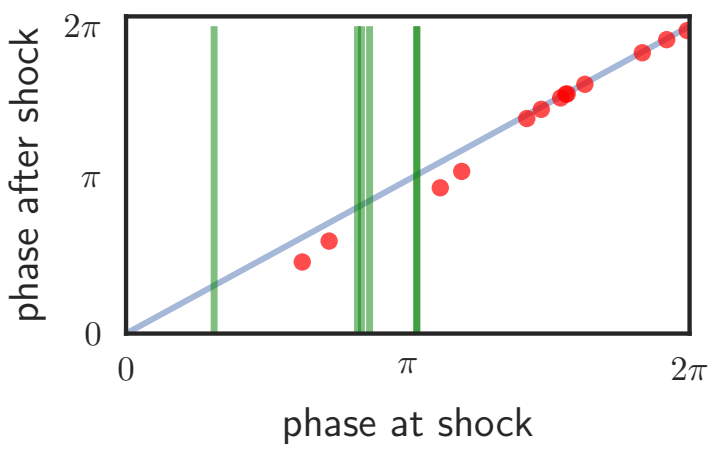

(b) Cluster 2

Data for $1 \frac{\mathrm{V}}{\mathrm{cm}}$

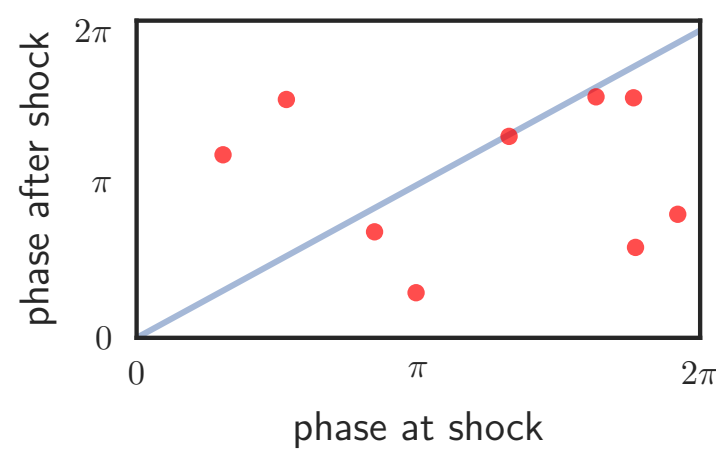

(d) Cluster 7

Data for $1 \frac{\mathrm{V}}{\mathrm{cm}}$

Figure 9.25.: Phase Transition Curves of VTI - Phase at time of the shock vs. phase one tachycardia period later for four different VT morphologies. The blue diagonal line marks the identity. Green vertical bars are positioned at phases that terminated the arrhythmia.

a) Three different regions are visible. Region I, near the right and left border. The phase before the shock and the phase after the shock are identical to within measurement precision.

Region II, in the termination window $\left[\frac{\pi}{2}, \frac{5}{4} \pi,\right]$. No phase after shock can be measured, because all shocks terminated.

Region IV: Directly left to the termination window. A sharp increase in phase can be seen.

No region corresponds to what was defined as III in section 3.6.1

b) The separation into regions is less clear than in $a$. To the right a region of vanishing phase change is visible. Around $\pi$ there appears to be a termination window similar to that of $a$; however, there are not enough data to clearly state this. Directly to the left and the right of this termination window there seems to be a region of phase decrease in response to shock. c) and d) Representative data taken at fields comparable to those of $a$ and $b$. No other VT morphology showed behavior similar to those in $a$ and $b$. No general structure in the phase transition is visible. 


\subsubsection{Discussion of the Phase Transition Curve}

The special form of the phase transition curve obtained for cluster 3 warrants a separate discussion.

Description In figure 9.26 the shocks in cluster 3 given at that $0.7 \frac{\mathrm{V}}{\mathrm{cm}}$ and $1 \frac{\mathrm{V}}{\mathrm{cm}}$ were combined into one PTC. This is done under the assumption that the phase response for these fields is sufficiently similar, since the stimuli are close to the minimal ventricular pacing threshold $\left(0.43 \frac{\mathrm{V}}{\mathrm{cm}}\right)$ and more than an order of magnitude below the required defibrillation fields $\left(\approx 48 \frac{\mathrm{V}}{\mathrm{cm}}\right)$. The phase transition behavior at the displayed field strength is consistent, the increase in phase shortly before the termination region can be seen for both field strengths. It is therefore not likely to be an artifact of the low number of data points in figure $9.25 \mathrm{a}$.

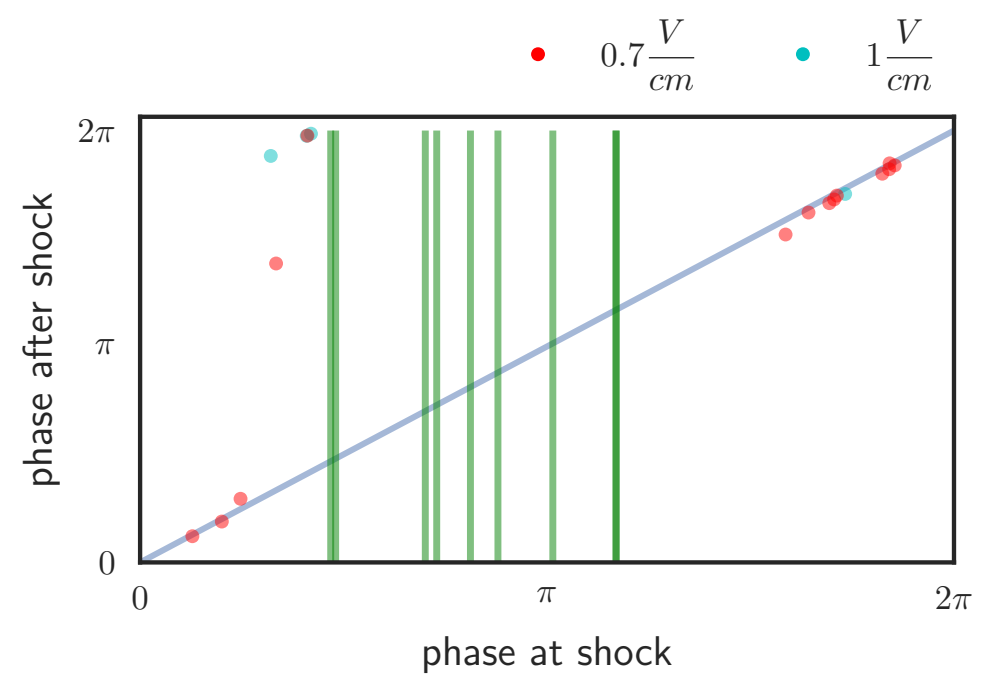

Figure 9.26.: PTCs in Cluster 3 for Different Field Strengths - The data for all fields $\leq 1 \frac{\mathrm{V}}{\mathrm{cm}}$ align very well. An increase in phase as response to a shock can be seen in all cases directly left to the termination window.

Interpretation Assuming a pinned vortex, the sharp increase in phase could be caused by either a temporary increase or a temporary decrease of the wave velocity. This is a consequence of the stroboscopic nature of the PTC. Jumping $2 \pi+\tau$ forward in phase during one period is therefore the same as being delayed $2 \pi-\tau$. Again using the model of a pinned spiral one can reason that the requirements for a wave slowing that were given in 3.6.1 are not met in region IV.

To see that recall that the ordering of the three regions of the PTC constructed in 


\section{Experimental Results}

section 3.6.1 (I, II, III) was fixed up to cyclic permutation by the ordering of the corresponding regions of the action potential (excitable window, vulnerable window, inexcitable window). A region of decreased wave propagation speed is possible due to conduction velocity restitution, but only at the border between II and III. The observed increase in phase is between II and $\mathbf{I}$ and therefore incompatible with this mechanism and incompatible with the phase transition curve as constructed in section 3.6.1.

Since the other predictions made using the pinned vortex model fit the observed data (stability under overdrive pacing, phase dependence of termination and even the existence of region $\mathbf{I}$ and $\mathbf{I I}$ ) an attempt to modify the existing models of a pinned spiral in such a way as to introduce a region IV is justifiable.

What is needed is a mechanism that temporary increases the wave speed, and indeed such a mechanism exists in form of the interaction of the action potential with local hyperpolarization ([22, p. 360]). Conduction velocity increases in hyper polarized tissue, and therefore in vicinity of the virtual anode. For small pinning centers this even could then lead to a possible collision of the wave with its own tail, which would provide an additional possible explanation for the observed unpinning window. The described situations are visualized in figure 9.28 .

In figure 9.27 an extension of the simple axiomatic model that was introduced in section 3.6.1 is presented, it incorporates this effect. This new model is again based on four considerations: Except in the regions of hyperpolarization, the spiral propagates with constant velocity around the boundary. Hyperpolarization increases the wave speed. The locations of virtual electrodes are fixed; depending on the position of the action potential with respect to the virtual cathode only three things can happen:

I The electric field is too weak for the virtual cathode to have any influence. Shocks that fall into phases where the action potential covers the cathode do not cause a shift in phase.

IV Waves that are near the end of the virtual anode at time of shock, are only accelerated by the hyperpolarization and jump forward in phase. This shift increases with the distance the wave travels in the hyperpolarized region (see figure 9.28a ff.).

II At some critical point in phase the jump forward in phase is do pronounced that the wave collides with its own tail and is unpinned $(9.28 \mathrm{~d}$ ff.).

This PTC again has three regions, which are labeled I, II and IV. I and II are phenomenologically identical to their counterparts. IV is the desired region of rapid phase increase. The possibility of the existence of region III is not excluded. If one would drop the assumption that the cathode has no influence on the curve an additional unpinning window and a plateau would arise. This would result in a mixture of the PTC displayed in figures 9.27 and 3.14 . 


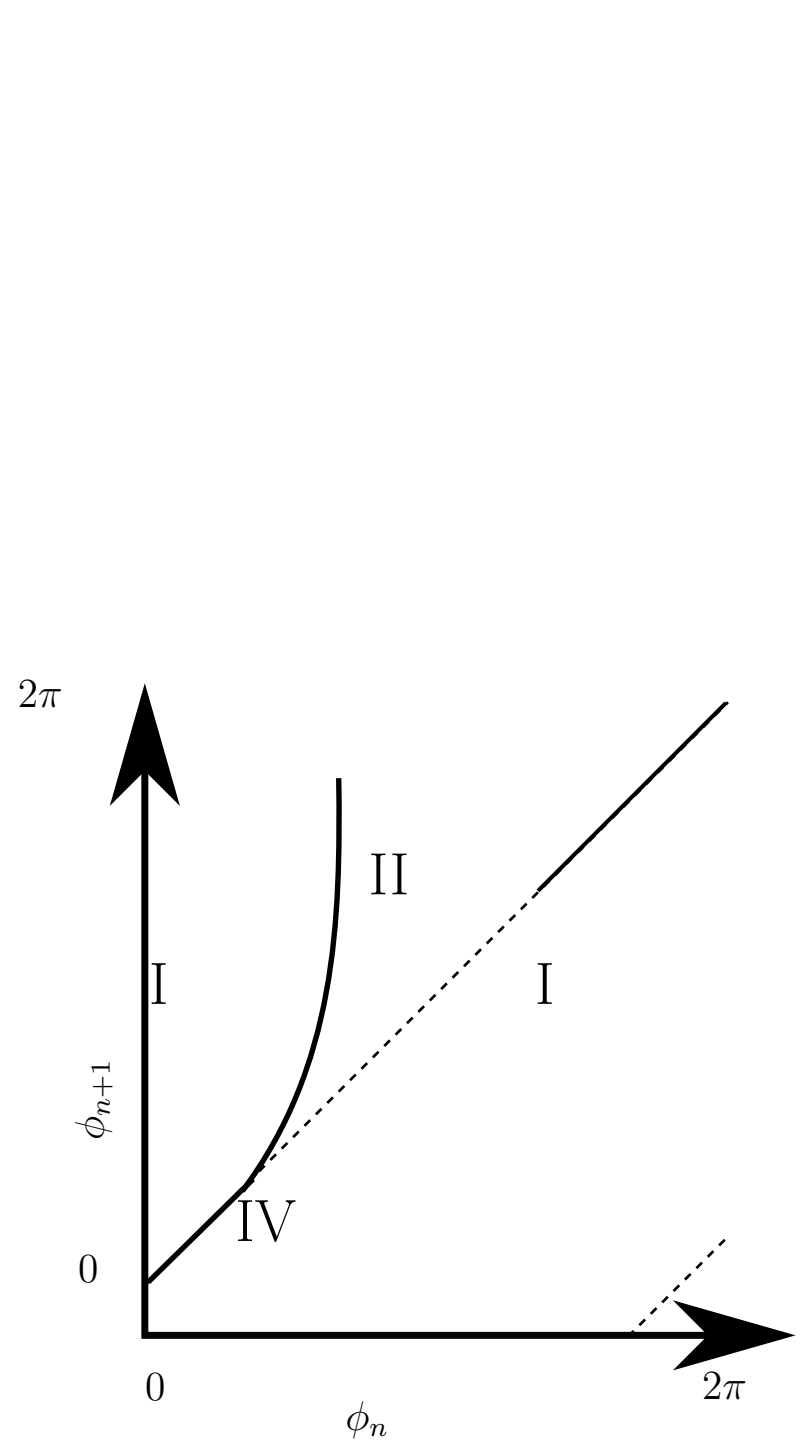

(a)
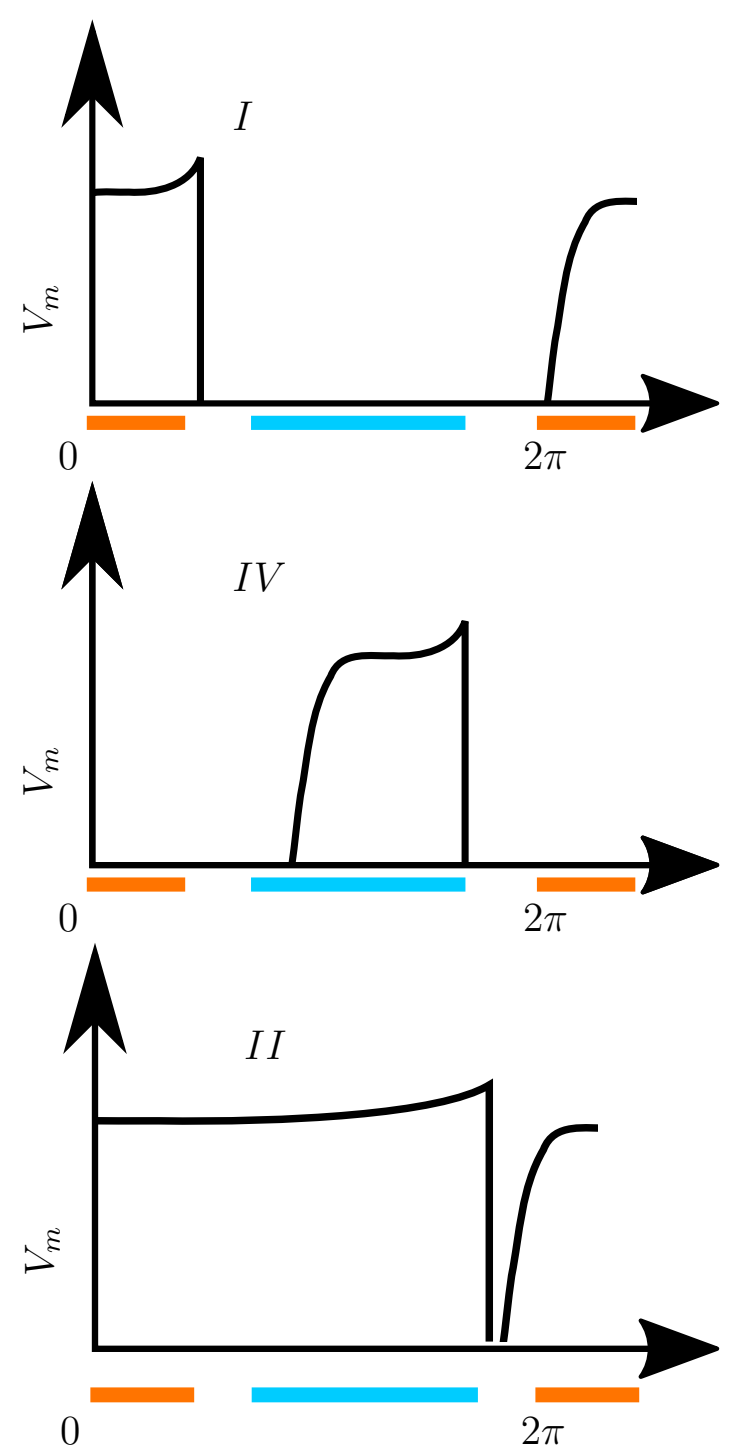

(b)

Figure 9.27.: Alternative Phase Transition Curve - Constructing a new axiomatic model using the effects described in figure 9.28 and assuming that the stimulus is too weak to cause unpinning by the vulnerable window mechanism creates a PTC that explains the observed behavior.

a): The new PTC has again three regions, labeled I, II and IV in this case. Labels have been chosen in such a way as to match figure 3.14. $\mathbf{I}$ is again a subset of the identify, II is again the termination window. Region IV originates from the temporal speedup in the hyper polarized zone.

b) Typical situations that arise in the annotated regions.

I The action potential is on top of the cathode, nothing happens.

IV The action potential is on in the region of the anode and experiences a speedup.

II The speedup causes the wave to hit its own tail and unpin. 


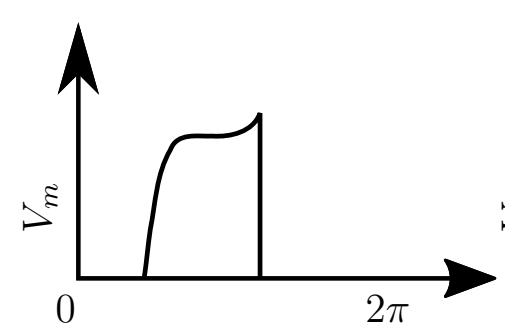

(a)

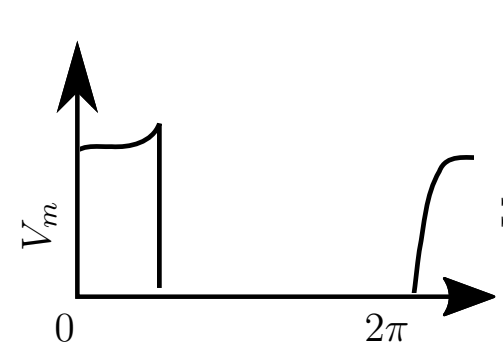

(d)

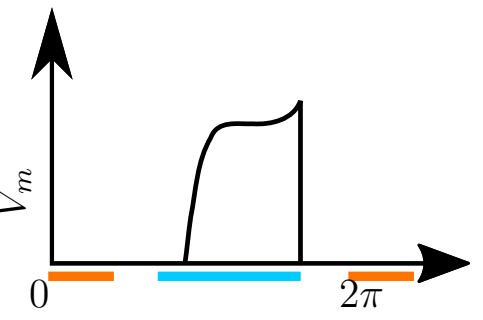

(b)

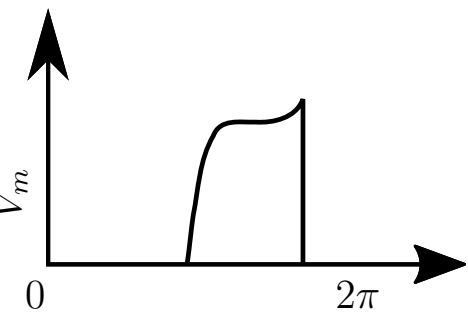

(c)

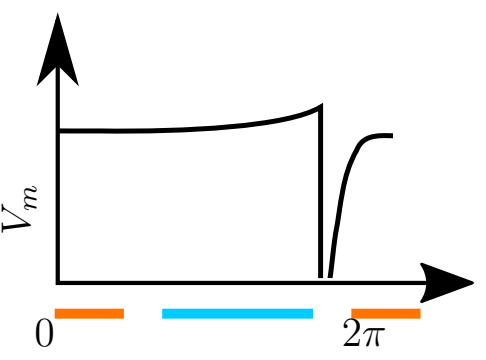

(e)

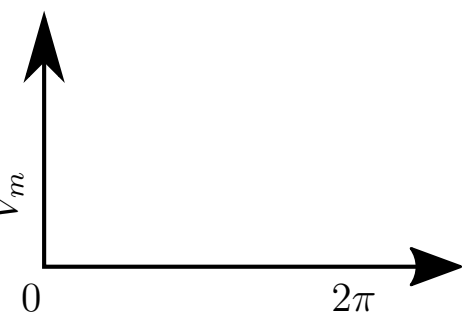

(f)

Figure 9.28.: Acceleration by Hyper Polarization - The pinning center is assumed to be just slightly larger than the apt for this illustrations.

a): At time of shock the action potential still covers the cathode.

b): During the shock and directly after it, the wave is accelerated in the hyper polarized region that forms at the virtual anode.

c): This causes an apparent jump in phase, one period later.

For very small pinning centers the wave front might collide with its tail ( $d$ - e), leading to local conduction block and unpinning.

Implications The phase transition of ventricular tachycardia in response to electric fields has not been investigated before in whole heart experiments. As a consequence the observed is difficult to interpret with respect to its importance. Especially given that of all performed experiments only one allowed to investigate the topic and within this experiment only a small part of the data is usable for this purpose.

From a purely scientific point of view it is interesting that the possibility of a phase increase in response to shock has not been considered before. This might be due to the combination of two unfortunate situations. Firstly simple computational models of excitable media (such as the Barkley model ([12]) used in [14]) do not reproduce the experimentally observed speedup in hyperpolarized tissue. Future numerical studies should be aware of this possibility and use more realistic models of cardiac tissue. Secondly the obstacles used to model pinned waves in cell cultures ([114] or [30] were large compared to the wavelength. This could mask a brief increase of conduction velocity, since the additional distance the wave would travel during this time would be small compared to the obstacles circumference, and hence the increase in phase would also 
be small. The observations made in figure 9.26 are hence important, although they are based on limited data, since they report a possible new effect.

Furthermore the existence of this additional type of phase transition curve in arrhythmias would console the conflicting observation made in the past using periodic pulses for defibrillation, with some authors reporting in favor of periods shorter than the arrhythmia (overdrive pacing see e.g. [74] or [69]) and some reporting in favor of periods longer than the arrhythmia cycle length (underdrive pacing see [96] and [54]). The origin for this might be the different stability of the fixpoints that can arise depending on the PTC and is displayed in figures 9.29 and 9.30. Pacing slower than the arrhythmia avoids a fixpoint in the transition curve described in this section as well as in the one introduced in section 3.6.1. Using a faster frequency however will result in a stable fixpoint in one of the curves and in an unstable one in the other, meaning that phase scanning only works in one of the systems when using overdrive pacing. Assuming that both curves can occur, one would sometimes observe that overdrive pacing fails and sometimes observe it to be superior due to the additional ATP effect.

Looking at the results from a more clinical perspective it is important to note that the unpinning motivated defibrillation methods mentioned in section 4.3 .2 all depend to some degree on the shape of phase transition curves of the assumed pinned spirals. Recall that of the three methods, two ${ }^{7}$ assume the existence of a stable fixpoint in the iterated map construction (see section 3.6.2), whereas the third ${ }^{8}$ implicitly assumes its absence. If the PTC has a form similar to the one displayed in 9.27 there is no stable fixpoint, since an intersection of the rising branch of the curve in region IV would have a derivative greater than 1 and therefore be unstable (see figures 9.29 and 9.30). Future investigation that clarify which of these phase transition curves is more common in a clinicall setting could help to determine which of the methods is superior.

\footnotetext{
${ }^{7}$ LEAP and Synchrolation

${ }^{8}$ Multistage Electrotherapy
} 


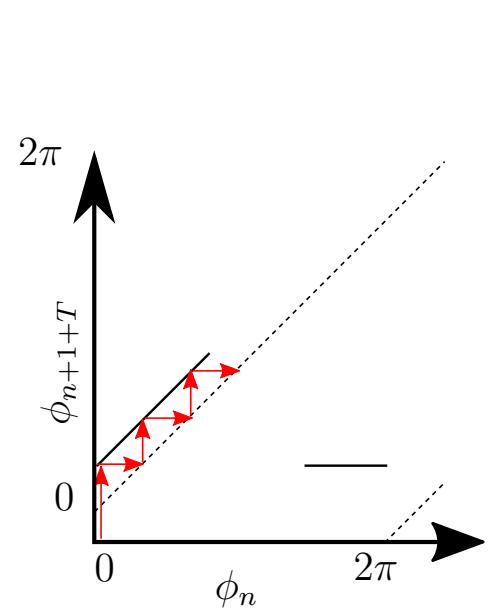

(a)

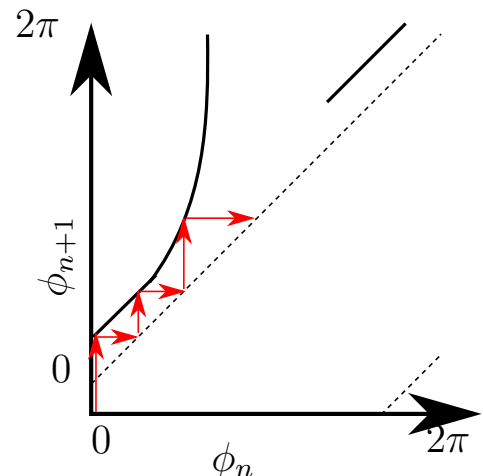

(c)

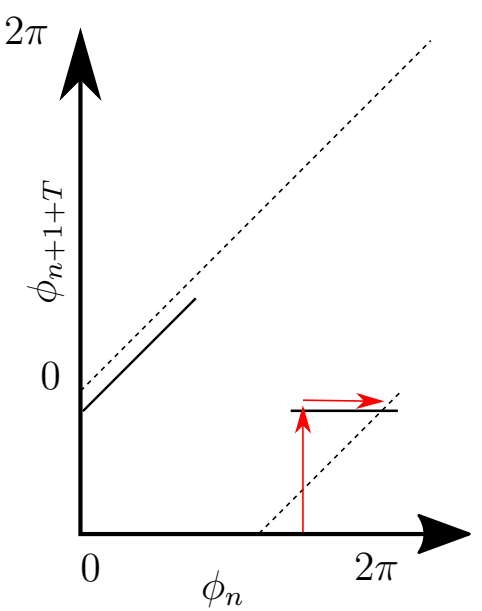

(b)

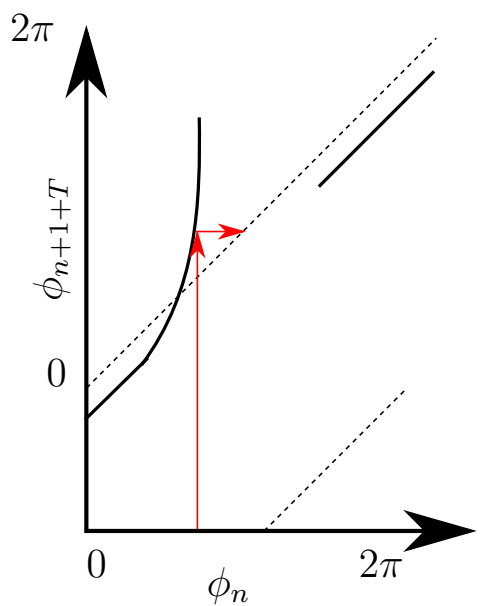

(d)

Figure 9.29.: Over and Underdrive Defibrillation - For multiple periodic shocks the phases at the shocks can be constructed from the shifted PTC (see section 3.6.2 for more details on the construction method). Pacing faster than the arrhythmia frequency $\left(f_{a}\right)$ shifts the PTC downwards, pacing slower upwards. Phases at the time of shock can be read of the cobweb where the red arrows touch the curve. Recall that periodic boundary conditions apply to both axes. The identity is shown as perforated line.

First Column (a,c), Underdrive Defibrillation: For multiple shocks given at a frequency $f<f_{a}$ both PTCs are shifted upwards, no intersection with the identity occurs, phase scanning can be reliably achieved. If the arrhythmia terminates depends on the behavior of the spiral after unpinning. In both cases the spiral is unpinned by the fourth shock

Second Column $(b, d)$ Overdrive Defibrillation: As $f>f_{a}$ both curves are shifted downwards. This leads to intersections with the identity and consequently fixpoints for both curves. However fixpoint in $b$ is stable while the fixpoint in $c$ is not, as can be seen by following the dynamics close to the fixpoints (see figure 9.30 for more a detailed construction around the fixpoint). In case of $c$ overdrive pacing might have the benefit to act as additional ATP after unpinning. 


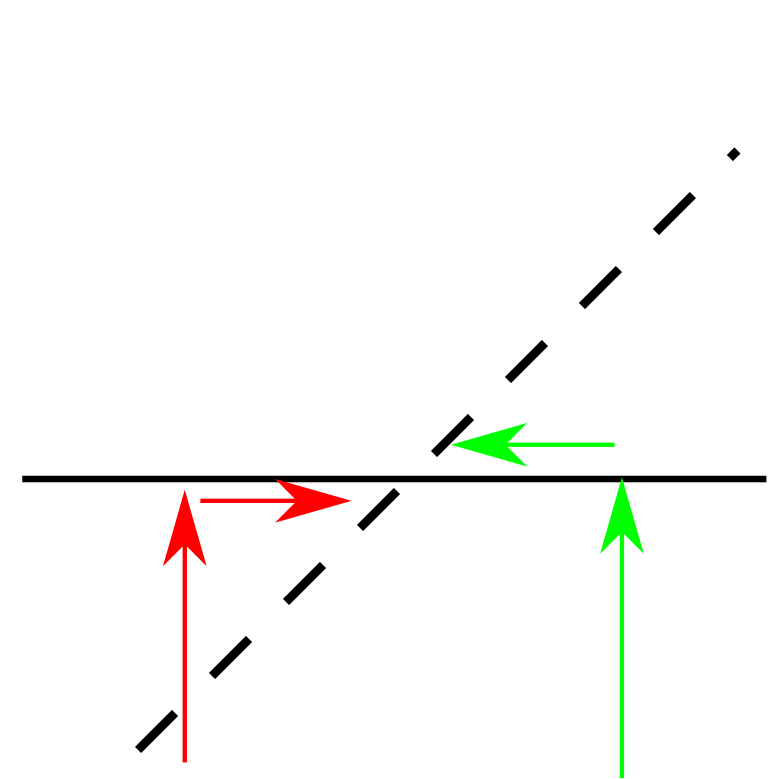

(a)

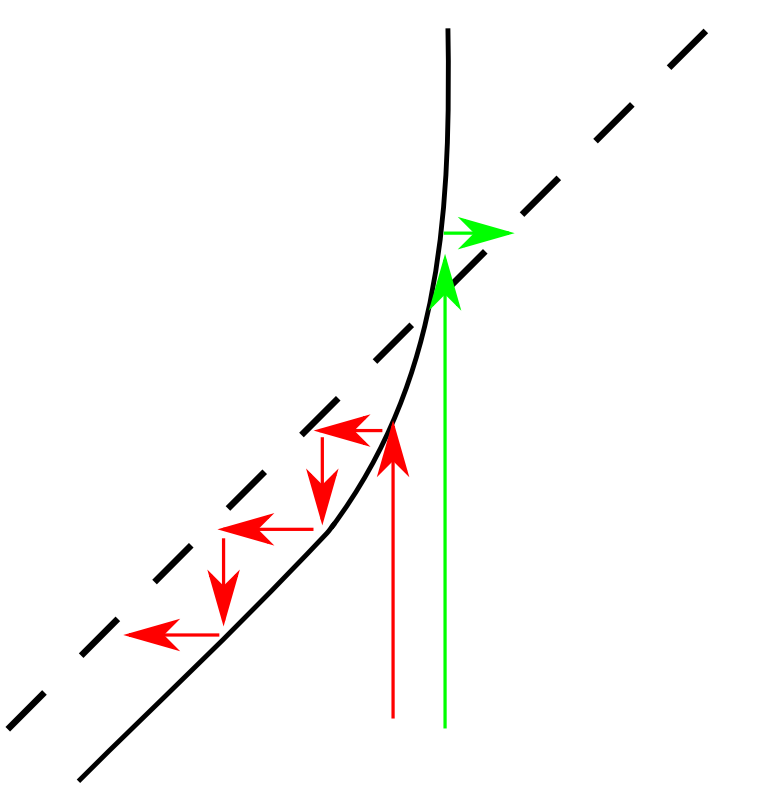

(b)

Figure 9.30.: Cobweb Diagrams for the Dynamics around the Fixpoints - Blow up of the PTCs shown in figure 9.29 around the fixpoints that arise for overdrive defibrillation. Two different sequences of shocks one starting right of the fixpoint (green) one starting left of the fixpoint (red)are shown.

$a$ : The cobweb converges towards the fixpoint for starting phases left and right of it, the fixpoint is therefore attracting and stable. This prevents phase scanning.

$b$ : The cobweb diverges, the fixpoint is unstable. Unless the starting phase is chosen exactly to be the phase of the fixpoint the system will not reach it. 



\section{Summary and Outlook}

\subsection{Summary}

In this document the influence of large heterogeneities on the behavior of ventricular tachycardia under electric field shocks was studied for six different animal models. To facilitate this study a new method to quantify the distance between VT episodes was developed and used to identify a proximity structure between tachycardias. Using this structure it was than possible to objectively evaluate how well different tachycardic states could be reproduced and how stable they were under shock.

For stable, reproducible tachycardia the phase transition was investigated and a hitherto unreported form of phase transition curve was found. Finally the possible implications of this finding were discussed.

Each of this points shall be recalled here in brief to give the reader a coherent overview.

\subsubsection{Distance Measure}

A novel method to measure the distance between VT episodes was defined in section 9.3. It is based on the observation that tachycardia is a low dimensional state ans as such can be characterized using methods developed for low dimensional chaotic systems.

Via this procedure it is possible to objectively identify different tachycardia morphologies. This is done by clustering the morphologies based on the obtained distances (section 9.3.4.

Further more one can evaluate how strongly the individual VT episodes changed due to shock, by measuring the distance between an episode directly before and after shocking (section 9.4.1). 


\subsubsection{Animal Models}

Acute ischemia, reperfused ischemia, cryoablation and chronic myocardial infarction were investigated in pig and rabbit hearts (section 9.1) and it was found that ventricular tachycardia could be induced in cryoablation in rabbits $\left(N_{V T}=4\right.$ out of $N_{\text {total }}=15$ experiments) as well as in acute ischemia $\left(N_{V T}=1\right.$ out of $\left.N_{\text {total }}=1\right)$ and infarction in pigs $\left(N_{V T}=1\right.$ out of $\left.N_{\text {total }}=6\right)$.

Using the affore mentioned distance measure, these models were compared. It was found that the only model system that showed stable reproducible ventricular tachycardia was the infarction model (section 9.5.4). Further more it was found that in the investigated model systems tachycardia was less stable in rabbit hearts than in pig heart (section 9.4.1).

\subsubsection{Phase Transition}

Phase dependence of cardioversion and phase transition behavior was investigated in the one myocardial infarction experiment that allowed for multiple reinductions of the same VT morphologies. Two morphologies showed significant phase dependence as well as well defined phase transition curves. Both were resistant to ATP and driven by a stable focus. For one of these morphologies the PTC could not be explained by the existing theory of a pinned spiral wave and a modification of this theory that could explain the observation was suggested (section 9.6.2).

\subsection{Outlook}

Two main points of this thesis lend themselves to further investigation: Searching for structure in arrhythmias and phase transition curves.

Firstly, the new type of phase transition curve proposed in section 9.6 .2 is highly interesting as it could possibly resolve the standing question if overdrive or underdrive defibrillation is the better candidate for future research. Contrary to PTC reported for cell cultures the new PTC does not predict a higher success rate for under drive methods. As a first step it should be clarified if the proposed shape of a PTC can be reproduced numerically by incorporating the conjectured mechanism into simulations. Should this be the case more experiments will be necessary to clarify the matter further.

Secondly, as mentioned before we recently observed fluctuations in the complexity of the ECG signal ([10]). The search for these fluctuations was partially motivated by the 
observation that the overall irregular ECG signal during fibrillation contains intermittent phases that appear almost VT like. Indeed using simulations we observed that similar fluctuations arise due to the changing number of spiral waves on the heart ([9]). It is hoped that these phases are easier to defibrillate. A further improvement of this approach could be to first identify the low complexity states and than to search for reoccurring morphologies within them, using methods based on the one developed in this document. Should some morphologies be easier to terminate (as it was the case in section 9.6), one could specifically target them for defibrillation.

Finally, clinically the combination of automatic classification and phase dependence of cardioversion could be of relevance. Despite the fact that phase dependence of VT termination has been reported before ([103]), currently triggered cardioversion of VT is still synchronized to the peak of the ECG to avoid VF induction via the vulnerable window mechanism ([72]). This is an eminently sensible approach for most tachycardia episodes. However, for patients suffering from reoccurring ventricular tachycardia a more patient specific approach might be possible.

These individual routinely undergo electrophysiological studies that would allow to measure the phase dependence of VT termination on a per morphology basis. If the patient is equipped with an implanted defibrillator, this defibrillator could be programmed to apply shocks at the optimal phase for those morphologies that were measured during the mentioned studies. 



\section{Bibliography}

[1] European Cardiovascular Disease Statistics 2017 - EHN - European Heart Network, . URL http://www .ehnheart.org/cvd-statistics.html. 1, 31

[2] European Commission : CORDIS : Projects \& Results Service : European Sudden Cardiac Arrest network: towards Prevention, Education and NEw Treatment, . URL http://cordis.europa.eu/project/rcn/207233_en.html. 31

[3] Coronary arteries, May 2017. URL https://en.wikipedia.org/w/index.php? title=Coronary_arteries\&oldid=782545400. Page Version ID: 782545400. 7

[4] Heart, June 2017. URL https://en.wikipedia.org/w/index.php?title= Heart\&oldid=786878815. Page Version ID: 786878815. 6

[5] Jensen-Shannon divergence, May 2017. URL https://en.wikipedia.org/w/ index.php?title=Jensen\%E2\%80\%93Shannon_divergence\&oldid=780000951.

Page Version ID: 780000951. 45

[6] Schlemmer Alexander. Intermittent Complexity Fluctuations during Ventricular Fibrillation. 2

[7] Elad Anter and Mark E. Josephson. 84 - Ischemic Heart Disease. In Douglas P. Zipes and José Jalife, editors, Cardiac Electrophysiology: From Cell to Bedside (Sixth Edition), pages 849-857. W.B. Saunders, Philadelphia, 2014. ISBN 9781-4557-2856-5. URL http://www.sciencedirect.com/science/article/pii/ B9781455728565000844. DOI: 10.1016/B978-1-4557-2856-5.00084-4. 33

[8] Charles Antzelevitch and Alexander Burashnikov. Overview of Basic Mechanisms of Cardiac Arrhythmia. Cardiac electrophysiology clinics, 3(1):23-45, March 2011. ISSN 1877-9182. doi: 10.1016/j.ccep.2010.10.012. URL http://www.ncbi.nlm. nih.gov/pmc/articles/PMC3164530/, 16

[9] S.Luther D.Hornung T.Baig S.Berg E.Boccia U.Parlitz A.Schlemmer, T.Lilienkamp. Unveiling Spatio-Temporal Complexity Fluctuations in Cardiac Arrhythmia Using Permutation Entropy of ECG Time Series. Proceedings of the National Academy of Sciences, . 119 
[10] S.Luther U.Parlitz A.Schlemmer, T.Baig. Detection and characterization of intermittent complexity variations in cardiac arrhythmia. . 69, 118

[11] D \{Barkley\}. A model for fast computer simulation of waves in excitable media. Physica D Nonlinear Phenomena, 49:61-70, April 1991. doi: 10. 1016/0167-2789(91)90194-e. URL http://dx.doi.org/10.1016/0167-2789(91) 90194-e. 11

[12] Dwight Barkley. Barkley model. Scholarpedia, 3(11):1877, November 2008. ISSN 1941-6016. doi: 10.4249/scholarpedia.1877. URL http://www.scholarpedia. org/article/Barkley_model. 112

[13] A. J. Barsky. Palpitations, arrhythmias, and awareness of cardiac activity. Annals of Internal Medicine, 134(9 Pt 2):832-837, May 2001. ISSN 0003-4819. 1

[14] A. Behrend, P. Bittihn, and S. Luther. Predicting unpinning success rates for a pinned spiral in an excitable medium. In 2010 Computing in Cardiology, pages 345-348, September 2010. 23, 24, 26, 30, 34, 35, 106, 112

[15] Vadim N. Biktashev. Resonance and Feedback Strategies for Low-Voltage Defibrillation. In Igor R. Efimov, Mark W. Kroll, and Patrick J. Tchou MD, editors, Cardiac Bioelectric Therapy, pages 493-510. Springer US, 2009. ISBN 978-0387-79402-0 978-0-387-79403-7. URL http://link. springer.com/chapter/10. 1007/978-0-387-79403-7_20. DOI: 10.1007/978-0-387-79403-7_20. 21, 35

[16] Philip Bittihn, Amgad Squires, Gisa Luther, Eberhard Bodenschatz, Valentin Krinsky, Ulrich Parlitz, and Stefan Luther. Phase-resolved analysis of the susceptibility of pinned spiral waves to far-field pacing in a two-dimensional model of excitable media. Philosophical transactions. Series A, Mathematical, physical, and engineering sciences, 368(1918):2221-2236, May 2010. ISSN 1364-503X. doi: 10.1098/rsta.2010.0038. URL http://www.ncbi.nlm.nih.gov/pmc/articles/ PMC2944386/, 26, 34

[17] E. Boccia, S. Luther, and U. Parlitz. Modelling far field pacing for terminating spiral waves pinned to ischaemic heterogeneities in cardiac tissue. Phil. Trans. R. Soc. A, 375(2096):20160289, June 2017. ISSN 1364-503X, 1471-2962. doi: 10.1098/rsta.2016.0289. URL http://rsta.royalsocietypublishing.org/ content/375/2096/20160289. 40

[18] Peter J. A. Bollen, Axel K. Hansen, and Aage K. Olsen Alstrup. The Laboratory Swine, Second Edition. CRC Press, February 2010. ISBN 978-1-4398-1530-4. 56

[19] Ingwer Borg and Patrick JF Groenen. Modern multidimensional scaling: Theory and applications. Springer Science \& Business Media, 2005. 85 
[20] David W. Bourn, Mary M. Maleckar, Blanca Rodriguez, and Natalia A. Trayanova. Mechanistic enquiry into the effect of increased pacing rate on the upper limit of vulnerability. Philosophical Transactions of the Royal Society of London A: Mathematical, Physical and Engineering Sciences, 364(1843):1333-1348, June 2006. ISSN 1364-503X, 1471-2962. doi: 10.1098/rsta.2006.1775. URL http: //rsta.royalsocietypublishing.org/content/364/1843/1333. 18

[21] Clifton W. Callaway, Lawrence D. Sherman, Vincent N. Mosesso, Thomas J. Dietrich, Eric Holt, and M. Christopher Clarkson. Scaling Exponent Predicts Defibrillation Success for Out-of-Hospital Ventricular Fibrillation Cardiac Arrest. Circulation, 103(12):1656-1661, March 2001. ISSN 0009-7322, 1524-4539. doi: 10.1161/01. CIR.103.12.1656. URL http://circ.ahajournals.org/content/103/12/1656. 69

[22] Marco Canepari, Dejan Zecevic, and Olivier Bernus. Membrane Potential Imaging in the Nervous System and Heart. Springer, August 2015. ISBN 978-3-319-17641-3. Google-Books-ID: 3sFOCgAAQBAJ. 110

[23] Daniel J. Cantillon and Bruce L. Wilkoff. Antitachycardia pacing for reduction of implantable cardioverter-defibrillator shocks. Heart Rhythm, 12(6):13701375, June 2015. ISSN 1547-5271, 1556-3871. doi: 10.1016/j.hrthm.2015. 02.024. URL http://www.heartrhythmjournal.com/article/S1547-5271(15) 00203-9/abstract. 17, 19

[24] T. L. Carroll and J. M. Byers. Grid-based partitioning for comparing attractors. Physical Review E, 93(4):042206, April 2016. doi: 10.1103/PhysRevE.93.042206. URL http://link.aps.org/doi/10.1103/PhysRevE.93.042206. 43

[25] Wayne E. Cascio, Hua Yang, Timothy A. Johnson, Barbara J. Muller-Borer, and John J. Lemasters. Electrical Properties and Conduction in Reperfused Papillary Muscle. Circulation Research, 89(9):807-814, October 2001. ISSN 0009-7330, 1524-4571. doi: 10.1161/hh2101.098612. URL http://circres.ahajournals. org/content/89/9/807, 39, 67

[26] Elizabeth M. Cherry and Flavio H. Fenton. Suppression of alternans and conduction blocks despite steep APD restitution: electrotonic, memory, and conduction velocity restitution effects. American Journal of Physiology - Heart and Circulatory Physiology, 286(6):H2332-H2341, June 2004. ISSN 0363-6135, 15221539. doi: 10.1152/ajpheart.00747.2003. URL http://ajpheart.physiology. org/content/286/6/H2332. 32

[27] A. Connolly, P. Gawenda, G. Plank, and M. J. Bishop. Modulation of effective refractory period at the infarct border-zone provides a mechanism for focal arrhythmogenesis. In 2016 Computing in Cardiology Conference (CinC), pages 
929-932, September 2016. 37

[28] James Coromilas, Adam E. Saltman, Bernd Waldecker, Stephen M. Dillon, and Andrew L. Wit. Electrophysiological Effects of Flecainide on Anisotropic Conduction and Reentry in Infarcted Canine Hearts. Circulation, 91(8):2245-2263, April 1995. ISSN 0009-7322, 1524-4539. doi: 10.1161/01.CIR.91.8.2245. URL http://circ.ahajournals.org/content/91/8/2245, 54,64

[29] Joshua Cysyk. Electric field perturbations of pinned reentrant arrhythmias. Ph.D., The Johns Hopkins University, United States - Maryland, 2009. URL http://search.proquest.com/docview/304903114/abstract/ D01247F866C34B9DPQ/1. 35

[30] Joshua Cysyk and Leslie Tung. Electric Field Perturbations of Spiral Waves Attached to Millimeter-Size Obstacles. Biophysical Journal, 94(4):1533-1541, February 2008. ISSN 0006-3495. doi: 10.1529/biophysj.107.116244. URL http: //www.sciencedirect.com/science/article/pii/S0006349508706684, 21, 26, 112

[31] J. M. Davidenko. Spiral wave activity: a possible common mechanism for polymorphic and monomorphic ventricular tachycardias. Journal of Cardiovascular Electrophysiology, 4(6):730-746, December 1993. ISSN 1045-3873. 33

[32] Stefan Dhein, Friedrich Wilhelm Mohr, and Mario Delmar, editors. Practical Methods in Cardiovascular Research. Springer Berlin Heidelberg, Berlin, Heidelberg, 2005. ISBN 978-3-540-40763-8 978-3-540-26574-0. URL http://link.springer. $\mathrm{com} / 10.1007 / \mathrm{b} 137833$. 57

[33] Sanjay Dixit, Edward P. Gerstenfeld, David J. Callans, and Francis E. Marchlinski. Electrocardiographic Patterns of Superior Right Ventricular Outflow Tract Tachycardias: Distinguishing Septal and Free-Wall Sites of Origin. Journal of Cardiovascular Electrophysiology, 14(1):1-7, January 2003. ISSN 1540-8167. doi: 10.1046/j.1540-8167.2003.02404.x. URL http://onlinelibrary.wiley. com/doi/10.1046/j.1540-8167.2003.02404.x/abstract. 33

[34] Derek J. Dosdall and Raymond E. Ideker. 48 - Mechanisms of Ventricular Tachycardia and Fibrillation. In Douglas P. Zipes and José Jalife, editors, Cardiac Electrophysiology: From Cell to Bedside (Sixth Edition), pages 475-482. W.B. Saunders, Philadelphia, 2014. ISBN 978-1-4557-2856-5. URL http:// www.sciencedirect.com/science/article/pii/B9781455728565000480. DOI: 10.1016/B978-1-4557-2856-5.00048-0. 31, 33

[35] J. Délèze. The recovery of resting potential and input resistance in sheep heart injured by knife or laser. The Journal of Physiology, 208(3):547-562, July 1970. 
ISSN 0022-3751. 39

[36] Igor R. Efimov, Mark W. Kroll, and Patrick J. Tchou, editors. Cardiac Bioelectric Therapy. Springer US, Boston, MA, 2009. ISBN 978-0-387-79402-0 978-0-38779403-7. URL http://link.springer.com/10.1007/978-0-387-79403-7. 14. 31,32

[37] IR Efimov, V Sidorov, Y Cheng, and B Wollenzier. Evidence of three-dimensional scroll waves with ribbon-shaped filament as a mechanism of ventricular tachycardia in the isolated rabbit heart. Journal of cardiovascular electrophysiology, 10(11): 1452-1462, November 1999. ISSN 1045-3873. URL http://view.ncbi.nlm.nih. gov/pubmed/10571365. 16

[38] Michael Eldar, Dan Ohad, Amir Bor, Nira Varda-Bloom, David K. Swanson, and Alexander Battler. A Closed-Chest Pig Model of Sustained Ventricular Tachycardia. Pacing and Clinical Electrophysiology, 17(10):1603-1609, October 1994. ISSN 1540-8159. doi: 10.1111/j.1540-8159.1994.tb02353. x. URL http://onlinelibrary.wiley.com/doi/10.1111/j.1540-8159.1994. tb02353.x/abstract. 54

[39] D. M. Endres and J. E. Schindelin. A new metric for probability distributions. IEEE Transactions on Information Theory, 49(7):1858-1860, July 2003. ISSN 0018-9448. doi: 10.1109/TIT.2003.813506. 44

[40] Martin Ester, Hans-Peter Kriegel, Jörg Sander, Xiaowei Xu, and others. A densitybased algorithm for discovering clusters in large spatial databases with noise. In $K d d$, volume 96 , pages $226-231,1996.49,50,73$

[41] Flavio Fenton and Elizabeth Cherry. Models of cardiac cell. Scholarpedia, 3(8): 1868, 2008. ISSN 1941-6016. doi: 10.4249/scholarpedia.1868. URL http://www. scholarpedia.org/article/Models_of_cardiac_cell. 11

[42] Flavio Fenton, Elizabeth Cherry, Harold Hastings, and Steven Evans. Multiple mechanisms of spiral wave breakup in a model of cardiac electrical activity. Chaos: An Interdisciplinary Journal of Nonlinear Science, 12(3):852-892, April 2002. ISSN 10541500. doi: 10.1063/1.1504242. URL http://dx.doi.org/10. 1063/1.1504242. 16

[43] O. Friedrich, F. V Wegner, M. Wink, and R. H. A. Fink. NA+- and K+-channels as molecular targets of the alkaloid ajmaline in skeletal muscle fibres. British Journal of Pharmacology, 151(1):82-93, May 2007. ISSN 0007-1188. doi: 10.1038/ sj.bjp.0707194. 64

[44] D. Gao and J. K. Critser. Mechanisms of cryoinjury in living cells. ILAR journal 
/ National Research Council, Institute of Laboratory Animal Resources, 41(4): 187-196, 2000. ISSN 1084-2020. 38

[45] A Garfinkel, P S Chen, D O Walter, H S Karagueuzian, B Kogan, S J Evans, M Karpoukhin, C Hwang, T Uchida, M Gotoh, O Nwasokwa, P Sager, and J N Weiss. Quasiperiodicity and chaos in cardiac fibrillation. Journal of Clinical Investigation, 99(2):305-314, January 1997. ISSN 0021-9738. URL http: //www.ncbi.nlm.nih.gov/pmc/articles/PMC507798/, 31, 53

[46] null Glass and null Josephson. Resetting and Annihilation of Reentrant Abnormally Rapid Heartbeat. Physical Review Letters, 75(10):2059-2062, September 1995. ISSN 1079-7114. doi: 10.1103/PhysRevLett.75.2059. 23

[47] Richard A. Gray. 34 - Theory of Rotors and Arrhythmias. In Douglas P. Zipes and José Jalife, editors, Cardiac Electrophysiology: From Cell to Bedside (Sixth Edition), pages 341-350. W.B. Saunders, Philadelphia, 2014. ISBN 9781-4557-2856-5. URL http://www.sciencedirect.com/science/article/pii/ B9781455728565000340. DOI: 10.1016/B978-1-4557-2856-5.00034-0. 32

[48] Joris R. de Groot and Ruben Coronel. Acute ischemia-induced gap junctional uncoupling and arrhythmogenesis. Cardiovascular Research, 62(2):323-334, May 2004. ISSN 0008-6363, 1755-3245. doi: 10.1016/j.cardiores.2004.01.033. URL http://cardiovascres.oxfordjournals.org/content/62/2/323. 7

[49] T. Baig H. tom Wörden, D. Hornung. Apparatus for and method of terminating a high frequnecy arrhytmic electric state of biological tissue by timed pulses., 2017. 2. 36

[50] Alexander J. Hill and Paul A. Iaizzo. Comparative Cardiac Anatomy. In Paul A. Iaizzo, editor, Handbook of Cardiac Anatomy, Physiology, and Devices, pages 87-108. Humana Press, 2009. ISBN 978-1-60327-371-8 978-1-60327-3725. URL http://link. springer .com/chapter/10.1007/978-1-60327-372-5_6. DOI: $10.1007 / 978-1-60327-372-5 \_6$. 6]

[51] Art Hobson. Concepts in Statistical Mechanics. CRC Press, 1971. ISBN 978-0677-21870-0. Google-Books-ID: MGIL1NdGNkgC. 43, 44

[52] Nathan E Hoffmann and John C Bischof. The cryobiology of cryosurgical injury. Urology, 60(2, Supplement 1):40-49, August 2002. ISSN 0090-4295. doi: 10. 1016/S0090-4295(02)01683-7. URL http://www.sciencedirect.com/science/ article/pii/S0090429502016837. 39

[53] D. Hornung, V. N. Biktashev, N. F. Otani, T. K. Shajahan, T. Baig, S. Berg, S. Han, V. I. Krinsky, and S. Luther. Mechanisms of vortices termination in the 
cardiac muscle. Royal Society Open Science, 4(3), March 2017. ISSN 2054-5703. doi: 10.1098/rsos.170024. URL http://www.ncbi.nlm.nih.gov/pmc/articles/ PMC5383855/. 24, 26

[54] Daniel Hornung. Cardiac Arrhythmia Termination on the Vascular and Organ Scale. November 2014. URL https://ediss.uni-goettingen.de/handle/ 11858/00-1735-0000-0023-9934-8, 36, 57, 59, 113

[55] Peng-Wie Hsia and Rehan Mahmud. Genesis of Sigmoidal Dose-Response Curve During Defibrillation by Random Shock: A Theoretical Model Based on Experimental Evidence for a Vulnerable Window During Ventricular Fibrillation. Pacing and Clinical Electrophysiology, 13(10):1326-1342, October 1990. ISSN 1540-8159. doi: 10.1111/j.1540-8159.1990.tb02031.x. URL http://onlinelibrary.wiley. com/doi/10.1111/j.1540-8159.1990.tb02031.x/abstract. 34

[56] Iaizzo and A Paul. Handbook of Cardiac Anatomy, Physiology, and Devices. Journal of Cardiac Surgery, 21(3):327-327, June 2006. ISSN 0886-0440. doi: 10.1111/j.1540-8191.2006.00242.x. URL http://dx.doi.org/10.1111/j. $1540-8191.2006 .00242 . x$. 34

[57] Ajit H. Janardhan, Wenwen Li, Vadim V. Fedorov, Michael Yeung, Michael J. Wallendorf, Richard B. Schuessler, and Igor R. Efimov. A Novel Low-Energy Electrotherapy That Terminates Ventricular Tachycardia With Lower Energy Than a Biphasic Shock When Antitachycardia Pacing Fails. Journal of the American College of Cardiology, 60(23):2393-2398, December 2012. ISSN 0735-1097. doi: 10.1016/j.jacc.2012.08.1001. URL http://dx.doi.org/10.1016/j.jacc.2012. 08.1001. 36

[58] Irena Jekova and Vessela Krasteva. Real time detection of ventricular fibrillation and tachycardia. Physiological Measurement, 25(5):1167, 2004. ISSN 0967-3334. doi: 10.1088/0967-3334/25/5/007. URL http://stacks.iop.org/0967-3334/ $25 / i=5 / a=007$. 69

[59] Eric Jones, Travis Oliphant, Pearu Peterson, and others. SciPy: Open source scientific tools for Python. 2001. URL http://www.scipy.org/. 85, 105

[60] Boyoung Joung, Zhengzhe Xu, Ilkwon Kim, Moon-hyoung Lee, and Sungsoon Kim. The Effect of Cryoinjury on Ventricular Tachycardia in the Swine Right Ventricle. Yonsei Medical Journal, 47(5):672-679, October 2006. ISSN 05135796. doi: 10.3349/ymj.2006.47.5.672. URL http://www.ncbi.nlm.nih.gov/ pmc/articles/PMC2687753/. 38, 39, 54, 87

[61] Shiro Kamakura, Wataru Shimizu, Kiyotaka Matsuo, Atsushi Taguchi, Kazuhiro Suyama, Takashi Kurita, Naohiko Aihara, Tohru Ohe, and Katsuro Shimomura. 
Localization of Optimal Ablation Site of Idiopathic Ventricular Tachycardia from Right and Left Ventricular Outflow Tract by Body Surface ECG. Circulation, 98 (15):1525-1533, October 1998. ISSN 0009-7322, 1524-4539. doi: 10.1161/01.CIR. 98.15.1525. URL http://circ.ahajournals.org/content/98/15/1525. 33

[62] Holger Kantz and Thomas Schreiber. Nonlinear time series analysis. Cambridge University Press, January 2004. ISBN 978-0-521-529020. URL http://www . amazon.ca/exec/obidos/redirect?tag=citeulike09-20\& path=ASIN/0521529026. 43, 70

[63] Rie Kato and Pierre Foëx. Myocardial protection by anesthetic agents against ischemia-reperfusion injury: an update for anesthesiologists. Canadian Journal of Anaesthesia = Journal Canadien D'anesthesie, 49(8):777-791, October 2002. ISSN 0832-610X. doi: 10.1007/BF03017409. 103

[64] RE Kerber, NG Pandian, R Hoyt, SR Jensen, S Koyanagi, J Grayzel, and R Kieso. Effect of ischemia, hypertrophy, hypoxia, acidosis, and alkalosis on canine defibrillation. American Journal of Physiology - Heart and Circulatory Physiology, 244(6): H825-H831, June 1983. ISSN 1522-1539. URL http://ajpheart.physiology. org/content/244/6/H825. abstract. 39

[65] Stephen B. Knisley, Robert K. Justice, Wei Kong, and Philip L. Johnson. Ratiometry of transmembrane voltage-sensitive fluorescent dye emission in hearts. American Journal of Physiology - Heart and Circulatory Physiology, 279(3):H1421H1433, September 2000. ISSN 0363-6135, 1522-1539. URL http://ajpheart. physiology.org/content/279/3/H1421. 57

[66] VI Krinsky. Fibrillation in excitable media. Cybernetics problems, 20:59-80, 1968. 16

[67] Pawel Kuklik, Prashanthan Sanders, Lukasz Szumowski, and Jan J. Żebrowski. Attraction and repulsion of spiral waves by inhomogeneity of conduction anisotropy - a model of spiral wave interaction with electrical remodeling of heart tissue. Journal of Biological Physics, 39(1):67-80, January 2013. ISSN 00920606. doi: 10.1007/s10867-012-9286-4. URL http://www.ncbi.nlm.nih.gov/ pmc/articles/PMC3532668/. 19, 35

[68] Pavlos P Lelovas, Nikolaos G Kostomitsopoulos, and Theodoros T Xanthos. A Comparative Anatomic and Physiologic Overview of the Porcine Heart. Journal of the American Association for Laboratory Animal Science : JAALAS, 53(5): 432-438, September 2014. ISSN 1559-6109. URL http://www.ncbi.nlm.nih. gov/pmc/articles/PMC4181683/, 6

[69] Wenwen Li, Crystal M. Ripplinger, Qing Lou, and Igor R. Efimov. Multiple 
Monophasic Shocks Improve Electrotherapy of Ventricular Tachycardia in a Rabbit Model of Chronic Infarction. Heart rhythm : the official journal of the Heart Rhythm Society, 6(7):1020, July 2009. ISSN 1547-5271. doi: 10.1016/j.hrthm.2009. 03.015. URL http://www.ncbi.nlm.nih.gov/pmc/articles/PMC2813769/. 54, 64, 69, 113

[70] C. C. Lin and Y. Y. Lau. Density Wave Theory of Spiral Structure of Galaxies. Studies in Applied Mathematics, 60(2):97-163, April 1979. ISSN 1467-9590. doi: 10.1002/sapm197960297. URL http://onlinelibrary.wiley . com/doi/10. 1002/sapm197960297/abstract. 15

[71] Mark S. Link, Paul J. Wang, Natesa G. Pandian, Saroja Bharati, James E. Udelson, Man-Young Lee, Mark A. Vecchiotti, Brian A. VanderBrink, Gianluca Mirra, Barry J. Maron, and N.A. Mark Estes. An Experimental Model of Sudden Death Due to Low-Energy Chest-Wall Impact (Commotio Cordis). New England Journal of Medicine, 338(25):1805-1811, June 1998. ISSN 00284793. doi: 10.1056/NEJM199806183382504. URL http://dx.doi.org/10.1056/ NEJM199806183382504. 16

[72] Mark S. Link, Dianne L. Atkins, Rod S. Passman, Henry R. Halperin, Ricardo A. Samson, Roger D. White, Michael T. Cudnik, Marc D. Berg, Peter J. Kudenchuk, and Richard E. Kerber. Part 6: Electrical Therapies. Circulation, 122 (18 suppl 3):S706-S719, November 2010. ISSN 0009-7322, 1524-4539. doi: 10. 1161/CIRCULATIONAHA.110.970954. URL http://circ.ahajournals.org/ content/122/18_suppl_3/S706. 119

[73] Mark S. Link, Lauren C. Berkow, Peter J. Kudenchuk, Henry R. Halperin, Erik P. Hess, Vivek K. Moitra, Robert W. Neumar, Brian J. O'Neil, James H. Paxton, Scott M. Silvers, Roger D. White, Demetris Yannopoulos, and Michael W. Donnino. Part 7: Adult Advanced Cardiovascular Life Support. Circulation, 132(18 suppl 2):S444-S464, November 2015. ISSN 0009-7322, 1524-4539. doi: 10.1161/ CIR.0000000000000261. URL http://circ . ahajournals .org/content/132/18_ suppl_2/S444. 53

[74] Stefan Luther, Flavio H. Fenton, Bruce G. Kornreich, Amgad Squires, Philip Bittihn, Daniel Hornung, Markus Zabel, James Flanders, Andrea Gladuli, Luis Campoy, Elizabeth M. Cherry, Gisa Luther, Gerd Hasenfuss, Valentin I. Krinsky, Alain Pumir, Robert F. Gilmour Jr, and Eberhard Bodenschatz. Low-energy control of electrical turbulence in the heart. Nature, 475(7355):235-239, July 2011. ISSN 0028-0836. doi: 10.1038/nature10216. URL https://www . nature.com/nature/ journal/v475/n7355/full/nature10216.html, 2, 32, 36, 113

[75] Robert Malkin, Joseph Souza, and Raymond Ideker. The Ventricular Defibrillation and Upper Limit of Vulnerability Dose-Response Curves. Journal of Cardiovascu- 
lar Electrophysiology, 8(8):895-903, August 1997. doi: 10.1111/j.1540-8167.1997. tb00851.x. URL http://dx.doi.org/10.1111/j.1540-8167.1997.tb00851.x. 34

[76] Nathan Mantel. The Detection of Disease Clustering and a Generalized Regression Approach. Cancer Research, 27(2 Part 1):209-220, February 1967. ISSN 00085472, 1538-7445. URL http://cancerres.aacrjournals.org/content/27/2_ Part_1/209. 49

[77] Kaname Matsumura, Richmond W. Jeremy, Jutta Schaper, and Lewis C. Becker. Progression of Myocardial Necrosis During Reperfusion of Ischemic Myocardium. Circulation, 97(8):795-804, March 1998. ISSN 0009-7322, 1524-4539. doi: 10.1161/ 01.CIR.97.8.795. URL http://circ.ahajournals.org/content/97/8/795, 39

[78] Peter Mccullagh. Resampling and exchangeable arrays. Bernoulli, 6(2):285301, April 2000. ISSN 1350-7265. URL http://projecteuclid.org/euclid. bj/1081788029. 69

[79] John H McDonald. Handbook of biological statistics, volume 2. Sparky House Publishing Baltimore, MD, 2009. 48

[80] Mark E. Josephson MD. Josephson's Clinical Cardiac Electrophysiology. LWW, Philadelphia, fifth edition edition, September 2015. ISBN 978-1-4511-8741-0. 37. 53, 61, 69, 97

[81] AS Mikhailov and VI Krinsky. Rotating spiral waves in excitable media: The analytical results. Physica D: Nonlinear Phenomena, 9(3):346-371, December 1983. ISSN 01672789. doi: 10.1016/0167-2789(83)90277-4. URL http://dx.doi.org/ 10.1016/0167-2789(83)90277-4. 15

[82] John W. Milnor. Attractor. Scholarpedia, 1(11):1815, November 2006. ISSN 1941-6016. doi: 10.4249/scholarpedia.1815. URL http://www.scholarpedia. org/article/Attractor. 41

[83] Arthur J. Moss, Wojciech Zareba, W. Jackson Hall, Helmut Klein, David J. Wilber, David S. Cannom, James P. Daubert, Steven L. Higgins, Mary W. Brown, and Mark L. Andrews. Prophylactic Implantation of a Defibrillator in Patients with Myocardial Infarction and Reduced Ejection Fraction. New England Journal of Medicine, 346(12):877-883, March 2002. ISSN 0028-4793. doi: 10.1056/NEJMoa013474. URL http://dx.doi.org/10.1056/NEJMoa013474. 2

[84] Robert J. Myerburg, Alberto Interian, Raul M. Mitrani, Kenneth M. Kessler, and Agustin Castellanos. Frequency of Sudden Cardiac Death and Profiles of Risk. American Journal of Cardiology, 80(5):10F-19F, September 1997. ISSN 
0002-9149, 1879-1913. doi: 10.1016/S0002-9149(97)00477-3. URL http://www. ajconline.org/article/S0002-9149(97)00477-3/fulltext, 2, 37

[85] J. C. Neu and W. Krassowska. Homogenization of syncytial tissues. Critical Reviews in Biomedical Engineering, 21(2):137-199, 1993. ISSN 0278-940X. 11

[86] David Newman, Aiala Barr, Mary Greene, David Martin, Miney Ham, Sally Thorne, and Paul Dorian. A Population-Based Method for the Estimation of Defibrillation Energy Requirements in Humans Assessment of Time-Dependent Effects With a Transvenous Defibrillation System. Circulation, 96(1):267-273, July 1997. ISSN 0009-7322, 1524-4539. doi: 10.1161/01.CIR.96.1.267. URL http://circ.ahajournals.org/content/96/1/267. 64

[87] M. I. Owis, A. H. Abou-Zied, A. B. M. Youssef, and Y. M. Kadah. Study of features based on nonlinear dynamical modeling in ECG arrhythmia detection and classification. IEEE Transactions on Biomedical Engineering, 49(7):733-736, July 2002. ISSN 0018-9294. doi: 10.1109/TBME.2002.1010858. 69

[88] D. Pazó, L. Kramer, A. Pumir, S. Kanani, I. Efimov, and V. Krinsky. Pinning Force in Active Media. Physical Review Letters, 93(16):168303, October 2004. doi: 10.1103/PhysRevLett.93.168303. URL https://link.aps.org/doi/ 10.1103/PhysRevLett.93.168303. 19

[89] Fabian Pedregosa, Gaël Varoquaux, Alexandre Gramfort, Vincent Michel, Bertrand Thirion, Olivier Grisel, Mathieu Blondel, Peter Prettenhofer, Ron Weiss, Vincent Dubourg, Jake Vanderplas, Alexandre Passos, David Cournapeau, Matthieu Brucher, Matthieu Perrot, and Édouard Duchesnay. Scikit-learn: Machine Learning in Python. J. Mach. Learn. Res., 12:2825-2830, November 2011. ISSN 1532-4435. URL http://dl.acm.org/citation.cfm?id=1953048. 2078195. 75.

[90] Nicholas S. Peters. Myocardial gap junction organization in ischemia and infarction. Microscopy Research and Technique, 31(5):375-386, August 1995. ISSN 1097-0029. doi: 10.1002/jemt.1070310507. URL http://onlinelibrary.wiley. com/doi/10.1002/jemt.1070310507/abstract. 37

[91] Sven T. Pleger, Changguang Shan, Jan Ksienzyk, Raffi Bekeredjian, Peter Boekstegers, Rabea Hinkel, Stefanie Schinkel, Barbara Leuchs, Jochen Ludwig, Gang Qiu, Christophe Weber, Philip Raake, Walter J. Koch, Hugo A. Katus, Oliver J. Müller, and Patrick Most. Cardiac AAV9-S100a1 Gene Therapy Rescues PostIschemic Heart Failure in a Preclinical Large Animal Model. Science Translational Medicine, 3(92):92ra64-92ra64, July 2011. ISSN 1946-6234, 1946-6242. doi: 10.1126/scitranslmed.3002097. URL http://stm.sciencemag.org/content/3/ 92/92ra64. 61. 
[92] B. Podesser, G. Wollenek, R. Seitelberger, H. Siegel, E. Wolner, W. Firbas, and M. Tschabitscher. Epicardial branches of the coronary arteries and their distribution in the rabbit heart: the rabbit heart as a model of regional ischemia. The Anatomical Record, 247(4):521-527, April 1997. ISSN 0003-276X. 6, 92

[93] Jeanne E. Poole, George W. Johnson, Anne S. Hellkamp, Jill Anderson, David J. Callans, Merritt H. Raitt, Ramakota K. Reddy, Francis E. Marchlinski, Raymond Yee, Thomas Guarnieri, Mario Talajic, David J. Wilber, Daniel P. Fishbein, Douglas L. Packer, Daniel B. Mark, Kerry L. Lee, and Gust H. Bardy. Prognostic Importance of Defibrillator Shocks in Patients with Heart Failure. New England Journal of Medicine, 359(10):1009-1017, September 2008. ISSN 0028-4793. doi: 10.1056/NEJMoa071098. URL http://dx.doi.org/10.1056/NEJMoa071098. 2

[94] Craig M. Pratt and Lemuel A. Moyé. The Cardiac Arrhythmia Suppression Trial. Circulation, 91(1):245-247, January 1995. ISSN 0009-7322, 1524-4539. doi: 10.1161/01.CIR.91.1.245. URL http://circ.ahajournals.org/content/91/1/ 245. 33

[95] Alain Pumir and Valentin Krinsky. Unpinning of a Rotating Wave in Cardiac Muscle by an Electric Field. Journal of Theoretical Biology, 199(3):311319, August 1999. ISSN 0022-5193. doi: 10.1006/jtbi.1999.0957. URL http: //www.sciencedirect.com/science/article/pii/S0022519399909578, 19, 20, 21,35

[96] Lukas J. Rantner, Brock M. Tice, and Natalia A. Trayanova. Terminating Ventricular Tachyarrhythmias Using Far-Field Low-Voltage Stimuli: Mechanisms and Delivery Protocols. Heart rhythm : the official journal of the Heart Rhythm Society, 10(8):1209-1217, August 2013. ISSN 1547-5271. doi: 10.1016/j.hrthm.2013.04.027. URL http://www.ncbi.nlm.nih.gov/pmc/articles/PMC3735828/. 113

[97] Flavia Ravelli and Renzo Antolini. Dimensional Analysis of the Ventricular Fibrillation ECG. In Arun V. Holden, Mario Markus, and Hans G. Othmer, editors, Nonlinear Wave Processes in Excitable Media, number 244 in NATO ASI Series, pages 335-342. Springer US, 1991. ISBN 978-1-4899-3685-1 978-1-4899-36837. URL http://link.springer.com/chapter/10.1007/978-1-4899-3683-7_ 31. DOI: $10.1007 / 978-1-4899-3683-7 \_31$. 53

[98] Antonio Raviele, Franco Giada, Lennart Bergfeldt, Jean Jacques Blanc, Carina Blomstrom-Lundqvist, Lluis Mont, John M. Morgan, M. J. Pekka Raatikainen, Gerhard Steinbeck, Sami Viskin, Paulus Kirchhof, Frieder Braunschweig, Martin Borggrefe, Meleze Hocini, Paolo Della Bella, and Dipen Chandrakant Shah. Management of patients with palpitations: a position paper from the European Heart Rhythm Association. EP Europace, 13(7):920-934, July 2011. ISSN 1099-5129. doi: 10.1093/ 
europace/eur130. URL https://academic.oup.com/europace/article/13/7/ 920/447426/Management-of-patients-with-palpitations-a. 1

[99] S. Reek, C. J. Geller, W. M. Hartung, and H. U. Klein. Tiermodelle für ventrikuläre Tachyarrhythmien. Herzschrittmachertherapie und Elektrophysiologie, 10(1):3041, March 1999. ISSN 0938-7412. doi: 10.1007/s003990050046. URL http: //link.springer.com/article/10.1007/s003990050046. 38

[100] WJ Richardson, SA Clarke, TA Quinn, and JW Holmes. Physiological Implications of Myocardial Scar Structure. Comprehensive Physiology, 5(4):18771909, September 2015. ISSN 2040-4603. doi: 10.1002/cphy.c140067. URL http://www.ncbi.nlm.nih.gov/pmc/articles/PMC4727398/. 37

[101] M. Richter, T. Schreiber, and D. T. Kaplan. Fetal ECG extraction with nonlinear state-space projections. IEEE Transactions on Biomedical Engineering, 45(1): 133-137, January 1998. ISSN 0018-9294. doi: 10.1109/10.650369. 41

[102] Crystal M. Ripplinger and Igor R. Efimov. The Virtual Electrode Hypothesis of Defibrillation. In Igor R. Efimov, Mark W. Kroll, and Patrick J. Tchou MD, editors, Cardiac Bioelectric Therapy, pages 331-356. Springer US, 2009. ISBN 978-0387-79402-0 978-0-387-79403-7. URL http://link. springer.com/chapter/10. 1007/978-0-387-79403-7_13. DOI: 10.1007/978-0-387-79403-7_13. 8, 13, 35

[103] Crystal M. Ripplinger, Valentin I. Krinsky, Vladimir P. Nikolski, and Igor R. Efimov. Mechanisms of unpinning and termination of ventricular tachycardia. American Journal of Physiology - Heart and Circulatory Physiology, 291(1):H184H192, July 2006. ISSN 0363-6135, 1522-1539. doi: 10.1152/ajpheart.01300.2005. URL http://ajpheart.physiology .org/content/291/1/H184. 34, 36, 119

[104] Crystal M. Ripplinger, Qing Lou, Wenwen Li, Jennifer Hadley, and Igor R. Efimov. Panoramic imaging reveals basic mechanisms of induction and termination of ventricular tachycardia in rabbit heart with chronic infarction: implications for low-voltage cardioversion. Heart Rhythm: The Official Journal of the Heart Rhythm Society, 6(1):87-97, January 2009. ISSN 1556-3871. doi: 10.1016/j.hrthm.2008.09.019. 33, 34, 61, 62, 64,

[105] Felice M. Roberts, Richard J. Povinelli, and Kristina M. Ropella. Identification of ECG Arrhythmias Using Phase Space Reconstruction. In Principles of Data Mining and Knowledge Discovery, pages 411-423. Springer, Berlin, Heidelberg, September 2001. doi: 10.1007/3-540-44794-6_34. URL https://link.springer. com/chapter/10.1007/3-540-44794-6_34. 41, 53

[106] Véronique L Roger. Epidemiology of myocardial infarction. The Medical clinics of North America, 91(4):537-ix, July 2007. ISSN 0025-7125. doi: 10.1016/j.mcna. 
2007.03.007. URL http://www.ncbi.nlm.nih.gov/pmc/articles/PMC2537993/. 37

[107] Bradley J. Roth. Bidomain model. Scholarpedia, 3(4):6221, April 2008. ISSN 1941-6016. doi: 10.4249/scholarpedia.6221. URL http://www.scholarpedia. org/article/Bidomain_model. 13, 14

[108] Michael Rubart and Douglas P. Zipes. Mechanisms of sudden cardiac death. The Journal of Clinical Investigation, 115(9):2305-2315, September 2005. ISSN 0021-9738. doi: 10.1172/JCI26381. URL/https://www.jci.org/articles/view/ 26381. 31, 32, 33

[109] Yoram Rudy. The Forward Problem of Electrocardiography Revisited. Circulation: Arrhythmia and Electrophysiology, 8(3):526-528, June 2015. ISSN 1941-3149, 1941-3084. doi: 10.1161/CIRCEP.115.003056. URL http://circep. ahajournals .org/content/8/3/526. 104

[110] Tim Sauer, James A. Yorke, and Martin Casdagli. Embedology. Journal of Statistical Physics, 65(3-4):579-616, November 1991. ISSN 0022-4715, 15729613. doi: 10.1007/BF01053745. URL https://link.springer.com/article/ 10.1007/BF01053745. 41, 70

[111] C. Schmid, J. Nitsch, and B. Lüderitz. Acute induction of sustained ventricular tachycardia: A new porcine model. Research in Experimental Medicine, 189(5): 363-370, September 1989. ISSN 0300-9130, 1433-8580. doi: 10.1007/BF01855042. URL http://link.springer.com/article/10.1007/BF01855042. 40, 54, 64, 104

[112] Bernhard Schölkopf, Alexander Smola, and Klaus-Robert Müller. Kernel principal component analysis. In Artificial Neural Networks - ICANN'97, pages 583-588. Springer, Berlin, Heidelberg, October 1997. doi: 10.1007/BFb0020217. URL https://link.springer.com/chapter/10.1007/BFb0020217. 48

[113] Ronald H. Selvester, David G. Strauss, and Galen S. Wagner. Myocardial Infarction. In Peter W. Macfarlane, A. van Oosterom, Olle Pahlm, Paul Kligfield, Michiel Janse, and John Camm, editors, Comprehensive Electrocardiology, pages 651-746. Springer London, 2010. ISBN 978-1-84882-045-6 978-184882-046-3. URL http://link. springer .com/referenceworkentry/10.1007/ 978-1-84882-046-3_16. DOI: 10.1007/978-1-84882-046-3_16. 37

[114] T. K. Shajahan, Sebastian Berg, Stefan Luther, Valentin Krinski, and Philip Bittihn. Scanning and resetting the phase of a pinned spiral wave using periodic far field pulses. New Journal of Physics, 18(4):043012, 2016. ISSN 1367-2630. doi: 10.1088/1367-2630/18/4/043012. URL http://stacks .iop.org/1367-2630/18/ 
$i=4 / a=043012,21,27,29,106,112$

[115] Sharad Shandilya, Kevin Ward, Michael Kurz, and Kayvan Najarian. Non-linear dynamical signal characterization for prediction of defibrillation success through machine learning. BMC Medical Informatics and Decision Making, 12:116, 2012. ISSN 1472-6947. doi: 10.1186/1472-6947-12-116. URL http://dx.doi.org/10. 1186/1472-6947-12-116. 69

[116] Shi Shu, Xiong Liu, and Edward D. Korn. Blebbistatin and blebbistatininactivated myosin II inhibit myosin II-independent processes in Dictyostelium. Proceedings of the National Academy of Sciences of the United States of America, 102(5):1472-1477, February 2005. ISSN 0027-8424, 1091-6490. doi: 10.1073/pnas. 0409528102. URL http://www.pnas.org/content/102/5/1472, 57

[117] George Silvay, Javier G. Castillo, Joanna Chikwe, Brigid Flynn, and Farzan Filsoufi. Cardiac Anesthesia and Surgery in Geriatric Patients. Seminars in Cardiothoracic and Vascular Anesthesia, 12(1):18-28, March 2008. ISSN 1089-2532. doi: 10.1177/1089253208316446. URL http://journals . sagepub.com/doi/abs/10. 1177/1089253208316446. 53

[118] Joseph Starobin, Yuri I. Zilberter, and C. Frank Starmer. Vulnerability in onedimensional excitable media. Physica D: Nonlinear Phenomena, 70(4):321-341, February 1994. ISSN 0167-2789. doi: 10.1016/0167-2789(94)90069-8. URL http: //wWw.sciencedirect.com/science/article/pii/0167278994900698. 17

[119] David M. Steinhaus, Debbie S. Cardinal, Luc Mongeon, Shailesh Kumar Musley, Laura Foley, and Susie Corrigan. Internal defibrillation: pain perception of low energy shocks. Pacing and clinical electrophysiology: PACE, 25(7):1090-1093, July 2002. ISSN 0147-8389. 31, 53

[120] William G. Stevenson. Ventricular Scars and Ventricular Tachycardia. Transactions of the American Clinical and Climatological Association, 120:403-412, 2009. ISSN 0065-7778. URL http://www.ncbi.nlm.nih.gov/pmc/articles/ PMC2744510/. 33

[121] J. G. Stinstra, S. Shome, B. Hopenfeld, and R. S. MacLeod. Modelling passive cardiac conductivity during ischaemia. Medical $\&$ Biological Engineering $\& 5$ Computing, 43(6):776-782, November 2005. ISSN 0140-0118. 39

[122] Steven Strogatz. Yeast oscillations, belousov-zhabotinsky waves, and the nonretraction theorem. The Mathematical Intelligencer, 7(2):9-17, June 1985. ISSN 0343-6993. doi: 10.1007/bf03024168. URL http://dx.doi.org/10.1007/ bf03024168. 20. 
[123] Hiromichi Suetani, Karin Soejima, Rei Matsuoka, Ulrich Parlitz, and Hiroki Hata. Manifold learning approach for chaos in the dripping faucet. Physical Review E, 86(3):036209, September 2012. doi: 10.1103/PhysRevE.86.036209. URL http: //link.aps.org/doi/10.1103/PhysRevE.86.036209. 43

[124] G. Taimor. Cardiac gap junctions: good or bad? Cardiovascular Research, 48 (1):8-10, October 2000. ISSN 0008-6363, 1755-3245. doi: 10.1016/S0008-6363(00) 00189-9. URL http://cardiovascres.oxfordjournals.org/content/48/1/8. 7. 39

[125] S. Takagi, A. Pumir, D. Pazó, I. Efimov, V. Nikolski, and V. Krinsky. Unpinning and removal of a rotating wave in cardiac muscle. Physical Review Letters, 93(5): 058101, July 2004. ISSN 0031-9007. doi: 10.1103/PhysRevLett.93.058101. 19

[126] Floris Takens. Detecting strange attractors in turbulence. In Dynamical Systems and Turbulence, Warwick 1980, pages 366-381. Springer, Berlin, Heidelberg, 1981. URL https://link.springer.com/chapter/10.1007/BFb0091924. DOI: 10.1007/BFb0091924. 42

[127] John Michael Tutill Thompson and H Bruce Stewart. Nonlinear dynamics and chaos. John Wiley \& Sons, 2002. 30

[128] Natalia A. Trayanova. Models of Arrhythmogenesis in Myocardial Infarction. In XIII Mediterranean Conference on Medical and Biological Engineering and Computing 2013, pages 1859-1863. Springer, Cham, 2014. URL https://link. springer.com/chapter/10.1007/978-3-319-00846-2_458. DOI: $10.1007 / 978$ 3-319-00846-2_458. 37

[129] Cory M. Tschabrunn, Sébastien Roujol, Reza Nezafat, Beverly Faulkner-Jones, Alfred E. Buxton, Mark E. Josephson, and Elad Anter. A swine model of infarctrelated reentrant ventricular tachycardia: Electroanatomic, magnetic resonance, and histopathological characterization. Heart Rhythm, 13(1):262-273, January 2016. ISSN 1556-3871. doi: 10.1016/j.hrthm.2015.07.030. 102, 103

[130] Roderick Tung, Noel G. Boyle, and Kalyanam Shivkumar. CATHETER ABLATION OF VENTRICULAR TACHYCARDIA. Circulation, 122(3):e389-e391, July 2010. ISSN 0009-7322. doi: 10.1161/CIRCULATIONAHA.110.963371. URL http://www.ncbi.nlm.nih.gov/pmc/articles/PMC2918802/, 33, 38

[131] K. H. W. J. ten Tusscher, D. Noble, P. J. Noble, and A. V. Panfilov. A model for human ventricular tissue. American Journal of Physiology - Heart and Circulatory Physiology, 286(4):H1573-H1589, April 2004. ISSN 0363-6135, $1522-$ 1539. doi: 10.1152/ajpheart.00794.2003. URL http://ajpheart.physiology. org/content/286/4/H1573. 11. 
[132] John J. Tyson, Kevin A. Alexander, V. S. Manoranjan, and J. D. Murray. Spiral waves of cyclic amp in a model of slime mold aggregation. Physica D: Nonlinear Phenomena, 34(1):193-207, January 1989. ISSN 0167-2789. doi: 10. 1016/0167-2789(89)90234-0. URL http://www.sciencedirect.com/science/ article/pii/0167278989902340. 16

[133] Miguel Valderrábano, Peng-Sheng Chen, and Shien-Fong Lin. Spatial Distribution of Phase Singularities in Ventricular Fibrillation. Circulation, 108(3):354-359, July 2003. ISSN 1524-4539. doi: 10.1161/01.cir.0000080322.67408.b4. URL http: //dx.doi.org/10.1161/01.cir.0000080322.67408.b4. 2, 53

[134] Johannes B. van Rees, C. Jan Willem Borleffs, Mihály K. de Bie, Theo Stijnen, Lieselot van Erven, Jeroen J. Bax, and Martin J. Schalij. Inappropriate Implantable Cardioverter-Defibrillator Shocks: Incidence, Predictors, and Impact on Mortality. Journal of the American College of Cardiology, 57(5):556-562, February 2011. ISSN 0735-1097. doi: 10.1016/j.jacc.2010.06.059. URL http: //www.sciencedirect.com/science/article/pii/S0735109710045729. 31

[135] Jakob Vinten-Johansen, Amanda J. Zatta, Rong Jiang, and Weiwei Shi. Lethal Myocardial Reperfusion Injury. In Juan Carlos Kaski, Derek J. Hausenloy, Bernard John Gersh, and Derek M. Yellon, editors, Management of Myocardial Reperfusion Injury, pages 51-85. Springer London, 2012. ISBN 978-1-84996018-2 978-1-84996-019-9. URL http://link.springer.com/chapter/10.1007/ 978-1-84996-019-9_4. 39, 40

[136] Peter H Westfall and S Stanley Young. Resampling-based multiple testing: Examples and methods for p-value adjustment, volume 279. John Wiley \& Sons, 1993. 69

[137] A. T. Winfree. The prehistory of the Belousov-Zhabotinsky oscillator. Journal of Chemical Education, 61(8):661, August 1984. ISSN 0021-9584. doi: 10.1021/ ed061p661. URL http://dx.doi.org/10.1021/ed061p661. 15

[138] Arthur T. Winfree. Electrical Singular Filaments in the Heart Wall. In The Geometry of Biological Time, number 12 in Interdisciplinary Applied Mathematics, pages 495-536. Springer New York, 2001. ISBN 978-1-4419-3196-2 978-1-4757-34843. URL http://link. springer.com/chapter/10.1007/978-1-4757-3484-3_ 17. DOI: $10.1007 / 978-1-4757-3484-3 \_17.11$

[139] Li-Qun Xu and Yongmin Li. Video classification using spatial-temporal features and PCA. In 2003 International Conference on Multimedia and Expo, 2003. ICME '03. Proceedings, volume 3, pages III-485-8 vol.3, July 2003. doi: 10.1109/ICME. 2003.1221354. 45 
[140] Clyde W. Yancy, Mariell Jessup, Biykem Bozkurt, Javed Butler, Donald E. Casey, Mark H. Drazner, Gregg C. Fonarow, Stephen A. Geraci, Tamara Horwich, James L. Januzzi, Maryl R. Johnson, Edward K. Kasper, Wayne C. Levy, Frederick A. Masoudi, Patrick E. McBride, John J. V. McMurray, Judith E. Mitchell, Pamela N. Peterson, Barbara Riegel, Flora Sam, Lynne W. Stevenson, W. H. Wilson Tang, Emily J. Tsai, and Bruce L. Wilkoff. 2013 ACCF/AHA Guideline for the Management of Heart Failure, Developed in Collaboration With the American College of Chest Physicians, Heart Rhythm Society and International Society for Heart and Lung Transplantation Endorsed by the American Association of Cardiovascular and Pulmonary Rehabilitation. Circulation, 128(16):e240-e327, October 2013. ISSN 0009-7322, 1524-4539. doi: 10.1161/CIR.0b013e31829e8776. URL http://circ.ahajournals.org/content/128/16/e240. 2

[141] C. Ye, M. T. Coimbra, and B. V. K. Vijaya Kumar. Arrhythmia detection and classification using morphological and dynamic features of ECG signals. In 2010 Annual International Conference of the IEEE Engineering in Medicine and Biology, pages 1918-1921, August 2010. doi: 10.1109/IEMBS.2010.5627645. 69

[142] Mohammed J Zaki, Wagner Meira Jr, and Wagner Meira. Data mining and analysis: fundamental concepts and algorithms. Cambridge University Press, 2014. 50

[143] Sheng Zhang and Matthew Turk. Eigenfaces. Scholarpedia, 3(9):4244, September 2008. ISSN 1941-6016. doi: 10.4249/scholarpedia.4244. URL http://www. scholarpedia.org/article/Eigenfaces. 45 


\section{Curriculum Vitae}

- Born 25.10.1985 in Hagen, NRW

- Abitur 2004 in at the Veit-Ludwig-von-Seckendorff Gymnasium in Meuselwitz, Thüringia

- Diploma in Physics 2013 at the Georg-August-Universität Göttingen

- Since 2013 enrolled in the PHD program PBCS at the Georg-August-Universität Göttingen 



\section{Acknowledgments}

I would like to thank my adviser Prof. Stefan Luther and Prof. Ulrich Parlitz for their unwavering support during the recent years.

The work presented in this document would have been impossible without the help of the members of the Biomedical Research Group at the Max Planck Institute for Dynamics and Self-Organization. Special thanks go to Dr. Daniel Hornung for his enormous patients during the preformed experiments as well as Dr. Alexander Schlemmer and Sebastian Berg for the provided expertise in data analysis. 